\title{
Pd-Catalyzed Stereoselective 1,2-Aryboration of
}

\author{
Alkenylarenes \\ Penglin Zhang, ${ }^{\mathrm{a}}$ Mimi Xing, ${ }^{\mathrm{a}}$ Qitao Guan, ${ }^{\mathrm{a}}$ Jinguo Zhang, ${ }^{\mathrm{a}}$ Qian Zhao ${ }^{\mathrm{a}}$ and Chun \\ Zhang *a, b
}

a. Tianjin Key Laboratory of Molecular Optoelectronic Science, Department of Chemistry, Institute of Molecular Plus, Tianjin University, Weijin Rd. 92, Tianjin 300072, China

b. State Key Laboratory of Elemento-Organic Chemistry Nankai University Tianjin 300071, China

E-mail: chunzhang@tju.edu.cn

\section{Supporting Information}

1. General information $\quad$ S3

2. Synthesis of substrates $\quad$ S4

3. The effect of different reaction conditions $\quad$ S5

$\begin{array}{ll}\text { 4. General procedure for the reaction } & \text { S7 }\end{array}$

5. Analytical data for compounds S8

$\begin{array}{ll}\text { 6. References } & \text { S21 }\end{array}$

$\begin{array}{ll}\text { 7. The data for some failed examples } & \text { S22 }\end{array}$

8. The data for $1 \mathrm{mmol}$ scale reaction $\quad \mathrm{S} 23$

$\begin{array}{lll}\text { 9. Result for X-ray analysis } & \text { S24 }\end{array}$

10. Determination of diastereoselectivity $\quad$ S25 
11. Identification of the absolute configuration

12. Enantiomeric ratios of products

13. NMR spectra of products

S32 


\section{General Considerations}

All manipulations were conducted with Schlenk tube. ${ }^{1} \mathrm{H}$ NMR spectra were recorded on Bruker AVIII-400 spectrometers. Chemical shifts (in ppm) were referenced to tetramethylsilane ( $\delta=0$ ppm) in $\mathrm{CDCl}_{3}$ as an internal standard. ${ }^{13} \mathrm{C}$ NMR spectra were obtained by using the same NMR spectrometers and were calibrated with $\mathrm{CDCl}_{3}(\delta=77.00 \mathrm{ppm})$. HPLC data were collected on a Shimadzu LC-2030 spectrometer. Optical rotations were determined using an Autopol IV automatic polarimeter. High resolution mass spectrometry (HRMS) data were obtained on a QTOF mass analyzer with electrospray ionization (ESI) through a Bruker Daltonicmior OTOF-QII. Substrates were purchased from Aldrich, TCI, Acros, Energy, Aladdin, or synthesized according to the procedures outlined below. Unless otherwise noted, materials obtained from commercial suppliers were used without further purification. 


\section{Synthesis of alkenes Substrates}

These substrates were prepared according to the corresponding literature reports. Analytical data $\left({ }^{1} \mathrm{H}\right.$ NMR, ${ }^{13} \mathrm{C}$ NMR) matches with the literature. All aryldiazonium salts were synthesized according to the literature. ${ }^{10}$<smiles>Fc1ccc2c(c1)C=CCC2</smiles>

1b: 6-fluoro-1,2-dihydronaphthalene ${ }^{1}$<smiles>Brc1ccc2c(c1)C=CCC2</smiles>

1d: 6-bromo-1,2-dihydronaphthalene ${ }^{1}$<smiles>C1=Cc2ccccc2CCC1</smiles>

1f: 6,7-dihydro-5H-benzo[7]annulene ${ }^{1}$<smiles>O=C(c1ccccc1)N1CC=Cc2ccccc21</smiles>

1i: benzyl quinoline-1 $(2 H)$-carboxylate ${ }^{3}$

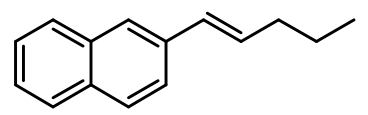

1k: $(E)-2-\left(\right.$ pent-1-en-1-yl)naphthalene ${ }^{5}$<smiles>C/C=C/c1ccc2cc(OC)ccc2c1</smiles>

1m: (E)-2-methoxy-6-(prop-1-en-1-yl)naphthalene ${ }^{7}$<smiles>C/C=C/c1ccc(C(C)=O)cc1</smiles>

1o: methyl (E)-4-(prop-1-en-1-yl) benzoate ${ }^{9}$<smiles>Clc1ccc2c(c1)C=CCC2</smiles>

1c: 6-chloro-1,2-dihydronaphthalene ${ }^{1}$<smiles>O=[N+]([O-])c1ccc2c(c1)C=CCC2</smiles>

1e:6-nitro-1,2-dihydronaphthalene ${ }^{1}$<smiles>C1=Cc2ccccc2OC1</smiles>

1h: $2 \mathrm{H}$-chromene ${ }^{2}$

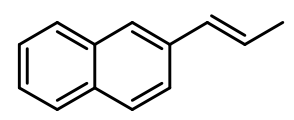

1j: (E)-2-(prop-1-en-1-yl)naphthalene ${ }^{4}$

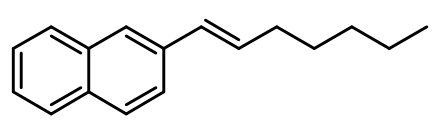

1I: (E)-2-(hept-1-en-1-yl)naphthalene ${ }^{6}$<smiles>C/C=C/c1ccc(Cl)cc1</smiles>

1n: (E)-1-chloro-4-(prop-1-en-1-yl)benzene ${ }^{8}$ 


\section{The effect of different reaction conditions}

Table S1. The effect of different bases and additives:

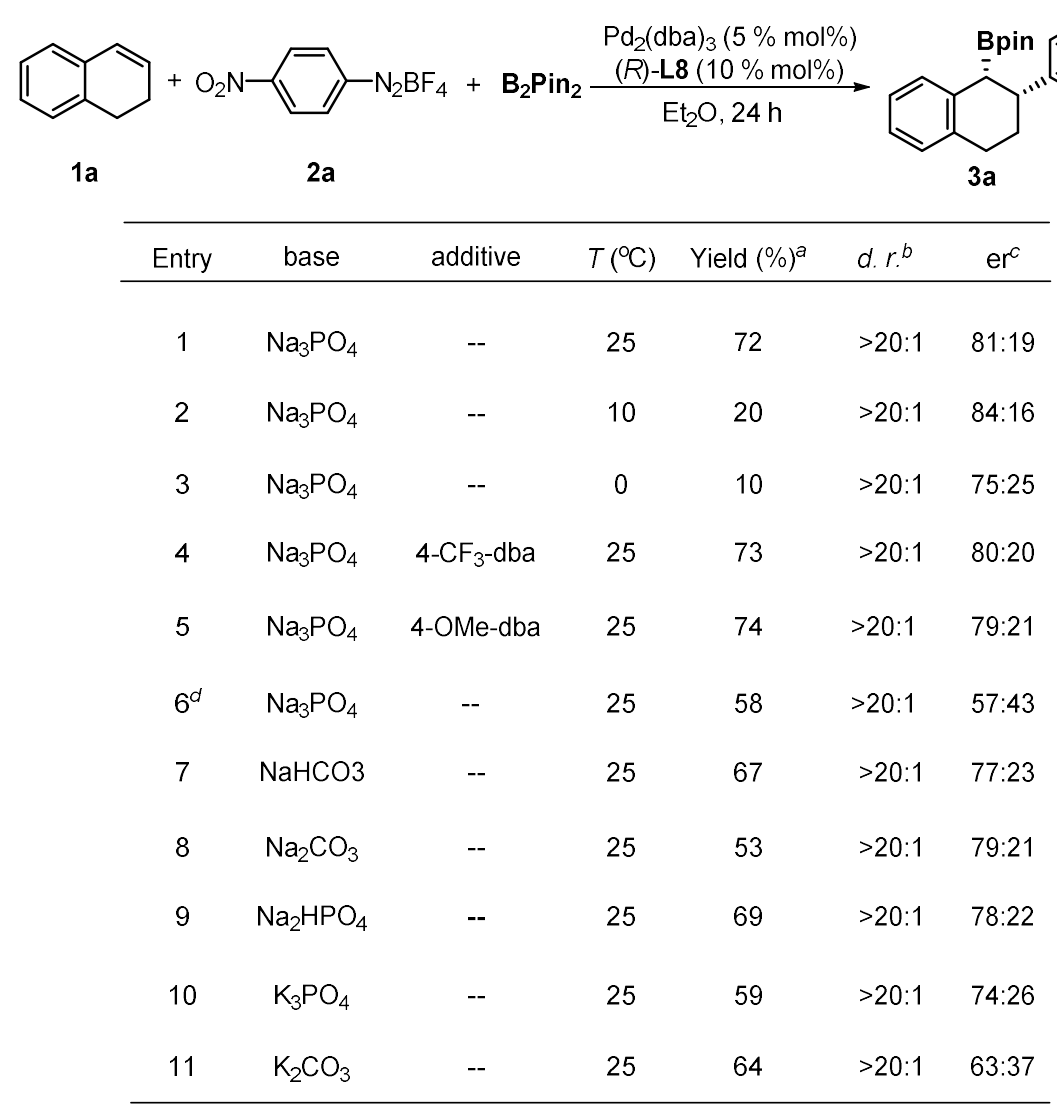

${ }^{a}$ Yield determined by ${ }^{1} \mathrm{H}$ NMR. ${ }^{b} d . r$ determined by ${ }^{1} \mathrm{H}$ NMR.${ }^{c}$ determined by chiral HPLC analysis. ${ }^{d} \mathrm{Pd}(\mathrm{OAc})_{2}$ instead of $\mathrm{Pd}_{2}(\mathrm{dba})_{3}$

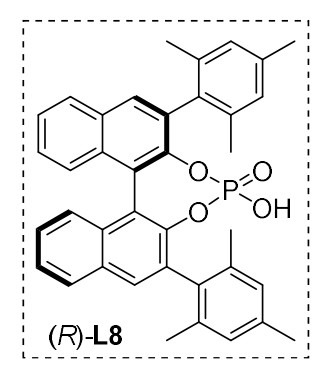


Table S2. The effect of different solvent and temperature:

\begin{tabular}{|c|c|c|c|c|c|}
\hline \multirow{2}{*}{$\frac{1 \mathrm{a}}{\text { Entry }}$} & \multicolumn{3}{|c|}{$\begin{array}{c}\mathrm{Pd}_{2}(\mathrm{dba})_{3}(5 \% \mathrm{~mol} \% \\
(R)-\mathrm{L} 8(10 \% \mathrm{~mol} \%) \\
\mathrm{Na}_{3} \mathrm{PO}_{4}(2.0 \mathrm{eq}) \\
24 \mathrm{~h}\end{array}$} & \multicolumn{2}{|c|}{$3 a$} \\
\hline & Sovent & $T\left({ }^{\circ} \mathrm{C}\right)$ & Yield $(\%)^{a}$ & d. $r^{b}$ & $e r^{c}$ \\
\hline 1 & $\mathrm{Et}_{2} \mathrm{O}$ & 25 & 72 & $>20: 1$ & $81: 19$ \\
\hline 2 & $\mathrm{n}$ - hexene & 25 & 11 & $>20: 1$ & $65: 35$ \\
\hline 3 & MTBE & 25 & 78 & $>20: 1$ & $77: 23$ \\
\hline 4 & toluene & 25 & 67 & $>20: 1$ & $54: 46$ \\
\hline 5 & THF & 25 & 67 & $>20: 1$ & $67: 33$ \\
\hline 6 & toluene:MTBE = $3: 2$ & 25 & 76 & $>20: 1$ & $63: 37$ \\
\hline 7 & toluene: $\mathrm{MTBE}=3: 2$ & 10 & 62 & $>20: 1$ & $83: 17$ \\
\hline 8 & toluene: $\mathrm{Et}_{2} \mathrm{O}=3: 2$ & 10 & 48 & $>20: 1$ & $70: 30$ \\
\hline 9 & toluene:MTBE = 3:2 & 0 & 43 & $>20: 1$ & $87: 13$ \\
\hline 10 & toluene:MTBE = 1:1 & 0 & 27 & $>20: 1$ & $83: 17$ \\
\hline 11 & toluene:MTBE = 2:3 & 0 & 20 & $>20: 1$ & $79: 21$ \\
\hline 12 & benzene:MTBE = 3:2 & 0 & 82 & $>20: 1$ & $84: 16$ \\
\hline 13 & Chlorobenzene:MTBE = 3:2 & 0 & 73 & $>20: 1$ & $78: 22$ \\
\hline 14 & HexafluoroBenzene:MTBE = 3:2 & 0 & 13 & $>20: 1$ & $79: 21$ \\
\hline 15 & dimethylbenzene:MTBE = 3:2 & 0 & 20 & $>20: 1$ & $82: 18$ \\
\hline 16 & Mesitylene:MTBE = 1:1 & 0 & 13 & $>20: 1$ & $79: 21$ \\
\hline $17^{d}$ & benzene: $\mathrm{MTBE}=3: 2$ & 0 & 50 & $>20: 1$ & $89: 11$ \\
\hline $18^{d}$ & toluene: $\mathrm{MTBE}=3: 2$ & 0 & 20 & $>20: 1$ & $93: 7$ \\
\hline
\end{tabular}

a Yield determined by ${ }^{1} \mathrm{H}$ NMR of the corresponding alcohol product. ${ }^{b}$ d.r. determined by ${ }^{1} \mathrm{H}$ NMR of of the corresponding alcohol product. ${ }^{c}$ determined by chiral HPLC analysis of the corresponding alcohol product. ${ }^{d}(R)$-L12 instead of $(R)$-L8.
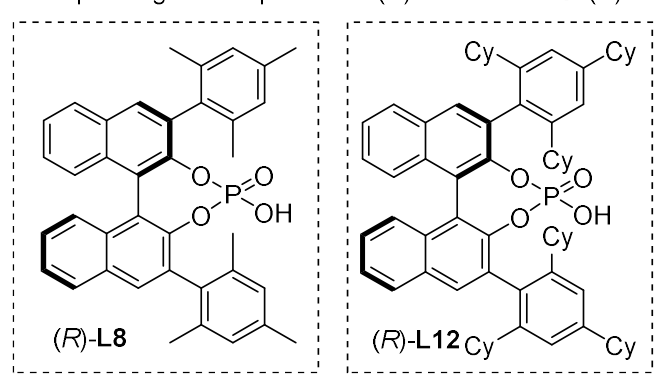


\section{General procedure for the reaction}

General procedure A:

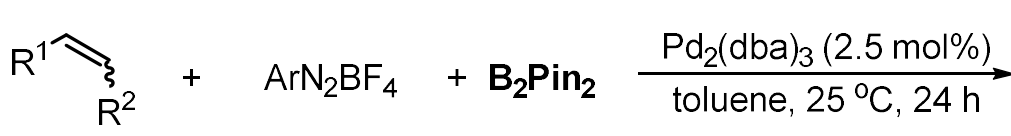
1

2<smiles>[R]C([Al])C([R])Br</smiles>

3

In an oven dried 25-ml Schlenk tube containing a stirring bar was charged with charged with $\mathrm{B}_{2} \mathrm{Pin}_{2}(0.3 \mathrm{mmol}), \mathrm{Pd}_{2} \mathrm{dba}_{3}(2.5 \mathrm{~mol} \%)$, and $\mathrm{ArN}_{2} \mathrm{BF}_{4}(0.3 \mathrm{mmol})$. The tube was then evacuated and back-filled under a $\mathrm{N}_{2}$ flow (this sequence was repeated three times). Anhydrous toluene (2.0 $\mathrm{ml})$, alkene $1(0.20 \mathrm{mmol})$ were added subsequently under $\mathrm{N}_{2}$. The tube was stirred at $25^{\circ} \mathrm{C}$ for $24 \mathrm{~h}$. The reaction was concentrated by rotary evaporation. The residue was purified by silica gel column chromatography (EtOAc/petroleum ether) to afford the product 3.

General procedure B:

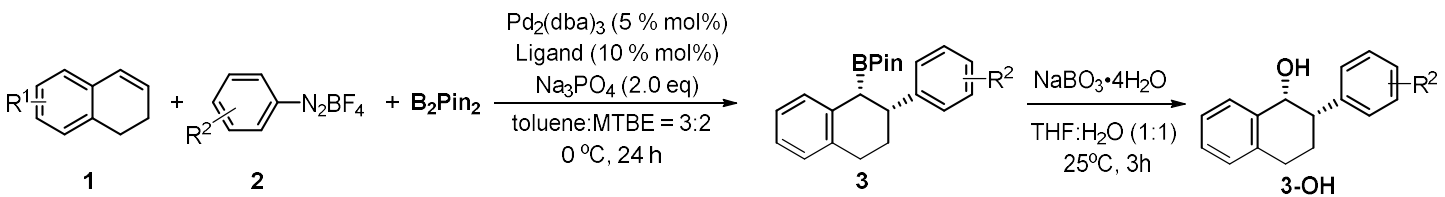

In an oven dried 10-ml Schlenk tube containing a stirring bar was charged with charged with $\mathrm{B}_{2} \mathrm{Pin}_{2}(0.20 \mathrm{mmol}), \mathrm{Pd}_{2} \mathrm{dba}_{3}(5 \mathrm{~mol} \%), \mathbf{L}(10 \mathrm{~mol} \%), \mathrm{Na}_{3} \mathrm{PO}_{4}(0.20 \mathrm{mmol})$, the $\mathrm{ArN}_{2} \mathrm{BF}_{4}(0.20$ $\mathrm{mmol}$ ). The tube was then evacuated and back-filled under a $\mathrm{N}_{2}$ flow (this sequence was repeated three times). Indicate solvent $(1.0 \mathrm{ml})$ was added under $\mathrm{N}_{2}$. The reaction mixture was stirred for 10 min at $\mathrm{rt}$ and then alkene $1(0.10 \mathrm{mmol})$ was added, the tube was stirred at $0{ }^{\circ} \mathrm{C}$ for $24 \mathrm{~h}$. the reaction was filtered through a plug of cotton and eluted with EtOAc $(3 \times 2 \mathrm{~mL})$. The filtrate was concentrated by rotary evaporation to provide yellow oil, and then dissolved in tetrahydrofuran (THF, $1.0 \mathrm{~mL}) . \mathrm{NaBO}_{3} \cdot 4 \mathrm{H}_{2} \mathrm{O}\left(76.9 \mathrm{mg}, 0.500 \mathrm{mmol}\right.$,) and $\mathrm{H}_{2} \mathrm{O}(1.0 \mathrm{~mL})$ were added. The resulting mixture was allowed to stir at $25{ }^{\circ} \mathrm{C}$ for three hours. The reaction mixture diluted with EtOAc $(5.0 \mathrm{ml})$ and $\mathrm{H}_{2} \mathrm{O}(5.0 \mathrm{ml})$. Then it was extracted with EtOAc $(5.0 \mathrm{ml} \times 3)$. The organic layer was combined and dried over $\mathrm{Na}_{2} \mathrm{SO}_{4}$. Then filtered and concentrated by rotary evaporation. The residue was purified by silica gel chromatography (EtOAc/petroleum ether) to afford the product 3-OH. 


\section{Analytical data for compounds}<smiles>CC1(C)OB(C2c3ccccc3CCC2c2ccc([N+](=O)[O-])cc2)OC1(C)C</smiles>

\section{4,4,5,5-tetramethyl-2-(2-(4-nitrophenyl)-1,2,3,4-tetrahydronaphthalen-1-yl)-1,3,2-dioxaborol}

ane (3a): The general procedure A was followed using 1,2-dihydronaphthalene (1a, $26 \mathrm{mg}, 0.20$ $\mathrm{mmol}$ ) and 4-nitrophenyldiazonium tetrafluoroborate $(\mathbf{2 a}, 71 \mathrm{mg}, 0.30 \mathrm{mmol})$. Purification of this material by chromatography on silica gel (EtOAc : petroleum ether $=1: 20$ ) afforded product 3a as a colorless solid $(55 \mathrm{mg}, 73 \%$ yield, $>20: 1 \mathrm{dr}): \mathrm{Rf}=0.6($ EtOAc $:$ petroleum ether $=1: 10) ;{ }^{1} \mathbf{H}$ NMR $\left(\mathrm{CDCl}_{3}, 400 \mathrm{MHz}\right) \delta: 8.16(\mathrm{~d}, J=8.0 \mathrm{~Hz}, 2 \mathrm{H}), 7.51(\mathrm{~d}, J=8.0 \mathrm{~Hz}, 2 \mathrm{H}), 7.15-7.08(\mathrm{~m}, 4 \mathrm{H})$, 3.32-3.28 (m, 1H), 3.05-2.91 (m, 3H), 2.59-2.49 (m, 1H), 2.16-2.13 (m, 1H), $0.96(\mathrm{~s}, 6 \mathrm{H}), 0.92(\mathrm{~s}$, $6 \mathrm{H}) ;{ }^{13} \mathbf{C}$ NMR $\left(\mathrm{CDCl}_{3}, 100 \mathrm{MHz}\right) \delta: 153.6,146.3,136.7,135.6,129.4,128.6,128.5,125.5$, 125.3, $123.3,83.2,41.3,29.3,25.2,24.6,24.1 \mathrm{ppm}$; HRMS (ESI-TOF) $m / z$ calcd for $\mathrm{C}_{22} \mathrm{H}_{27} \mathrm{BO}_{4} \mathrm{~N}(\mathrm{M}+$ $\mathrm{H})^{+}: 379.2069$, found 379.2073 .

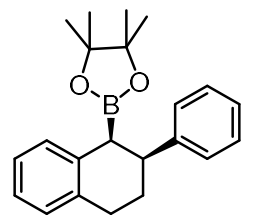

$3 \mathrm{~b}$

4,4,5,5-tetramethyl-2-(2-phenyl-1,2,3,4-tetrahydronaphthalen-1-yl)-1,3,2-dioxaborolane (3b): The general procedure A was followed using 1,2-dihydronaphthalene (1a, $26 \mathrm{mg}, 0.20 \mathrm{mmol})$ and phenyldiazonium tetrafluoroborate $(\mathbf{2} \mathbf{b}, 58 \mathrm{mg}, 0.30 \mathrm{mmol})$. Purification of this material by chromatography on silica gel (EtOAc : petroleum ether $=1: 40$ ) afforded product $\mathbf{3 b}$ as a colorless solid $(56 \mathrm{mg}, 84 \%$ yield, $>20: 1 \mathrm{dr}): \mathrm{Rf}=0.8($ EtOAc : petroleum ether $=1: 10) ;{ }^{1} \mathbf{H}$ NMR $\left(\mathrm{CDCl}_{3}, 400 \mathrm{MHz}\right) \delta: 7.35-7.33(\mathrm{~m}, 2 \mathrm{H}), 7.29-7.23(\mathrm{~m}, 2 \mathrm{H}), 7.19-7.07(\mathrm{~m}, 5 \mathrm{H}), 3.21-3.19$ (m, 1H), 3.06-2.89 (m, 3H), 2.55-2.45 (m, 1H), 2.14-2.11 (m, 1H), $0.97(\mathrm{~s}, 6 \mathrm{H}), 0.88(\mathrm{~s}, 6 \mathrm{H}) ;{ }^{13} \mathrm{C}$ NMR $\left(\mathrm{CDCl}_{3}, 100 \mathrm{MHz}\right) \delta: 145.6,137.7,136.1,129.4,128.6,128.0,127.6,125.9,125.3,124.9$, 82.9, 41.0, 29.7, 25.3, 24.5, $24.2 \mathrm{ppm}$; HRMS (ESI-TOF) $\mathrm{m} / z$ calcd for $\mathrm{C}_{22} \mathrm{H}_{28} \mathrm{BO}_{2}(\mathrm{M}+\mathrm{H})^{+}$: 334.2219, found 334.2220.

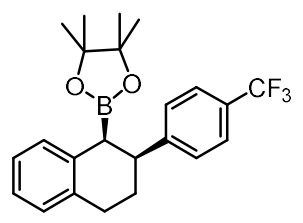

$3 c$

4,4,5,5-tetramethyl-2-(2-(4-(trifluoromethyl)phenyl)-1,2,3,4-tetrahydronaphthalen-1-yl)-1,3,2 -dioxaborolane (3c): The general procedure A was followed using 1,2-dihydronaphthalene (1a, 26 $\mathrm{mg}, 0.20 \mathrm{mmol}$ ) and 4-(trifluoromethyl)phenyl diazonium tetrafluoroborate $(\mathbf{2 c}, 78 \mathrm{mg}, 0.30$ mmol). Purification of this material by chromatography on silica gel (EtOAc : petroleum ether $=$ $1: 40$ ) afforded product $3 \mathbf{c}$ as a colorless solid $(73 \mathrm{mg}, 91 \%$ yield, $>20: 1 \mathrm{dr}$ ): $\mathrm{Rf}=0.8$ (EtOAc : petroleum ether =1: 10); ${ }^{1} \mathbf{H}$ NMR $\left(\mathrm{CDCl}_{3}, 400 \mathrm{MHz}\right) \delta: 7.55(\mathrm{~d}, J=8.0 \mathrm{~Hz}, 2 \mathrm{H}), 7.46(\mathrm{~d}, J=$ $8.0 \mathrm{~Hz}, 2 \mathrm{H}), 7.14-7.07(\mathrm{~m}, 4 \mathrm{H}), 3.29-3.24(\mathrm{~m}, 1 \mathrm{H}), 3.05-2.90(\mathrm{~m}, 3 \mathrm{H}), 2.56-2.46(\mathrm{~m}, 1 \mathrm{H})$, 2.15-2.12 (m, 1H), $0.96(\mathrm{~s}, 6 \mathrm{H}), 0.89(\mathrm{~s}, 6 \mathrm{H}) ;{ }^{13} \mathbf{C} \mathbf{~ N M R}\left(\mathrm{CDCl}_{3}, 100 \mathrm{MHz}\right) \delta: 149.8,137.1,135.8$, 
129.4, 128.6, 128.0, 125.5, 125.1, 124.97, 124.93, 124.89, 124.85, 83.1, 41.0, 29.5, 25.2, 24.4, $24.1 \mathrm{ppm}$; HRMS (ESI-TOF) $\mathrm{m} / z$ calcd for $\mathrm{C}_{23} \mathrm{H}_{27} \mathrm{BO}_{2} \mathrm{~F}_{3}(\mathrm{M}+\mathrm{H})^{+}: 403.2060$, found 403.2061.

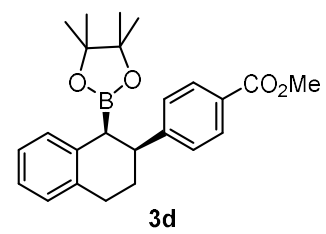

Methyl4-(1-(4,4,5,5-tetramethyl-1,3,2-dioxaborolan-2-yl)-1,2,3,4-tetrahydronaphthalen-2-yl) benzoate (3d): The general procedure A was followed using 1,2-dihydronaphthalene (1aa, $26 \mathrm{mg}$, $0.20 \mathrm{mmol})$ and The correspondinge diazonium tetrafluoroborate $(\mathbf{2 d}, 75 \mathrm{mg}, 0.30 \mathrm{mmol})$. Purification of this material by chromatography on silica gel (EtOAc : petroleum ether $=1: 10)$ afforded product 3d as a yellow solid ( $66 \mathrm{mg}, 85 \%$ yield, $>20: 1 \mathrm{dr}$ ): $\mathrm{Rf}=0.2$ (EtOAc : petroleum ether = $1: 10)$; ${ }^{1} \mathbf{H}$ NMR $\left(\mathrm{CDCl}_{3}, 400 \mathrm{MHz}\right) \delta: 7.97(\mathrm{~d}, J=7.9 \mathrm{~Hz}, 2 \mathrm{H}), 7.42(\mathrm{~d}, J=7.9 \mathrm{~Hz}, 2 \mathrm{H})$, 7.13-7.07 (m, 4H), $3.91(\mathrm{~s} 3 \mathrm{H}), 3.27-3.23(\mathrm{~m}, 1 \mathrm{H}), 3.05-2.90(\mathrm{~m}, 3 \mathrm{H}), 2.58-2.48(\mathrm{~m}, 1 \mathrm{H})$, 2.14-2.11 (m, 1H), $0.96(\mathrm{~s}, 6 \mathrm{H}), 0.90(\mathrm{~s}, 6 \mathrm{H}) ;{ }^{13} \mathbf{C} \mathbf{~ N M R}\left(\mathrm{CDCl}_{3}, 100 \mathrm{MHz}\right) \delta: 167.2,151.2,137.2$, 135.8, 129.4, 128.6, 127.8, 127.7, 125.4, 125.0, 83.0, 52.0, 41.2, 29.5, 25.2, 24.5, $24.2 \mathrm{ppm;}$ HRMS (ESI-TOF) $\mathrm{m} / z$ calcd for $\mathrm{C}_{24} \mathrm{H}_{30} \mathrm{BO}_{4}(\mathrm{M}+\mathrm{H})^{+}: 393.2242$, found 393.2237.<smiles>CC(C)(C)O[C@H](c1cc(F)cc(F)c1)C1CCc2ccccc2O1</smiles>

2-(2-(3,5-difluorophenyl)-1,2,3,4-tetrahydronaphthalen-1-yl)-4,4,5,5-tetramethyl-1,3,2-dioxa borolane (3e): The general procedure A was followed using 1,2-dihydronaphthalene (1a, $26 \mathrm{mg}$, $0.20 \mathrm{mmol}$ ) and 3,5-difluorophenyl diazonium tetrafluoroborate (2e, $68 \mathrm{mg}, 0.30 \mathrm{mmol})$. Purification of this material by chromatography on silica gel (EtOAc : petroleum ether $=1: 40$ ) afforded product $3 \mathbf{e}$ as a yellow solid $(61 \mathrm{mg}, 83 \%$ yield, $>20: 1 \mathrm{dr}$ ): $\mathrm{Rf}=0.8$ (EtOAc : petroleum ether = 1: 10); ${ }^{1} \mathbf{H}$ NMR $\left(\mathrm{CDCl}_{3}, 400 \mathrm{MHz}\right) \delta: 7.11-7.03(\mathrm{~m}, 4 \mathrm{H}), 6.89-6.87(\mathrm{~m}, 2 \mathrm{H}), 6.65-6.61$ (m, 1H), 3.16-3.13 (m, 1H), 3.00-2.89 (m, 3H), 2.53-2.43 (m, 1H), 2.08-2.04 (m, 1H), $1.01(\mathrm{~s}, 6 \mathrm{H})$, $0.96(\mathrm{~s}, 6 \mathrm{H}) ;{ }^{13} \mathrm{C}$ NMR $\left(\mathrm{CDCl}_{3}, 100 \mathrm{MHz}\right) \delta: 164.2,164.0,161.7,161.6,150.1,150.0,149.9$, 136.9, 135.6, 129.4, 128.6, 125.5, 125.2, 110.63, 110.57, 110.45, 110.39, 101.5, 101.3, 101.0, 83.1, 41.2, 29.5, 25.2, 24.5, $24.2 \mathrm{ppm}$; HRMS (ESI-TOF) $\mathrm{m} / \mathrm{z}$ calcd for $\mathrm{C}_{22} \mathrm{H}_{26} \mathrm{BO}_{2} \mathrm{~F}_{2}(\mathrm{M}+\mathrm{H})^{+}$: 370.2030 , found 370.2019 .<smiles>CCC(C(=O)OC(C)(C)C)c1ccc(F)cc1</smiles>

2-(2-(4-fluorophenyl)-1,2,3,4-tetrahydronaphthalen-1-yl)-4,4,5,5-tetramethyl-1,3,2-dioxaboro lane (3f): The general procedure A was followed using 1,2-dihydronaphthalene (1a, $26 \mathrm{mg}, 0.20$ $\mathrm{mmol}$ ) and 3,5-difluorophenyl diazonium tetrafluoroborate (2f, $63 \mathrm{mg}, 0.30 \mathrm{mmol})$. Purification of this material by chromatography on silica gel (EtOAc : petroleum ether $=1: 40$ ) afforded product 3f as a colorless solid ( $63 \mathrm{mg}, 90 \%$ yield, $>20: 1 \mathrm{dr}): \mathrm{Rf}=0.8($ EtOAc $:$ petroleum ether $=1: 10)$; ${ }^{1} \mathbf{H}$ NMR $\left(\mathrm{CDCl}_{3}, 400 \mathrm{MHz}\right)$ 8: 7.31-7.28 (m, 2H), 7.13-7.06 (m, 4H), 7.00-6.95 (m, 2H), 
3.20-3.16 (m, 1H), 3.03-2.89 (m, 3H), 2.51-2.41 (m, 1H), 2.11-2.08 (m, 1H), $0.98(\mathrm{~s} 6 \mathrm{H}), 0.91(\mathrm{~s}$, $6 \mathrm{H}) ;{ }^{13} \mathbf{C}$ NMR $\left(\mathrm{CDCl}_{3}, 100 \mathrm{MHz}\right) \delta: 162.5,160.1,141.33,141.30,137.4,135.9,129.4,129.0$, 128.9, 128.6, 125.4, 125.0, 114.8, 114.6, 83.0, 40.3, 29.6, 25.5, 24.5, 24.2 ppm; HRMS (ESI-TOF) $\mathrm{m} / \mathrm{z}$ calcd for $\mathrm{C}_{22} \mathrm{H}_{27} \mathrm{BO}_{2} \mathrm{~F}(\mathrm{M}+\mathrm{H})^{+}: 352.2124$, found 352.2120 .

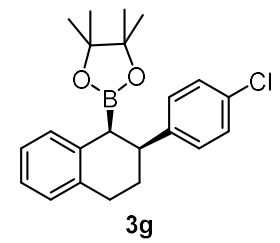

2-(2-(4-chlorophenyl)-1,2,3,4-tetrahydronaphthalen-1-yl)-4,4,5,5-tetramethyl-1,3,2-dioxabor olane (3g): The general procedure A was followed using 1,2-dihydronaphthalene (1a, $26 \mathrm{mg}, 0.20$ $\mathrm{mmol})$ and 4-chlorophenyl diazonium tetrafluoroborate $(2 \mathrm{~g}, 68 \mathrm{mg}, 0.30 \mathrm{mmol})$. Purification of this material by chromatography on silica gel (EtOAc : petroleum ether $=1: 40$ ) afforded product $3 \mathrm{~g}$ as a colorless solid $(69 \mathrm{mg}, 94 \%$ yield, $>20: 1 \mathrm{dr}): \mathrm{Rf}=0.8$ (EtOAc $:$ petroleum ether $=1: 10)$; ${ }^{1} \mathbf{H}$ NMR $\left(\mathrm{CDCl}_{3}, 400 \mathrm{MHz}\right)$ 8: 7.29-7.24 (m, 4H), 7.11-7.06 (m, 4H), 3.19-3.15 (m, $\left.1 \mathrm{H}\right)$, 3.02-2.88 (m, 3H), 2.51-2.41 (m, 1H), 2.10-2.07 (m, 1H), $0.98(\mathrm{~s}, 6 \mathrm{H}), 0.92(\mathrm{~s}, 6 \mathrm{H}) ;{ }^{13} \mathbf{C}$ NMR $\left(\mathrm{CDCl}_{3}, 100 \mathrm{MHz}\right) \delta: 144.1,137.3,135.8,131.6,129.4,129.0,128.6,128.0,125.4,125.0,83.0$, 40.4, 29.5, 25.3, 24.5, $24.2 \mathrm{ppm}$; HRMS (ESI-TOF) $\mathrm{m} / \mathrm{z}$ calcd for $\mathrm{C}_{22} \mathrm{H}_{27} \mathrm{BO}_{2} \mathrm{Cl}(\mathrm{M}+\mathrm{H})^{+}$: 369.1797, found 369.1798 .

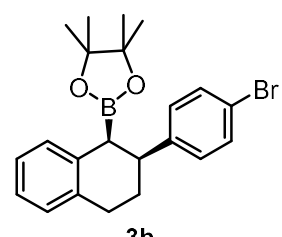

$3 \mathrm{~h}$

2-(2-(4-bromophenyl)-1,2,3,4-tetrahydronaphthalen-1-yl)-4,4,5,5-tetramethyl-1,3,2-dioxabor olane (3h): The general procedure A was followed using 1,2-dihydronaphthalene (1a, $26 \mathrm{mg}, 0.20$ $\mathrm{mmol}$ ) and 4-bromophenyl diazonium tetrafluoroborate $(\mathbf{2} \mathbf{h}, 81 \mathrm{mg}, 0.30 \mathrm{mmol})$. Purification of this material by chromatography on silica gel (EtOAc : petroleum ether $=1: 40$ ) afforded product $\mathbf{3 h}$ as a yellow solid $(72 \mathrm{mg}, 87 \%$ yield, $>20: 1 \mathrm{dr}): \mathrm{Rf}=0.8$ (EtOAc $:$ petroleum ether $=1: 10) ;{ }^{1} \mathbf{H}$ NMR $\left(\mathrm{CDCl}_{3}, 400 \mathrm{MHz}\right) \delta: 7.41(\mathrm{~d}, J=7.8 \mathrm{~Hz}, 2 \mathrm{H}), 7.22(\mathrm{~d}, J=7.8 \mathrm{~Hz}, 2 \mathrm{H}), 7.12-7.07$ (m, 4H), 3.18-3.14 (m, 1H), 3.01-2.88 (m, 3H), 2.51-2.41 (m, 1H), 2.09-2.06 (m, 1H), $0.98(\mathrm{~s}, 6 \mathrm{H}), 0.92(\mathrm{~s}$, $6 \mathrm{H}) ;{ }^{13} \mathrm{C}$ NMR $\left(\mathrm{CDCl}_{3}, 100 \mathrm{MHz}\right) \delta: 144.7,137.3,135.9,131.0,129.43,129.37,128.6,125.4$, 125.0, 119.7, 83.0, 40.5, 29.5, 25.3, 24.5, $24.2 \mathrm{ppm}$; HRMS (ESI-TOF) $\mathrm{m} / \mathrm{z}$ calcd for $\mathrm{C}_{22} \mathrm{H}_{27} \mathrm{BO}_{2} \mathrm{Br}(\mathrm{M}+\mathrm{H})^{+}:$412.1324, found 412.1321 .

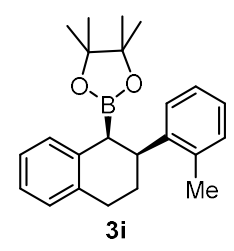

4,4,5,5-tetramethyl-2-(2-(o-tolyl)-1,2,3,4-tetrahydronaphthalen-1-yl)-1,3,2-dioxaborolane (3i): The general procedure A was followed using 1,2-dihydronaphthalene (1a, $26 \mathrm{mg}, 0.20 \mathrm{mmol})$ and $o$-tolyldiazonium tetrafluoroborate $(2 \mathbf{i}, 62 \mathrm{mg}, 0.30 \mathrm{mmol})$. Purification of this material by chromatography on silica gel (EtOAc : petroleum ether $=1: 40$ ) afforded product $\mathbf{3 i}$ as a colorless liquid $(42 \mathrm{mg}, 61 \%$ yield, $>20: 1 \mathrm{dr}): \mathrm{Rf}=0.8($ EtOAc : petroleum ether $=1: 10) ;{ }^{1} \mathbf{H}$ NMR 
$\left(\mathrm{CDCl}_{3}, 400 \mathrm{MHz}\right)$ 8: 7.36-7.35 (m, 1H), 7.11-7.08 (m, 7H), 3.35-3.32 (m, 1H), 3.02-2.90 (m, 3H), 2.66-2.56 (m, 1H), 2.36 (s 3H), 2.00-1.97 (m, 1H), 1.03 (s, 6H), $0.90(\mathrm{~s}, 6 \mathrm{H}) ;{ }^{13} \mathbf{C}$ NMR $\left(\mathrm{CDCl}_{3}\right.$, $100 \mathrm{MHz}) \delta: 143.7,138.0,136.2,135.9,130.1,129.4,128.7,126.9,125.8,125.7,125.3,124.9$, 82.9, 37.9, 30.0, 26.0, 24.5, 24.4, 19.4 ppm; HRMS (ESI-TOF) $\mathrm{m} / \mathrm{z}$ calcd for $\mathrm{C}_{23} \mathrm{H}_{30} \mathrm{BO}_{2}(\mathrm{M}+\mathrm{H})^{+}$: 348.2375 , found 348.2369 .<smiles>Cc1cccc(C2CCc3ccccc3P2C2OC(C)(C)C(C)(C)O2)c1</smiles>

$3 \mathbf{j}$

\section{4,4,5,5-tetramethyl-2-(2-(m-tolyl)-1,2,3,4-tetrahydronaphthalen-1-yl)-1,3,2-dioxaborolane}

(3j): The general procedure A was followed using 1,2-dihydronaphthalene (1a, $26 \mathrm{mg}, 0.20 \mathrm{mmol})$ and $m$-tolyldiazonium tetrafluoroborate $(\mathbf{2 j}, 62 \mathrm{mg}, 0.30 \mathrm{mmol})$. Purification of this material by chromatography on silica gel (EtOAc : petroleum ether $=1: 40$ ) afforded product $\mathbf{3 j a s}$ a colorless liquid $(54 \mathrm{mg}, 78 \%$ yield, $>20: 1 \mathrm{dr}): \mathrm{Rf}=0.8($ EtOAc : petroleum ether $=1: 10) ;{ }^{1} \mathbf{H}$ NMR $\left(\mathrm{CDCl}_{3}, 400 \mathrm{MHz}\right) \delta:$ 7.17-7.06 (m, 7H), 6.99-6.98 (m, 1H), 3.16-3.14 (m, 1H), 3.03-2.89 (m, 3H), 2.56-2.46 (m, 1H), $2.32(\mathrm{~s}, 3 \mathrm{H}), 2.11-2.05(\mathrm{~m}, 1 \mathrm{H}), 0.98(\mathrm{~s}, 6 \mathrm{H}), 0.90(\mathrm{~s}, 6 \mathrm{H}) ;{ }^{13} \mathbf{C} \mathbf{~ N M R}\left(\mathrm{CDCl}_{3}\right.$, $100 \mathrm{MHz}) \delta$ : 145.6, 137.8, 137.3, 136.1, 129.4, 128.6, 128.2, 128.0, 126.6, 125.3, 124.9, 124.7, 82.8, 41.1, 29.9, 25.4, 24.5, 24.2, 21.5 ppm; HRMS (ESI-TOF) $\mathrm{m} / z$ calcd for $\mathrm{C}_{23} \mathrm{H}_{30} \mathrm{BO}_{2}(\mathrm{M}+\mathrm{H})^{+}$: 348.2375 , found 348.2380 .

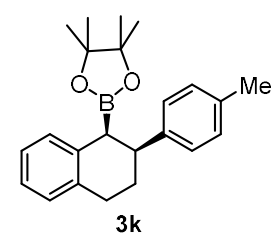

\section{4,4,5,5-tetramethyl-2-(2-(p-tolyl)-1,2,3,4-tetrahydronaphthalen-1-yl)-1,3,2-dioxaborolane}

(3k): The general procedure A was followed using 1,2-dihydronaphthalene (1a, $26 \mathrm{mg}, 0.20 \mathrm{mmol}$ ) and $p$-tolyldiazonium tetrafluoroborate $(\mathbf{2 k}, 62 \mathrm{mg}, 0.30 \mathrm{mmol})$. Purification of this material by chromatography on silica gel (EtOAc : petroleum ether $=1: 40$ ) afforded product $\mathbf{3 k}$ as a colorless solid (53 mg, 76\% yield, $>20: 1 \mathrm{dr}): \mathrm{Rf}=0.8($ EtOAc : petroleum ether $=1: 10) ;{ }^{1} \mathbf{H}$ NMR $\left(\mathrm{CDCl}_{3}, 400 \mathrm{MHz}\right) \delta$ : 7.23-7.21 (m, 2H), 7.10-7.07 (m, 6H), 3.17-3.14 (m, 1H), 3.03-2.88 (m, 3H), 2.53-2.43 (m, 1H), 2.31 (s 3H), 2.11-2.08 (m, 1H), 0.99 (s, 6H), 0.90 (s, 6H); ${ }^{13} \mathbf{C}$ NMR $\left(\mathrm{CDCl}_{3}, 100 \mathrm{MHz}\right) \delta: 142.6,137.9,136.2,135.3,129.3,128.7,128.6,127.4,125.3,124.9,82.9$, 40.7, 29.8, 25.6, 24.5, 24.2, 21.0 ppm; HRMS (ESI-TOF) $\mathrm{m} / z$ calcd for $\mathrm{C}_{23} \mathrm{H}_{30} \mathrm{BO}_{2}(\mathrm{M}+\mathrm{H})^{+}$: 348.2375 , found 348.2365 .<smiles>CC(C)(C)OPC1c2ccccc2CCC1c1ccc(Br)cc1</smiles>

31

2-(2-(4-(tert-butyl)phenyl)-1,2,3,4-tetrahydronaphthalen-1-yl)-4,4,5,5-tetramethyl-1,3,2-dioxa borolane (3I): The general procedure A was followed using 1,2-dihydronaphthalene (1a, $26 \mathrm{mg}$, $0.20 \mathrm{mmol}$ ) and 4-(tert-butyl)phenyl diazonium tetrafluoroborate $(21,74 \mathrm{mg}, 0.30 \mathrm{mmol})$. Purification of this material by chromatography on silica gel (EtOAc : petroleum ether $=1: 40$ ) 
afforded product $\mathbf{3 l}$ as a yellow liquid $(74 \mathrm{mg}, 95 \%$ yield, $>20: 1 \mathrm{dr}$ ): $\mathrm{Rf}=0.8$ (EtOAc : petroleum ether = 1: 10); ${ }^{1} \mathbf{H}$ NMR $\left(\mathrm{CDCl}_{3}, 400 \mathrm{MHz}\right) \delta: 7.33-7.26(\mathrm{~m}, 4 \mathrm{H}), 7.13-7.12(\mathrm{~m}, 1 \mathrm{H}), 7.07-7.05$ (m, 3H), 3.22-3.18 (m, 1H), 3.07-2.89 (m, 3H), 2.51-2.41 (m, 1H), 2.16-2.13 (m, 1H), 1.29 (s, 9H), 0.96 (s, 6H), 0.84 (s 6,H); ${ }^{13} \mathbf{C}$ NMR $\left(\mathrm{CDCl}_{3}, 100 \mathrm{MHz}\right) \delta$ : 148.8, 142.5, 137.8, 136.1, 129.4, 128.6, 127.1, 125.3, 124.9, 124.8, 82.8, 40.3, 34.3, 31.4, 29.8, 25.2, 24.34, 24.30 ppm; HRMS (ESI-TOF) $\mathrm{m} / \mathrm{z}$ calcd for $\mathrm{C}_{26} \mathrm{H}_{34} \mathrm{BO}_{2}(\mathrm{M}-\mathrm{H})^{-}: 388.2688$, found 388.2694 .

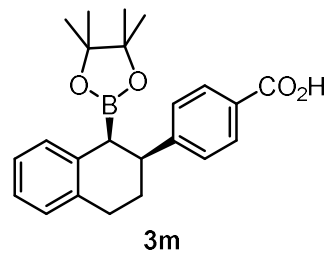

4-(1-(4,4,5,5-tetramethyl-1,3,2-dioxaborolan-2-yl)-1,2,3,4-tetrahydronaphthalen-2-yl)benzoic acid (3m): The general procedure A was followed using 1,2-dihydronaphthalene (1a, $26 \mathrm{mg}, 0.20$ $\mathrm{mmol})$ and The correspondinge diazonium tetrafluoroborate $(\mathbf{2 m}, 71 \mathrm{mg}, 0.30 \mathrm{mmol})$. Purification of this material by chromatography on silica gel (EtOAc : petroleum ether $=1: 4$ ) afforded product $3 \mathrm{~m}$ as a white solid $(47 \mathrm{mg}, 62 \%$ yield, $>20: 1 \mathrm{dr}): \mathrm{Rf}=0.4$ (EtOAc $:$ petroleum ether $=1$ : 1); ${ }^{1} \mathbf{H}$ NMR $\left(\mathrm{CDCl}_{3}, 400 \mathrm{MHz}\right) \delta: 8.05(\mathrm{~d}, J=7.5 \mathrm{~Hz}, 2 \mathrm{H}), 7.46(\mathrm{~d}, J=7.6 \mathrm{~Hz}, 2 \mathrm{H}), 7.13-7.09$ (m, 4H), 3.28-3.26 (m, 1H), 3.06-2.91 (m, 3H), 2.59-2.49 (m, 1H), 2.16-2.13 (m, 1H), $0.97(\mathrm{~s}, 6 \mathrm{H})$, $0.91(\mathrm{~s}, 6 \mathrm{H}) ;{ }^{13} \mathrm{C}$ NMR $\left(\mathrm{CDCl}_{3}, 100 \mathrm{MHz}\right) \delta: 171.8,152.3,137.2,135.8,130.1,129.4,128.7$, 127.9, 127.0, 125.5, 125, 1, 83.1, 41.4, 29.5, 25.2, 24.5, $24.2 \mathrm{ppm}$; HRMS (ESI-TOF) $\mathrm{m} / \mathrm{z}$ calcd for $\mathrm{C}_{23} \mathrm{H}_{28} \mathrm{BO}_{4}(\mathrm{M}+\mathrm{H})^{+}: 379.2085$, found 379.2088 .

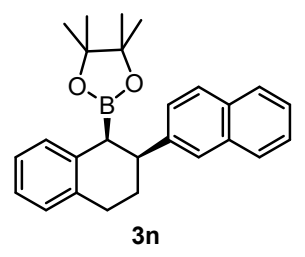

4,4,5,5-tetramethyl-2-(1,2,3,4-tetrahydro-[2,2'-binaphthalen]-1-yl)-1,3,2-dioxaborolane (3n): The general procedure A was followed using 1,2-dihydronaphthalene (1a, $26 \mathrm{mg}, 0.20 \mathrm{mmol})$ and 2,2'-binaphthalen]-1-yl diazonium tetrafluoroborate (2n, $73 \mathrm{mg}, 0.30 \mathrm{mmol})$. Purification of this material by chromatography on silica gel (EtOAc : petroleum ether $=1: 20$ ) afforded product 3n as a colorless solid (32 mg, 42\% yield, $>20: 1 \mathrm{dr}): \mathrm{Rf}=0.5$ (EtOAc $:$ petroleum ether $=1: 10) ;{ }^{1} \mathbf{H}$ NMR $\left(\mathrm{CDCl}_{3}, 400 \mathrm{MHz}\right) \delta: 7.80-7.76(\mathrm{~m}, 4 \mathrm{H}), 7.49(\mathrm{~d}, J=8.4 \mathrm{~Hz}, 1 \mathrm{H}), 7.45-7.39(\mathrm{~m}, 2 \mathrm{H})$, 7.14-7.09 (m, 4H), 3.36-3.33 (m, 1H), 3.15-3.14 (m, 1H), 3.06-2.95 (m, 2H), 2.71-2.60 (m, 1H), 2.24-2.21 (m, 1H), $0.90(\mathrm{~s}, 6 \mathrm{H}), 0.80(\mathrm{~s}, 6 \mathrm{H}) ;{ }^{13} \mathbf{C} \mathbf{~ N M R}\left(\mathrm{CDCl}_{3}, 100 \mathrm{MHz}\right) \delta: 143.3,137.8,136.1$, 133.5, 132.2, 129.4, 128.6, 127.7, 127.54, 127.52, 127.0, 125.7, 125.4, 125.3, 125.0, 124.95, 82.9, 41.3, 29.8, 25.5, 24.6, 24.1 ppm; HRMS (ESI-TOF) $m / z$ calcd for $\mathrm{C}_{26} \mathrm{H}_{30} \mathrm{BO}_{2}(\mathrm{M}+\mathrm{H})^{+}: 385.2343$, found 385.2339 .

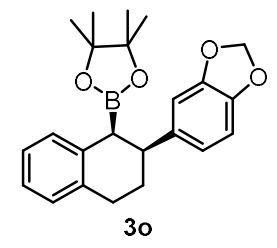

2-(2-(benzo $[d][1,3]$ dioxol-5-yl)-1,2,3,4-tetrahydronaphthalen-1-yl)-4,4,5,5-tetramethyl-1,3,2dioxaborolane (3o): The general procedure A was followed using 1,2-dihydronaphthalene (1a, 26 
$\mathrm{mg}, 0.20 \mathrm{mmol}$ ) and The correspondinge diazonium tetrafluoroborate $(\mathbf{2 o}, 71 \mathrm{mg}, 0.30 \mathrm{mmol})$. Purification of this material by chromatography on silica gel (EtOAc : petroleum ether $=1: 20$ ) afforded product $3 \mathrm{o}$ as a yellow solid ( $24 \mathrm{mg}, 32 \%$ yield, $>20: 1 \mathrm{dr}$ ): $\mathrm{Rf}=0.4$ (EtOAc : petroleum ether $=1: 10) ;{ }^{1} \mathbf{H}$ NMR $\left(\mathrm{CDCl}_{3}, 400 \mathrm{MHz}\right) \delta:$ 7.12-7.06 (m, 4H), $6.87(\mathrm{~s}, 1 \mathrm{H}), 6.80-6.73(\mathrm{~m}, 2 \mathrm{H})$, $5.91(\mathrm{~s}, 2 \mathrm{H}), 3.14-3.10(\mathrm{~m}, 1 \mathrm{H}), 2.98-2.88(\mathrm{~m}, 3 \mathrm{H}), 2.50-2.40(\mathrm{~m}, 1 \mathrm{H}), 2.06-2.03(\mathrm{~m}, 1 \mathrm{H}), 1.02(\mathrm{~s}$, 6H), $0.95(\mathrm{~s}, 6 \mathrm{H}) ;{ }^{13} \mathbf{C}$ NMR $\left(\mathrm{CDCl}_{3}, 100 \mathrm{MHz}\right) \delta: 147.2,145.5,139.9,137.6,136.0,129.4,128.6$, 125.4, 125.0, 120.4, 108.3, 107.9, 100.6, 82.9, 40.9, 29.8, 25.7, 24.6, 24.2 ppm; HRMS (ESI-TOF) $m / z$ calcd for $\mathrm{C}_{23} \mathrm{H}_{28} \mathrm{BO}_{4}(\mathrm{M}+\mathrm{H})^{+}: 378.2117$, found 378.2107.

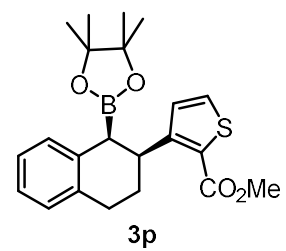

Methyl 3-(1-(4,4,5,5-tetramethyl-1,3,2-dioxaborolan-2-yl)-1,2,3,4-tetrahydronaphthalen-2yl)thiophene-2-carboxylate (3p): The general procedure A was followed using 1,2-dihydronaphthalene (1a, $26 \mathrm{mg}, 0.20 \mathrm{mmol}$ ) and The correspondinge diazonium tetrafluoroborate $(\mathbf{2 p}, 77 \mathrm{mg}, 0.30 \mathrm{mmol})$. Purification of this material by chromatography on silica gel (EtOAc : petroleum ether $=1: 20$ ) afforded product $\mathbf{3 p}$ as a yellow solid $(46 \mathrm{mg}, 58 \%$ yield, $>20: 1 \mathrm{dr}): \mathrm{Rf}=0.3($ EtOAc $:$ petroleum ether $=1: 10) ;{ }^{1} \mathbf{H} \mathbf{~ N M R}\left(\mathrm{CDCl}_{3}, 400 \mathrm{MHz}\right) \delta: 7.37$ $(\mathrm{d}, J=5.1 \mathrm{~Hz}, 1 \mathrm{H}), 7.20(\mathrm{~d}, J=5.1 \mathrm{~Hz}, 1 \mathrm{H}), 7.09-7.04(\mathrm{~m}, 4 \mathrm{H}), 4.10-4.06(\mathrm{~m}, 1 \mathrm{H}), 3.83(\mathrm{~s}, 3 \mathrm{H})$, $3.17(\mathrm{~d}, J=5.8 \mathrm{~Hz}, 1 \mathrm{H}), 2.95-2.91(\mathrm{~m}, 2 \mathrm{H}), 2.60-2.50(\mathrm{~m}, 1 \mathrm{H}), 2.03-2.00(\mathrm{~m}, 1 \mathrm{H}), 1.04(\mathrm{~s}, 6 \mathrm{H})$, 0.94 (s, 6H); ${ }^{13} \mathbf{C}$ NMR $\left(\mathrm{CDCl}_{3}, 100 \mathrm{MHz}\right) \delta: 162.7,154.4,137.5,135.8,129.62,129.56,129.2$, 128.8, 126.7, 125.3, 124.8, 83.0, 51.8, 35.9, 29.6, 26.6, 24.5, 24.4 ppm; HRMS (ESI-TOF) $\mathrm{m} / \mathrm{z}$ calcd for $\mathrm{C}_{22} \mathrm{H}_{28} \mathrm{BO}_{4} \mathrm{~S}(\mathrm{M}+\mathrm{H})^{+}: 399.1805$, found 399.1818 .

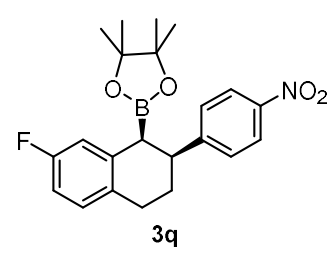

2-(7-fluoro-2-(4-nitrophenyl)-1,2,3,4-tetrahydronaphthalen-1-yl)-4,4,5,5-tetramethyl-1,3,2-di oxaborolane (3q): The general procedure A was followed using 6-fluoro-1,2-dihydronaphthalene (1b, $30 \mathrm{mg}, 0.20 \mathrm{mmol})$ and 4-nitrophenyldiazonium tetrafluoroborate $(\mathbf{2 a}, 71 \mathrm{mg}, 0.30 \mathrm{mmol})$. Purification of this material by chromatography on silica gel (EtOAc : petroleum ether $=1: 20$ ) afforded product $\mathbf{3 q}$ as a colorless solid $(68 \mathrm{mg}, 85 \%$ yield, $>20: 1 \mathrm{dr}): \mathrm{Rf}=0.5$ (EtOAc : petroleum ether $=1: 10) ;{ }^{1} \mathbf{H}$ NMR $\left(\mathrm{CDCl}_{3}, 400 \mathrm{MHz}\right) \delta: 8.16(\mathrm{~d}, J=8.2 \mathrm{~Hz}, 2 \mathrm{H}), 7.49(\mathrm{~d}, J=$ $8.2 \mathrm{~Hz}, 2 \mathrm{H}), 7.10-7.06(\mathrm{~m}, 1 \mathrm{H}), 6.87-6.78(\mathrm{~m}, 2 \mathrm{H})$ 3.29-3.27 (m, 1H), 3.01-2.84 (m, 3H), 2.55-2.45 (m, 1H), 2.15-2.13 (m, 1H), $0.98(\mathrm{~s}, 6 \mathrm{H}), 0.93(\mathrm{~s}, 6 \mathrm{H}) ;{ }^{13} \mathbf{C} \mathbf{~ N M R}\left(\mathrm{CDCl}_{3}, 100 \mathrm{MHz}\right) \delta$ : 162.1 159.7, 153.2, 146.5, 138.8, 138.7, 131.1, 130.63, 130.55, 128.6, 123.3, 114.8, 114.6, 112.6, 112.4, 83.4, 41.0, 28.6, 25.5, 24.5, 24.2 ppm; HRMS (ESI-TOF) $\mathrm{m} / z$ calcd for $\mathrm{C}_{22} \mathrm{H}_{26} \mathrm{BNO}_{4} \mathrm{~F}$ (M $+\mathrm{H})^{+}: 397.1975$, found 397.1983 . 


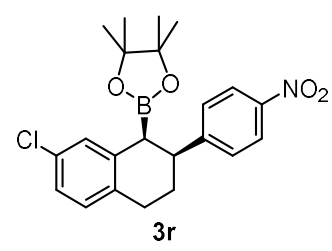

2-(7-chloro-2-(4-nitrophenyl)-1,2,3,4-tetrahydronaphthalen-1-yl)-4,4,5,5-tetramethyl-1,3,2-di oxaborolane (3r): The general procedure A was followed using 6-chloro-1,2-dihydronaphthalene (1c, $33 \mathrm{mg}, 0.20 \mathrm{mmol}$ ) and 4-nitrophenyldiazonium tetrafluoroborate (2a, $71 \mathrm{mg}, 0.30 \mathrm{mmol}$ ). Purification of this material by chromatography on silica gel (EtOAc : petroleum ether $=1: 20$ ) afforded product $3 \mathbf{r}$ as a colorless solid $(68 \mathrm{mg}, 82 \%$ yield, $>20: 1 \mathrm{dr}$ ): $\mathrm{Rf}=0.5$ (EtOAc : petroleum ether $=1: 10) ;{ }^{1} \mathbf{H}$ NMR $\left(\mathrm{CDCl}_{3}, 400 \mathrm{MHz}\right) \delta: 8.16(\mathrm{~d}, J=7.8 \mathrm{~Hz}, 2 \mathrm{H}), 7.49(\mathrm{~d}, J=$ $7.8 \mathrm{~Hz}, 2 \mathrm{H}), 7.14(\mathrm{~s}, 1 \mathrm{H}), 7.06(\mathrm{~s}, 2 \mathrm{H}), 3.28-3.25(\mathrm{~m}, 1 \mathrm{H}), 2.99-2.84(\mathrm{~m}, 3 \mathrm{H}), 2.56-2.46(\mathrm{~m}, 1 \mathrm{H})$, 2.15-2.12 (m, 1H), $0.98(\mathrm{~s}, 6 \mathrm{H}), 0.94(\mathrm{~s}, 6 \mathrm{H}) ;{ }^{13} \mathbf{C} \mathbf{~ N M R}\left(\mathrm{CDCl}_{3}, 100 \mathrm{MHz}\right) \delta: 153.1,146.5,138.7$, $134.0,131.1,130.6,128.5,128.3,125.5,123.3,83.5,41.0,28.7,25.2,24.6,24.1$ ppm; HRMS (ESI-TOF) $\mathrm{m} / z$ calcd for $\mathrm{C}_{22} \mathrm{H}_{26} \mathrm{BNO}_{4} \mathrm{Cl}(\mathrm{M}+\mathrm{H})^{+}: 413.1680$, found 413.1664 .

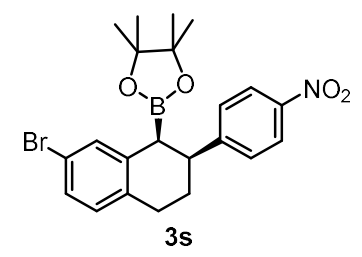

2-(7-bromo-2-(4-nitrophenyl)-1,2,3,4-tetrahydronaphthalen-1-yl)-4,4,5,5-tetramethyl-1,3,2-di oxaborolane (3s): The general procedure A was followed using 6-bromo-1,2-dihydronaphthalene (1d, $42 \mathrm{mg}, 0.20 \mathrm{mmol}$ ) and 4-nitrophenyldiazonium tetrafluoroborate (2a, $71 \mathrm{mg}, 0.30 \mathrm{mmol}$ ). Purification of this material by chromatography on silica gel (EtOAc : petroleum ether $=1: 20$ ) afforded product $3 \mathrm{~s}$ as a colorless solid $(46 \mathrm{mg}, 50 \%$ yield, $>20: 1 \mathrm{dr}$ ): $\mathrm{Rf}=0.5$ (EtOAc : petroleum ether $=1: 10) ;{ }^{1} \mathbf{H}$ NMR $\left(\mathrm{CDCl}_{3}, 400 \mathrm{MHz}\right) \delta: 8.16(\mathrm{~d}, J=7.8 \mathrm{~Hz}, 2 \mathrm{H}), 7.49(\mathrm{~d}, J=$ $7.8 \mathrm{~Hz}, 2 \mathrm{H}), 7.30(\mathrm{~s}, 1 \mathrm{H}), 7.21(\mathrm{~d}, J=8.0 \mathrm{~Hz}, 1 \mathrm{H}), 7.00(\mathrm{~d}, J=8.0 \mathrm{~Hz}, 1 \mathrm{H}), 3.27-3.25(\mathrm{~m}, 1 \mathrm{H})$, 2.99-2.82 (m, 3H), 2.56-2.45 (m, 1H), 2.15-2.12 (m, 1H), $0.98(\mathrm{~s}, 6 \mathrm{H}), 0.94(\mathrm{~s}, 6 \mathrm{H}) ;{ }^{13} \mathbf{C}$ NMR $\left(\mathrm{CDCl}_{3}, 100 \mathrm{MHz}\right) \delta: 153.1,146.5,139.2,134.6,131.3,131.0,128.5,128.4,123.3,119.1,83.5$, 41.0, 28.8, 25.2, 24.6, $24.1 \mathrm{ppm}$; HRMS (ESI-TOF) $\mathrm{m} / \mathrm{z}$ calcd for $\mathrm{C}_{22} \mathrm{H}_{24} \mathrm{BNO}_{4} \mathrm{Br}(\mathrm{M}-\mathrm{H})^{-}$: 455.1018, found 455.1018 .

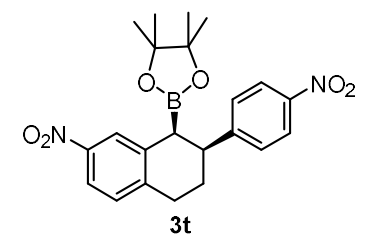

4,4,5,5-tetramethyl-2-(7-nitro-2-(4-nitrophenyl)-1,2,3,4-tetrahydronaphthalen-1-yl)-1,3,2-dio xaborolane (3t): The general procedure A was followed using 6-nitro-1,2-dihydronaphthalene (1e, $35 \mathrm{mg}, 0.20 \mathrm{mmol}$ ) and 4-nitrophenyldiazonium tetrafluoroborate (2a, $71 \mathrm{mg}, 0.30 \mathrm{mmol}$ ). Purification of this material by chromatography on silica gel (EtOAc : petroleum ether $=1: 20$ ) afforded product $3 \mathrm{t}$ as a yellow solid $(80 \mathrm{mg}, 94 \%$ yield, $>20: 1 \mathrm{dr}$ ): $\mathrm{Rf}=0.4$ (EtOAc : petroleum ether = 1: 10); ${ }^{1} \mathbf{H}$ NMR $\left(\mathrm{CDCl}_{3}, 400 \mathrm{MHz}\right) \delta: 8.18(\mathrm{~d}, J=7.9 \mathrm{~Hz}, 2 \mathrm{H}), 8.06(\mathrm{~s}, 1 \mathrm{H}), 7.95(\mathrm{~d}, J=$ $8.4 \mathrm{~Hz}, 1 \mathrm{H}), 7.50(\mathrm{~d}, J=7.9 \mathrm{~Hz}, 2 \mathrm{H}), 7.28(\mathrm{~d}, J=10.1 \mathrm{~Hz}, 1 \mathrm{H}), 3.35-3.33(\mathrm{~m}, 1 \mathrm{H}), 3.13-2.96(\mathrm{~m}$, 3H), 2.59-2.49 (m, 1H), 2.22-2.19 (m, 1H), $0.97(\mathrm{~s}, 6 \mathrm{H}), 0.94(\mathrm{~s}, 6 \mathrm{H}) ;{ }^{13} \mathbf{C} \mathbf{~ N M R}\left(\mathrm{CDCl}_{3}, 100\right.$ 
MHz) $\delta: 152.4,146.6,146.1,143.5,138.7,130.2,128.5,123.7,123.4,120.3,83.7,40.7,29.4$, 25.0, 24.5, 24.1 ppm; HRMS (ESI-TOF) $m / z$ calcd for $\mathrm{C}_{22} \mathrm{H}_{24} \mathrm{BN}_{2} \mathrm{O}_{6}(\mathrm{M}-\mathrm{H})^{-}: 423.1732$, found 423.1752 .

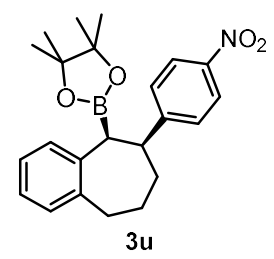

4,4,5,5-tetramethyl-2-(6-(4-nitrophenyl)-6,7,8,9-tetrahydro-5H-benzo[7]annulen-5-yl)-1,3,2-d ioxaborolane (3u): The general procedure $A$ was followed using 6,7-dihydro-5H-benzo[7]annulene (1f, $29 \mathrm{mg}, \quad 0.20 \mathrm{mmol}$ ) and 4-nitrophenyldiazonium tetrafluoroborate $(\mathbf{2 a}, 71 \mathrm{mg}, 0.30 \mathrm{mmol})$. Purification of this material by chromatography on silica gel (EtOAc : petroleum ether $=1: 20$ ) afforded product $3 \mathbf{u}$ as a colorless solid $(57 \mathrm{mg}, 72 \%$ yield, $>20: 1 \mathrm{dr}): \mathrm{Rf}=0.5($ EtOAc $:$ petroleum ether $=1: 10) ;{ }^{1} \mathbf{H} \mathbf{~ N M R}\left(\mathrm{CDCl}_{3}, 400 \mathrm{MHz}\right) \delta: 8.14$ $(\mathrm{d}, J=7.9 \mathrm{~Hz}, 2 \mathrm{H}), 7.53(\mathrm{~d}, J=8.4 \mathrm{~Hz}, 2 \mathrm{H}), 7.14-7.11(\mathrm{~m}, 4 \mathrm{H}), 3.16-3.10(\mathrm{~m}, 1 \mathrm{H}), 3.00-2.97(\mathrm{~m}$, 1H), 2.79-2.62 (m, 3H), $2.10(\mathrm{~m}, 1 \mathrm{H}), 1.99-1.96(\mathrm{~m}, 1 \mathrm{H}), 1.51-1.45(\mathrm{~m}, 1 \mathrm{H}), 1.18(\mathrm{~s}, 6 \mathrm{H}), 1.13(\mathrm{~s}$, $6 \mathrm{H}) ;{ }^{13} \mathbf{C}$ NMR $\left(\mathrm{CDCl}_{3}, 100 \mathrm{MHz}\right) \delta: 156.3,146.2,142.8,142.4,130.2,129.8,128.5,126.3,126.0$, 123.4, 83.4, 47.5, 35.7, 34.0, 28.0, 24.8, 24.5 ppm; HRMS (ESI-TOF) $m / z$ calcd for $\mathrm{C}_{23} \mathrm{H}_{27} \mathrm{BNO}_{4}$ $(\mathrm{M}-\mathrm{H})^{-}: 391.2069$, found 391.2085.

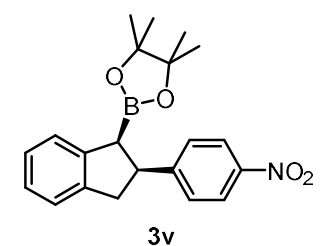

4,4,5,5-tetramethyl-2-(2-(4-nitrophenyl)-2,3-dihydro-1H-inden-1-yl)-1,3,2-dioxaborolane (3v): The general procedure A was followed using $1 H$-indene $(1 \mathrm{~g}, 23 \mathrm{mg}, 0.20 \mathrm{mmol})$ and 4-nitrophenyldiazonium tetrafluoroborate (2a, $71 \mathrm{mg}, 0.30 \mathrm{mmol})$. Purification of this material by chromatography on silica gel (EtOAc : petroleum ether $=1: 20$ ) afforded product $\mathbf{3 v}$ as a colorless solid (49 mg, 67\% yield, >20:1 dr): $\mathrm{Rf}=0.5$ (EtOAc : petroleum ether $=1: 10) ;{ }^{1} \mathbf{H} \mathbf{~ N M R}\left(\mathrm{CDCl}_{3}\right.$, $400 \mathrm{MHz}) \delta: 8.08(\mathrm{~d}, J=8.2 \mathrm{~Hz}, 2 \mathrm{H}), 7.36(\mathrm{~d}, J=8.2 \mathrm{~Hz}, 2 \mathrm{H}), 7.34-7.32(\mathrm{~m}, 1 \mathrm{H}), 7.28-7.26(\mathrm{~m}$, $1 \mathrm{H}), 7.22-7.15(\mathrm{~m}, 2 \mathrm{H}), 4.00-3.95(\mathrm{~m}, 1 \mathrm{H}), 3.43-3.38(\mathrm{~m}, 1 \mathrm{H}), 3.32-3.30(\mathrm{~d}, J=8.4 \mathrm{~Hz}, 1 \mathrm{H})$, 3.25-3.20 (m, 1H), 0.92 (s, 12H); ${ }^{13} \mathbf{C}$ NMR $\left(\mathrm{CDCl}_{3}, 100 \mathrm{MHz}\right) \delta: 153.3,146.4,143.9,142.4$, 128.2, 126.6, 126.0, 124.5, 124.4, 123.3, 83.3, 47.0, 39.4, 24.4 ppm; HRMS (ESI-TOF) $\mathrm{m} / z$ calcd for $\mathrm{C}_{21} \mathrm{H}_{23} \mathrm{BNO}_{4}(\mathrm{M}-\mathrm{H}): 363.1757$, found 363.1757 .

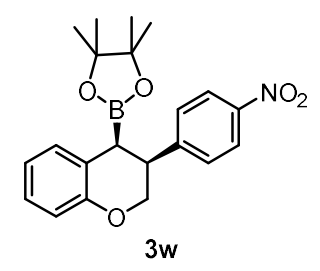

4,4,5,5-tetramethyl-2-(3-(4-nitrophenyl)chroman-4-yl)-1,3,2-dioxaborolane (3w): The general procedure A was followed using $2 H$-chromene $(\mathbf{1 h}, 26 \mathrm{mg}, 0.20 \mathrm{mmol})$ and 4-nitrophenyldiazonium tetrafluoroborate (2a, $71 \mathrm{mg}, 0.30 \mathrm{mmol})$. Purification of this material by chromatography on silica gel (EtOAc : petroleum ether $=1: 20$ ) afforded product $\mathbf{3 w}$ as a colorless solid (36 mg, 47\% yield, $>20: 1 \mathrm{dr}): \mathrm{Rf}=0.5($ EtOAc $:$ petroleum ether $=1: 10) ;{ }^{1} \mathbf{H}$ 
NMR $\left(\mathrm{CDCl}_{3}, 400 \mathrm{MHz}\right) \delta: 7.55(\mathrm{~d}, J=8.0 \mathrm{~Hz}, 2 \mathrm{H}), 7.38(\mathrm{~d}, J=8.0 \mathrm{~Hz}, 2 \mathrm{H}), 7.15-7.08(\mathrm{~m}, 2 \mathrm{H})$, 6.89-6.85 (m, 2H), $4.65(\mathrm{t}, J=9.8 \mathrm{~Hz}, 1 \mathrm{H}), 4.46(\mathrm{~d}, J=10.2 \mathrm{~Hz}, 1 \mathrm{H}), 3.47$ (m, 1H), 3.04 (d, $J=$ $8.4 \mathrm{~Hz}, 1 \mathrm{H}), 0.98(\mathrm{~s}, 6 \mathrm{H}), 0.96(\mathrm{~s}, 6 \mathrm{H}) ;{ }^{13} \mathbf{C} \mathbf{~ N M R}\left(\mathrm{CDCl}_{3}, 100 \mathrm{MHz}\right) \delta: 154.0,145.9,129.5,128.0$, 126.9, 125.3,125.2, 122.5, 120.5, 116.9, 83.6, 67.7, 39.0, 24.5, $24.2 \mathrm{ppm}$; HRMS (ESI-TOF) $\mathrm{m} / \mathrm{z}$ calcd for $\mathrm{C}_{21} \mathrm{H}_{25} \mathrm{BNO}_{5}(\mathrm{M}+\mathrm{H})^{+}:$381.1862, found 381.1853 .

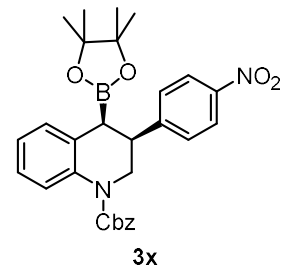

Benzyl3-(4-nitrophenyl)-4-(4,4,5,5-tetramethyl-1,3,2-dioxaborolan-2-yl)-3,4-dihydroquinolin e-1(2H)-carboxylate (3x): The general procedure A was followed using benzyl quinoline-1 $(2 H)$-carboxylate $(\mathbf{1 i}, \quad 53 \mathrm{mg}, \quad 0.20 \mathrm{mmol})$ and 4-nitrophenyldiazonium tetrafluoroborate $(\mathbf{2 a}, 71 \mathrm{mg}, 0.30 \mathrm{mmol})$. Purification of this material by chromatography on silica gel (EtOAc : petroleum ether $=1: 15)$ afforded product $\mathbf{3 x}$ as a yellow solid $(47 \mathrm{mg}, 46 \%$ yield, $>20: 1 \mathrm{dr}): \mathrm{Rf}=0.3($ EtOAc : petroleum ether $=1: 10) ;{ }^{1} \mathbf{H} \mathbf{~ N M R}\left(\mathrm{CDCl}_{3}, 400 \mathrm{MHz}\right) \delta: 8.05$ $(\mathrm{d}, J=8.0 \mathrm{~Hz}, 2 \mathrm{H}), 7.70(\mathrm{~m}, 1 \mathrm{H}), 7.39$ (d, $J=8.0 \mathrm{~Hz}, 2 \mathrm{H}), 7.29-7.16(\mathrm{~m}, 7 \mathrm{H}), 7.09-7.05(\mathrm{~m}, 1 \mathrm{H})$, 5.11-5.03 (m, 2H), 4.35-4.30 (m, 1H), $4.00(\mathrm{~d}, J=12.3 \mathrm{~Hz}, 1 \mathrm{H}), 3.51(\mathrm{~m}, 1 \mathrm{H}), 3.09-3.08(\mathrm{~m}, 1 \mathrm{H})$, $0.98(\mathrm{~s}, 6 \mathrm{H}), 0.90(\mathrm{~s}, 6 \mathrm{H}) ;{ }^{13} \mathbf{C}$ NMR $\left(\mathrm{CDCl}_{3}, 100 \mathrm{MHz}\right) \delta: 154.6,150.1,146.8,137.4,136.1$, 129.6, 129.0, 128.7, 128.4, 128.1, 125.8, 124.6, 124.1, 123.4, 83.7, 67.5, 48.0, 41.3, 24.4, 24.3 ppm; HRMS (ESI-TOF) $m / z$ calcd for $\mathrm{C}_{29} \mathrm{H}_{32} \mathrm{BN}_{2} \mathrm{O}_{6}(\mathrm{M}+\mathrm{H})^{+}: 515.2358$, found 515.2368.

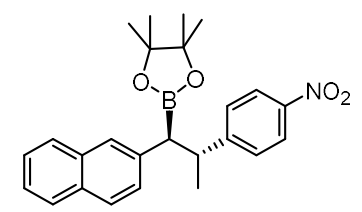

$3 y$

4,4,5,5-tetramethyl-2-((1S,2S)-1-(naphthalen-2-yl)-2-(4-nitrophenyl)propyl)-1,3,2-dioxaborol ane (3y): The general procedure A was followed using (E)-2-(prop-1-en-1-yl)naphthalene (1jj, 34 $\mathrm{mg}, 0.20 \mathrm{mmol}$ ) and 4-nitrophenyldiazonium tetrafluoroborate (2a, $71 \mathrm{mg}, 0.30 \mathrm{mmol}$ ). Purification of this material by chromatography on silica gel (EtOAc : petroleum ether $=1: 20$ ) afforded product $3 \mathbf{y}$ as a colorless solid $(56 \mathrm{mg}, 67 \%$ yield, $>20: 1 \mathrm{dr}$ ): $\mathrm{Rf}=0.6$ (EtOAc : petroleum ether $=1: 10) ;{ }^{1} \mathbf{H}$ NMR $\left(\mathrm{CDCl}_{3}, 400 \mathrm{MHz}\right) \delta: 7.82-7.76(\mathrm{~m}, 4 \mathrm{H}), 7.58-7.56(\mathrm{~m}, 2 \mathrm{H})$, 7.52-7.41 (m, 5H), 3.42-3.34 (m, 1H), $2.71(\mathrm{~d}, J=11.7 \mathrm{~Hz}, 1 \mathrm{H}), 1.08(\mathrm{~d}, J=6.8 \mathrm{~Hz}, 3 \mathrm{H}), 0.88(\mathrm{~s}$, $12 \mathrm{H}) ;{ }^{13} \mathbf{C ~ N M R}\left(\mathrm{CDCl}_{3}, 100 \mathrm{MHz}\right) \delta: 151.7,138.5,133.8,132.0,128.0,127.9,127.59,127.56$, 127.5, 127.4, 125.8, 125.14, 125.10, 83.3, 42.8, 24.3, 24.0, 21.8 ppm; HRMS (ESI-TOF) $\mathrm{m} / \mathrm{z}$ calcd for $\mathrm{C}_{25} \mathrm{H}_{27} \mathrm{BNO}_{4}(\mathrm{M}-\mathrm{H}): 415.2069$, found 415.2075 .

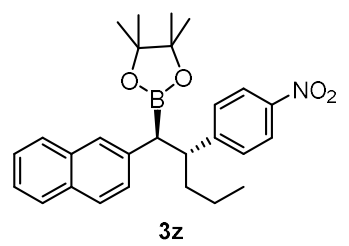

4,4,5,5-tetramethyl-2-((1S,2R)-1-(naphthalen-2-yl)-2-(4-nitrophenyl)pentyl)-1,3,2-dioxaborol ane (3z): The general procedure A was followed using $(E)$-2-(pent-1-en-1-yl)naphthalene (1k, 39 $\mathrm{mg}, 0.20 \mathrm{mmol}$ ) and 4-nitrophenyldiazonium tetrafluoroborate (2a, $71 \mathrm{mg}, 0.30 \mathrm{mmol})$. 
Purification of this material by chromatography on silica gel (EtOAc : petroleum ether $=1: 20$ ) afforded product $\mathbf{3 z}$ as a colorless solid $(35 \mathrm{mg}, 39 \%$ yield, $>20: 1 \mathrm{dr}): \mathrm{Rf}=0.6($ EtOAc : petroleum ether $=1: 10) ;{ }^{1} \mathbf{H}$ NMR $\left(\mathrm{CDCl}_{3}, 400 \mathrm{MHz}\right) \delta: 8.19(\mathrm{~d}, J=8.0 \mathrm{~Hz}, 2 \mathrm{H}), 7.83-7.81(\mathrm{~m}$, $3 \mathrm{H}), 7.76(\mathrm{~s}, 1 \mathrm{H}), 7.52-7.42(\mathrm{~m}, 5 \mathrm{H}), 3.31(\mathrm{t}, J=11.2 \mathrm{~Hz}, 1 \mathrm{H}), 2.78(\mathrm{~d}, J=12.0 \mathrm{~Hz}, 1 \mathrm{H})$, 1.54-1.50 (m, 1H), 1.40-1.31 (m, 1H), 1.03-0.91 (m, 2H), $0.87(\mathrm{~s}, 6 \mathrm{H}), 0.86(\mathrm{~s}, 6 \mathrm{H}), 0.64(\mathrm{t}, J=$ $7.2 \mathrm{~Hz}, 3 \mathrm{H}) ;{ }^{13} \mathbf{C} \mathbf{~ N M R}\left(\mathrm{CDCl}_{3}, 100 \mathrm{MHz}\right) \delta: 153.9,146.5,138.0,133.8,132.1,129.3,128.0$, 127.6, 127.52, 127.47, 125.9, 125.2, 123.3, 83.4, 48.0, 37.2, 24.3, 24.1, 20.2, 13.7 ppm; HRMS (ESI-TOF) $m / z$ calcd for $\mathrm{C}_{27} \mathrm{H}_{33} \mathrm{BNO}_{4}(\mathrm{M}+\mathrm{H})^{+}: 445.2539$, found 445.2550 .

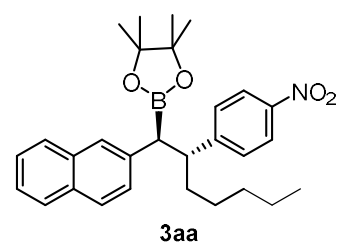

4,4,5,5-tetramethyl-2-((1S,2R)-1-(naphthalen-2-yl)-2-(4-nitrophenyl)heptyl)-1,3,2-dioxaborol ane (3aa): The general procedure A was followed using (E)-2-(hept-1-en-1-yl)naphthalene (11, 45 $\mathrm{mg}, 0.20 \mathrm{mmol}$ ) and 4-nitrophenyldiazonium tetrafluoroborate (2a, $71 \mathrm{mg}, 0.30 \mathrm{mmol})$. Purification of this material by chromatography on silica gel (EtOAc : petroleum ether $=1: 20$ ) afforded product 3aa as a colorless solid $(37 \mathrm{mg}, 39 \%$ yield, $>20: 1 \mathrm{dr}$ ): $\mathrm{Rf}=0.6$ (EtOAc : petroleum ether $=1: 10) ;{ }^{1} \mathbf{H}$ NMR $\left(\mathrm{CDCl}_{3}, 400 \mathrm{MHz}\right) \delta: 8.19(\mathrm{~d}, J=8.0 \mathrm{~Hz}, 2 \mathrm{H}), 7.81-7.80(\mathrm{~m}$, $3 \mathrm{H}), 7.75(\mathrm{~s}, 1 \mathrm{H}), 7.52-7.42(\mathrm{~m}, 5 \mathrm{H}), 3.29(\mathrm{t}, J=11.2 \mathrm{~Hz}, 1 \mathrm{H}), 2.78(\mathrm{~d}, J=11.6 \mathrm{~Hz}, 1 \mathrm{H}), 1.56(\mathrm{~m}$, $1 \mathrm{H}), 1.41-1.33(\mathrm{~m}, 1 \mathrm{H}), 1.04-0.90(\mathrm{~m}, 6 \mathrm{H}), 0.87(\mathrm{~s}, 6 \mathrm{H}), 0.86(\mathrm{~s}, 6 \mathrm{H}), 0.69(\mathrm{t}, J=5.2 \mathrm{~Hz}, 3 \mathrm{H})$; ${ }^{13} \mathbf{C}$ NMR $\left(\mathrm{CDCl}_{3}, 100 \mathrm{MHz}\right) \delta: 153.9,146.5,138.0,133.8,132.1,129.3,128.0,127.6,127.52$, $127.47,125.9,125.2,123.3,83.4,48.2,34.9,31.5,26.7,24.4,24.1,22.4,13.9$ ppm; HRMS (ESI-TOF) $m / z$ calcd for $\mathrm{C}_{29} \mathrm{H}_{37} \mathrm{BNO}_{4}(\mathrm{M}+\mathrm{H})^{+}: 473.2852$, found 473.2862 .

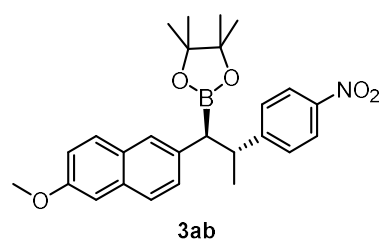

2-((1S,2R)-1-(6-methoxynaphthalen-2-yl)-2-(4-nitrophenyl)propyl)-4,4,5,5-tetramethyl-1,3,2dioxaborolane (3ab): The general procedure A was followed using (E)-2-methoxy-6-(prop-1-en-1-yl)naphthalene $\quad(\mathbf{1 m}, \quad 40 \quad \mathrm{mg}, \quad 0.20 \quad \mathrm{mmol}) \quad$ and 4-nitrophenyldiazonium tetrafluoroborate (2a, $71 \mathrm{mg}, 0.30 \mathrm{mmol})$. Purification of this material by chromatography on silica gel (EtOAc : petroleum ether $=1: 20$ ) afforded product 3ab as a colorless solid (43 mg, 48\% yield, $>20: 1 \mathrm{dr}): \mathrm{Rf}=0.5($ EtOAc $:$ petroleum ether $=1: 10) ;{ }^{1} \mathbf{H}$ NMR $\left(\mathrm{CDCl}_{3}, 400 \mathrm{MHz}\right) \delta: 8.18(\mathrm{~d}, J=7.7 \mathrm{~Hz}, 2 \mathrm{H}), 7.71-7.67(\mathrm{~m}, 3 \mathrm{H}), 7.52(\mathrm{~d}, J=7.7 \mathrm{~Hz}, 2 \mathrm{H})$, $7.43(\mathrm{~d}, J=8.3 \mathrm{~Hz}, 1 \mathrm{H}), 7.15-7.13(\mathrm{~m}, 2 \mathrm{H}), 3.92(\mathrm{~s}, 3 \mathrm{H}), 3.45-3.39(\mathrm{~m}, 1 \mathrm{H}), 2.66(\mathrm{~d}, J=11.5 \mathrm{~Hz}$, $1 \mathrm{H}), 1.08(\mathrm{~d}, J=6.4 \mathrm{~Hz}, 3 \mathrm{H}), 0.92(\mathrm{~s}, 6 \mathrm{H}), 0.89(\mathrm{~s}, 6 \mathrm{H}) ;{ }^{13} \mathbf{C} \mathbf{~ N M R}\left(\mathrm{CDCl}_{3}, 100 \mathrm{MHz}\right) \delta: 157.3$, 155.7, 146.4, 135.5, 133.1, 129.3, 129.0, 128.5, 128.0, 127.4, 126.9, 123.5, 118.7, 105.6, 83.4, 55.3, 42.8, 24.4, 24.2, $21.6 \mathrm{ppm}$; HRMS (ESI-TOF) $m / z$ calcd for $\mathrm{C}_{26} \mathrm{H}_{31} \mathrm{BNO}_{5}(\mathrm{M}+\mathrm{H})^{+}$: 447.2332, found 447.2306. 


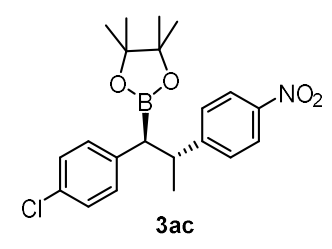

2-((1S,2R)-1-(4-chlorophenyl)-2-(4-nitrophenyl)propyl)-4,4,5,5-tetramethyl-1,3,2-dioxaborola ne (3ac): The general procedure A was followed using (E)-1-chloro-4-(prop-1-en-1-yl)benzene (1n, $30 \mathrm{mg}, 0.20 \mathrm{mmol})$ and 4-nitrophenyldiazonium tetrafluoroborate (2a, $71 \mathrm{mg}, 0.30 \mathrm{mmol})$. Purification of this material by chromatography on silica gel (EtOAc : petroleum ether $=1: 20$ ) afforded product $3 \mathrm{ac}$ as a colorless solid $(42 \mathrm{mg}, 53 \%$ yield, $>20: 1 \mathrm{dr}$ ): $\mathrm{Rf}=0.6$ (EtOAc : petroleum ether $=1: 10) ;{ }^{1} \mathbf{H}$ NMR $\left(\mathrm{CDCl}_{3}, 400 \mathrm{MHz}\right) \delta: 8.17(\mathrm{~d}, J=8.1 \mathrm{~Hz}, 2 \mathrm{H}), 7.46(\mathrm{~d}, J=$ $8.1 \mathrm{~Hz}, 2 \mathrm{H}), 7.29-7.22(\mathrm{~m}, 4 \mathrm{H}), 3.32-3.24(\mathrm{~m}, 1 \mathrm{H}), 2.50(\mathrm{~d}, J=11.4 \mathrm{~Hz}, 1 \mathrm{H}), 1.06(\mathrm{~d}, J=6.8 \mathrm{~Hz}$, $3 \mathrm{H}), 0.92(\mathrm{~s}, 6 \mathrm{H}), 0.91(\mathrm{~s}, 6 \mathrm{H}) ;{ }^{13} \mathbf{C}$ NMR $\left(\mathrm{CDCl}_{3}, 100 \mathrm{MHz}\right) \delta: 155.1,146.4,138.9,131.7,130.4$, 128.6, 128.4, 123.5, 83.5, 42.8, 24.3, 24.1, $21.4 \mathrm{ppm}$; HRMS (ESI-TOF) $\mathrm{m} / \mathrm{z}$ calcd for $\mathrm{C}_{21} \mathrm{H}_{26} \mathrm{BNO}_{4} \mathrm{Cl}(\mathrm{M}+\mathrm{H})^{+}: 401.1680$, found 401.1681 .

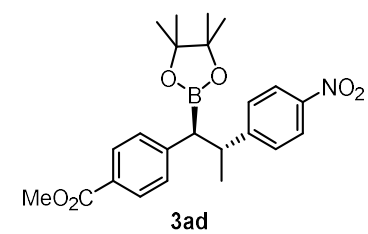

methyl 4-((1S,2R)-2-(4-nitrophenyl)-1-(4,4,5,5-tetramethyl-1,3,2-dioxaborolan-2-yl)propyl) benzoate (3ad): The general procedure $A$ was followed using methyl (E)-4-(prop-1-en-1-yl)benzoate $\quad(\mathbf{1 0}, \quad 35 \mathrm{mg}, \quad 0.20 \mathrm{mmol})$ and 4-nitrophenyldiazonium tetrafluoroborate $(\mathbf{2 a}, 71 \mathrm{mg}, 0.30 \mathrm{mmol})$. Purification of this material by chromatography on silica gel (EtOAc : petroleum ether $=1: 10)$ afforded product 3ad as a colorless solid (42 $\mathrm{mg}, 49 \%$ yield, $>20: 1 \mathrm{dr}): \mathrm{Rf}=0.2($ EtOAc $:$ petroleum ether $=1: 10) ; \mathbf{3 m a :}{ }^{1} \mathbf{H} \mathbf{N M R}\left(\mathrm{CDCl}_{3}, 400 \mathrm{MHz}\right) \delta$ : $8.17(\mathrm{~d}, J=7.8 \mathrm{~Hz}, 2 \mathrm{H}), 7.99(\mathrm{~d}, J=7.5 \mathrm{~Hz}, 2 \mathrm{H}), 7.48$ (d, $J=7.8 \mathrm{~Hz}, 2 \mathrm{H}), 7.38(\mathrm{~d}, J=7.5 \mathrm{~Hz}$, 2H), 3,91 (s, 3H), 3.39-3.34 (m, 1H), 2.62 (d, $J=11.2 \mathrm{~Hz}, 1 \mathrm{H}), 1.06$ (d, $J=6.5 \mathrm{~Hz}, 3 \mathrm{H}), 0.91$ (s, $6 \mathrm{H}), 0.90(\mathrm{~s}, 6 \mathrm{H}) ;{ }^{13} \mathrm{C}$ NMR $\left(\mathrm{CDCl}_{3}, 100 \mathrm{MHz}\right) \delta: 167.1,154.9,146.5,146.2,129.8,129.0,128.4$, $128.0,123.5,83.6,52.0,42.6,24.3,24.1,21.5 \mathrm{ppm}$; HRMS (ESI-TOF) $\mathrm{m} / z$ calcd for $\mathrm{C}_{23} \mathrm{H}_{29} \mathrm{BNO}_{6}$ $(\mathrm{M}+\mathrm{H})^{+}: 425,2124$, found 425.2119 .

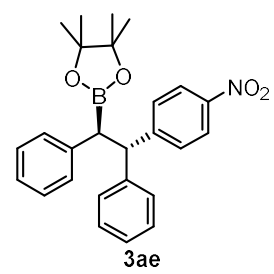

4,4,5,5-tetramethyl-2-((1S,2S)-2-(4-nitrophenyl)-1,2-diphenylethyl)-1,3,2-dioxaborolane (3ae): The general procedure A was followed using methyl $(E)$-1,2-diphenylethene (1p, $36 \mathrm{mg}, 0.20$ $\mathrm{mmol}$ ) and 4-nitrophenyldiazonium tetrafluoroborate (2a, $71 \mathrm{mg}, 0.30 \mathrm{mmol})$. Purification of this material by chromatography on silica gel (EtOAc : petroleum ether $=1: 20$ ) afforded product 3ae as a colorless solid $(50 \mathrm{mg}, 58 \%$ yield, $>20: 1 \mathrm{dr}): \mathrm{Rf}=0.5$ (EtOAc : petroleum ether $=1: 10) ;{ }^{1} \mathbf{H}$ NMR $\left(\mathrm{CDCl}_{3}, 400 \mathrm{MHz}\right) \delta: 8.16(\mathrm{~d}, J=8.0 \mathrm{~Hz}, 2 \mathrm{H}), 7.61(\mathrm{~d}, J=8.0 \mathrm{~Hz}, 2 \mathrm{H}), 7.18-7.02(\mathrm{~m}, 10 \mathrm{H})$, $4.59(\mathrm{~d}, J=12.6 \mathrm{~Hz}, 1 \mathrm{H}), 3.28(\mathrm{~d}, J=12.6 \mathrm{~Hz}, 1 \mathrm{H}), 0.99(\mathrm{~s}, 6 \mathrm{H}), 0.90(\mathrm{~s}, 6 \mathrm{H}) ;{ }^{13} \mathbf{C} \mathbf{~ N M R}\left(\mathrm{CDCl}_{3}\right.$, $100 \mathrm{MHz}) \delta:$ 153.0, 146.4, 142.3, 139.5, 129.02, 128.96, 128.4, 128.3, 127.9, 126.4, 125.7, 123.6, 
83.7, 53.8, 24.3, $24.2 \mathrm{ppm}$; HRMS (ESI-TOF) $\mathrm{m} / z$ calcd for $\mathrm{C}_{26} \mathrm{H}_{29} \mathrm{BNO}_{4}(\mathrm{M}+\mathrm{H})^{+}$: 429.2226, found 429.2218 .

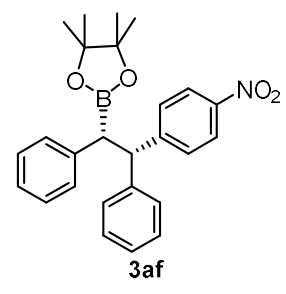

4,4,5,5-tetramethyl-2-((1R,2S)-2-(4-nitrophenyl)-1,2-diphenylethyl)-1,3,2-dioxaborolane (3af): The general procedure A was followed using methyl (Z)-1,2-diphenylethene (1q, $36 \mathrm{mg}, 0.20$ $\mathrm{mmol}$ ) and 4-nitrophenyldiazonium tetrafluoroborate (2a, $71 \mathrm{mg}, 0.30 \mathrm{mmol})$. Purification of this material by chromatography on silica gel (EtOAc : petroleum ether $=1: 20$ ) afforded product 3af as a colorless solid $(57 \mathrm{mg}, 66 \%$ yield, $>20: 1 \mathrm{dr}): \mathrm{Rf}=0.5($ EtOAc $:$ petroleum ether $=1: 10) ;{ }^{1} \mathbf{H}$ NMR $\left(\mathrm{CDCl}_{3}, 400 \mathrm{MHz}\right) \delta: 7.92(\mathrm{~d}, J=8.0 \mathrm{~Hz}, 2 \mathrm{H}), 7.43(\mathrm{~d}, J=7.6 \mathrm{~Hz}, 2 \mathrm{H}), 7.31(\mathrm{t}, J=7.2 \mathrm{~Hz}$, $2 \mathrm{H}), 7.22-7.12(\mathrm{~m}, 7 \mathrm{H}), 7.07-7.04(\mathrm{~m}, 1 \mathrm{H}), 4.59(\mathrm{~d}, J=12.8 \mathrm{~Hz}, 1 \mathrm{H}), 3.26(\mathrm{~d}, J=12.8 \mathrm{~Hz}, 1 \mathrm{H})$, 0.97 (s, 6H), 0.89 (s, 6H); ${ }^{13} \mathbf{C}$ NMR $\left(\mathrm{CDCl}_{3}, 100 \mathrm{MHz}\right) \delta: 152.0,146.0,143.3,139.5,128.9$, 128.7, 128.4, 128.1, 126.9, 125.8, 123.3, 83.6, 53.9, 24.2, 24.1 ppm; HRMS (ESI-TOF) $\mathrm{m} / \mathrm{zcalcd}$ for $\mathrm{C}_{26} \mathrm{H}_{27} \mathrm{BNO}_{4}(\mathrm{M}-\mathrm{H})^{-}: 427.2096$, found 427.2077.

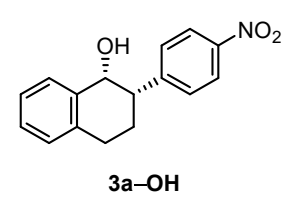

(1R, 2S)-2-(4-nitrophenyl)-1,2,3,4-tetrahydronaphthalen-1-ol (3a-OH): The general procedure B was followed using 1,2-dihydronaphthalene (1a, $13 \mathrm{mg}, 0.10 \mathrm{mmol})$, Ligand ((R)-L12,10 $\mathrm{mg}$, $0.01 \mathrm{mmol}$ ) and 4-nitrophenyldiazonium tetrafluoroborate (2a, $47 \mathrm{mg}, 0.20 \mathrm{mmol})$. Purification of this material by chromatography on silica gel (EtOAc : petroleum ether $=1: 10)$ afforded product 3a-OH as a White solid ( $5 \mathrm{mg}, 20 \%$ yield, $>20: 1 \mathrm{dr}$, 93:7 er): $\mathrm{Rf}=0.2$ (EtOAc : petroleum ether $=$ $1: 5) ;{ }^{1} \mathbf{H}$ NMR $\left(\mathrm{CDCl}_{3}, 400 \mathrm{MHz}\right) \delta: 8.22(\mathrm{~d}, J=7.9 \mathrm{~Hz}, 2 \mathrm{H}), 7.52(\mathrm{~d}, J=7.9 \mathrm{~Hz}, 2 \mathrm{H}), 7.34-7.21$ $(\mathrm{m}, 4 \mathrm{H}), 4.82(\mathrm{~s} 1 \mathrm{H}), 3.19(\mathrm{~d}, J=12.8 \mathrm{~Hz}, 2 \mathrm{H}), 3.10-3.04(\mathrm{~m}, 1 \mathrm{H}), 2.99-2.91(\mathrm{~m}, 1 \mathrm{H}), 2.54-2.43$ $(\mathrm{m}, 1 \mathrm{H}), 2.00-1.97(\mathrm{~m}, 1 \mathrm{H}), 1.55(\mathrm{~s} 1 \mathrm{H}) ;{ }^{13} \mathbf{C} \mathbf{~ N M R}\left(\mathrm{CDCl}_{3}, 100 \mathrm{MHz}\right) \delta: 150.8,146.8,137.5$, 136.2, 130.0, 129.3, 129.2, 128.5, 126.5, 123.5, 70.9, 46.1, 29.3, 21.7 ppm; HRMS (ESI-TOF) $m / z$ calcd for $\mathrm{C}_{16} \mathrm{H}_{16} \mathrm{O}_{3} \mathrm{~N}(\mathrm{M}+\mathrm{H})^{+}: 270.1130$, found $270.1136 ;[\alpha]_{\mathrm{D}}{ }^{23}=+96.6\left(\mathrm{c}=0.5, \mathrm{CHCl}_{3}\right)$.

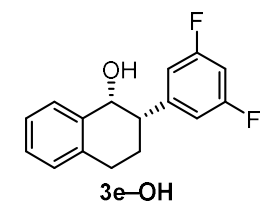

(1R, 2S)-2-(3,5-difluorophenyl)-1,2,3,4-tetrahydronaphthalen-1-ol (3e-OH): The general procedure B was followed using 1,2-dihydronaphthalene (1a, $13 \mathrm{mg}, 0.10 \mathrm{mmol})$, Ligand $((\boldsymbol{R})$-L12, $10 \mathrm{mg}, 0.01 \mathrm{mmol})$ and 4-nitrophenyldiazonium tetrafluoroborate (2e, $45 \mathrm{mg}, 0.20$ mmol). Purification of this material by chromatography on silica gel (EtOAc : petroleum ether $=$ $1: 10)$ afforded product $\mathbf{3 e -}-\mathbf{O H}$ as a yellow solid $(7 \mathrm{mg}, 28 \%$ yield, $>20: 1 \mathrm{dr}, 94: 6 \mathrm{er}): \mathrm{Rf}=0.4$ $($ EtOAc : petroleum ether $=1: 5) ;{ }^{1} \mathbf{H}$ NMR $\left(\mathrm{CDCl}_{3}, 400 \mathrm{MHz}\right) \delta: 7.34-7.18(\mathrm{~m}, 4 \mathrm{H}), 6.88(\mathrm{~d}, J=$ 8.0 Hz, 2H), 6.74-6.69 (m, 1H), $4.79(\mathrm{~s}, 1 \mathrm{H}), 3.07-3.01(\mathrm{~m}, 2 \mathrm{H}), 2.96-2.87(\mathrm{~m}, 1 \mathrm{H}), 2.43-2.33(\mathrm{~m}$, 1H), 1.95-1.93 (m, 1H), 1.55 (s 1H); ${ }^{13} \mathbf{C}$ NMR $\left(\mathrm{CDCl}_{3}, 100 \mathrm{MHz}\right) \delta: 164.4,164.2,161.9,161.8$, 
147.1, 147.0, 146.9, 137.4, 136.3, 130.2, 129.2, 128.4, 126.4, 111.2, 111.1, 111.0, 110.96, 102.3, 102.1, 101.8, 71.0, 45.8, 29.3, $21.6 \mathrm{ppm}$; HRMS (ESI-TOF) $\mathrm{m} / \mathrm{z}$ calcd for $\mathrm{C}_{16} \mathrm{H}_{13} \mathrm{OF}_{2}(\mathrm{M}-\mathrm{H})^{-}$: 259.0934 , found259.0922; $[\alpha]_{\mathrm{D}}^{23}=+109.6\left(\mathrm{c}=0.7, \mathrm{CHCl}_{3}\right)$.

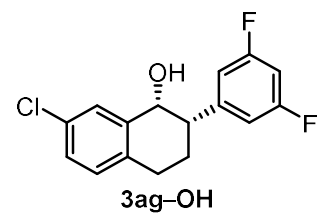

(1R, 2S)-7-chloro-2-(3,5-difluorophenyl)-1,2,3,4-tetrahydronaphthalen-1-ol (3ag-OH): The general procedure B was followed using 6-chloro-1,2-dihydronaphthalene (1c, $17 \mathrm{mg}, 0.10$ mmol) , Ligand ( $(\boldsymbol{R})-\mathbf{L 1 2}, 10 \mathrm{mg}, 0.01 \mathrm{mmol})$ and 4-nitrophenyldiazonium tetrafluoroborate (2e, $45 \mathrm{mg}, 0.20 \mathrm{mmol}$ ). Purification of this material by chromatography on silica gel (EtOAc : petroleum ether $=1: 10)$ afforded product 3ag-OH as a white solid $(6 \mathrm{mg}, 21 \%$ yield, $>20: 1 \mathrm{dr}$, 94:6 er): $\mathrm{Rf}=0.4($ EtOAc $:$ petroleum ether $=1: 5) ;{ }^{1} \mathbf{H} \mathbf{~ N M R}\left(\mathrm{CDCl}_{3}, 400 \mathrm{MHz}\right) \delta: 7.31(\mathrm{~s}, 1 \mathrm{H})$, $7.24(\mathrm{~d}, J=8.0 \mathrm{~Hz}, 1 \mathrm{H}), 7.12(\mathrm{~d}, J=8.0 \mathrm{~Hz}, 1 \mathrm{H}), 6.87(\mathrm{~d}, J=7.7 \mathrm{~Hz}, 2 \mathrm{H}), 6.75-6.71(\mathrm{~m}, 1 \mathrm{H})$, $4.73(\mathrm{~s}, 1 \mathrm{H}), 3.05-2.97(\mathrm{~m}, 2 \mathrm{H}), 2.89-2.81(\mathrm{~m}, 1 \mathrm{H}), 2.40-2.31(\mathrm{~m}, 1 \mathrm{H}), 1.95-1.93(\mathrm{~m}, 1 \mathrm{H}), 1.63(\mathrm{~s}$ $1 \mathrm{H}) ;{ }^{13} \mathrm{C}$ NMR $\left(\mathrm{CDCl}_{3}, 100 \mathrm{MHz}\right) \delta: 164.4,164.3,162.0,161.8,146.5,146.4,146.3,139.0,134.7$, $131.8,130.5,129.9,128.5,111.22,111.15,111.04,110.97,102.6,102.3,102.0,70.6,45.6,28.8$, $21.5 \mathrm{ppm}$; HRMS (ESI-TOF) $\mathrm{m} / z$ calcd for $\mathrm{C}_{16} \mathrm{H}_{12} \mathrm{BOF}_{2} \mathrm{Cl}(\mathrm{M}-\mathrm{H}): 293.0545$, found 293.0536; $[\alpha]_{\mathrm{D}}^{23}=+35.2\left(\mathrm{c}=0.6, \mathrm{CHCl}_{3}\right)$.

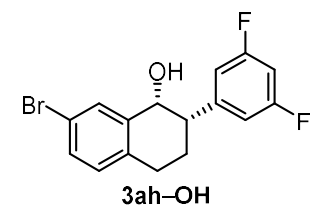

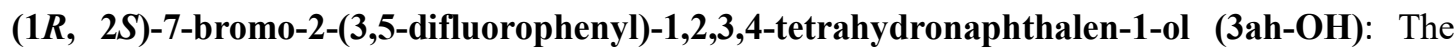
general procedure $\mathrm{B}$ was followed using 6-bromo-1,2-dihydronaphthalene (1d, $21 \mathrm{mg}, 0.10$ $\mathrm{mmol})$, Ligand ((R)-L12,10 $\mathrm{mg}, 0.01 \mathrm{mmol})$ and 4-nitrophenyldiazonium tetrafluoroborate (2e, $45 \mathrm{mg}, 0.20 \mathrm{mmol}$ ). Purification of this material by chromatography on silica gel (EtOAc : petroleum ether $=1: 10)$ afforded product $\mathbf{3 a h}-\mathbf{O H}$ as a yellow solid $(9 \mathrm{mg}, 27 \%$ yield, $>20: 1 \mathrm{dr}$, 95:5 er): $\mathrm{Rf}=0.4($ EtOAc $:$ petroleum ether $=1: 5) ;{ }^{1} \mathbf{H} \mathbf{~ N M R ~}\left(\mathrm{CDCl}_{3}, 400 \mathrm{MHz}\right) \delta: 7.46(\mathrm{~s}, 1 \mathrm{H})$, $7.38(\mathrm{~d}, J=8.0 \mathrm{~Hz}, 1 \mathrm{H}), 7.06(\mathrm{~d}, J=8.0 \mathrm{~Hz}, 1 \mathrm{H}), 6.86(\mathrm{~d}, J=7.7 \mathrm{~Hz}, 2 \mathrm{H}), 6.75-6.71(\mathrm{~m}, 1 \mathrm{H})$, $4.72(\mathrm{~s}, 1 \mathrm{H}), 3.04-2.95(\mathrm{~m}, 2 \mathrm{H}), 2.87-2.78(\mathrm{~m}, 1 \mathrm{H}), 2.40-2.291(\mathrm{~m}, 1 \mathrm{H}), 1.95-1.92(\mathrm{~m}, 1 \mathrm{H}), 1.64(\mathrm{~s}$ $1 \mathrm{H}) ;{ }^{13} \mathrm{C}$ NMR $\left(\mathrm{CDCl}_{3}, 100 \mathrm{MHz}\right) \delta: 164.4,164.3,161.9,161.8,146.4,146.33,146.25,139.4$, 135.2, 132.9, 131.4, 130.8, 119.6, 111.21, 111.15, 111.03, 110.96, 102.6, 102.3, 102.1, 70.5, 45.6, 28.8, 21.4 ppm; HRMS (ESI-TOF) $\mathrm{m} / z$ calcd for $\mathrm{C}_{16} \mathrm{H}_{12} \mathrm{BOF}_{2} \mathrm{Br}(\mathrm{M}-\mathrm{H}$ ): 337.0040 , found $337.0055 ;[\alpha]_{\mathrm{D}}^{23}=+63.4\left(\mathrm{c}=0.9, \mathrm{CHCl}_{3}\right)$. 


\section{References}

1. Wedek, V.; Lommel, R. V.; Daniliuc, C. G.; Proft, F. D.; Hennecke, U. Angew. Chem. Int. Ed. 2019, 58,9239 .

2. Sokolov, A.; Kozlinsky, S. G. I.; Dolia, K.; Tolmachev, A. A.; Kuchkovska, Y.; Grygorenko, O. O. Synthesis 2019, 51, 848-858

3. Kong, D.; Han, S.; Zi, G.; Hou, G.; Zhang, J. J. Org. Chem. 2018, 83, 1924.

4. Dong, D.-J.; Li, Y.; Wang, J.-Q.; Tian, S.-K. Chem. Commun., 2011, 47, 2158.

5. Kuroda, J.; Inamoto, K.; Hiroya, K.; Doi, T. Eur. J. Org. Chem. 2009, 2251.

6. Denmark, S. E.; Regens, C. S. Tetrahedron Letters 2011, 52, 2165.

6. Chen, P.-P.; Lucas, E. L.; Greene, M. A.; Zhang, S.-Q.; Tollefson, E. J.; Erickson, L. W.; Taylor, B. L. H.; Jarvo, E. R.; Hong, X. J. Am. Chem. Soc. 2019, 141, 5835.

7. Haydl, A. M.; Hartwig, J. F. Org. Lett. 2019, 21, 1337.

8. Lundgren, R. J.; Bryce N. Thomas, Chem. Commun. 2016, 52, 958.

9. Hoque, I. U.; Chowdhury, S. R.; Maity, S. J. Org. Chem. 2019, 84, 3025. 


\section{The data for some failed examples}

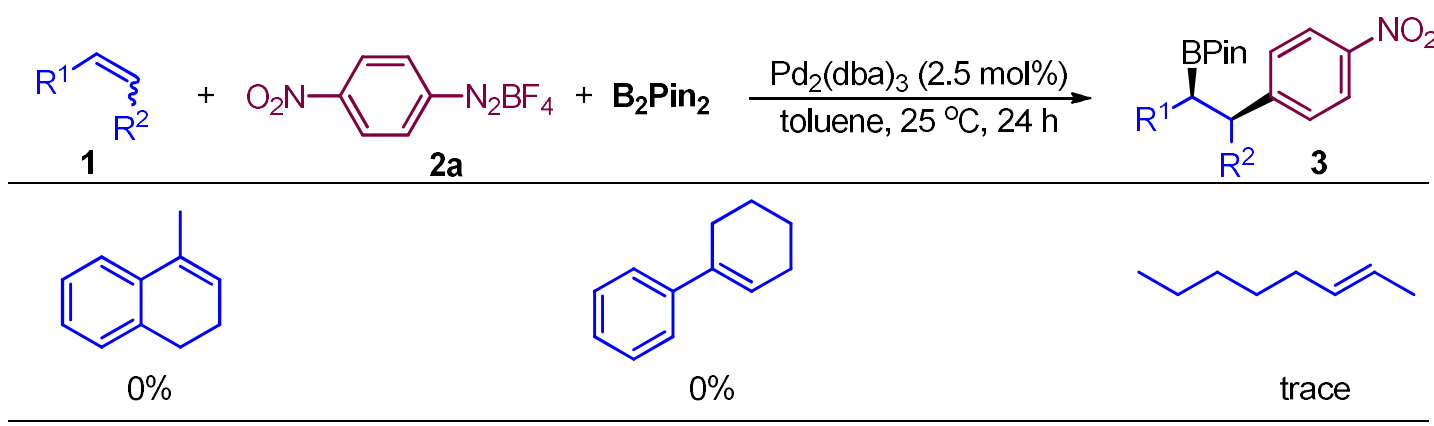

a Standard reaction conditions: 1 ( $0.2 \mathrm{mmol}), 2 \mathrm{a}(0.3 \mathrm{mmol}), \mathrm{B}_{2} \mathrm{pin}_{2}(0.3 \mathrm{mmol}), \mathrm{Pd}_{2}(\mathrm{dba})_{3}(2.5 \mathrm{~mol} \%)$, toluene ( $2 \mathrm{~mL}), \mathrm{N}_{2}, 25^{\circ} \mathrm{C}, 24 \mathrm{~h}$. 


\section{The data for $1 \mathrm{mmol}$ scale reaction}

$\mathrm{R}^{1 \overbrace{\mathrm{R}^{2}}}+\mathrm{ArN}_{2} \mathrm{BF}_{4}+\mathrm{B}_{2} \mathrm{Pin}_{2} \underset{\text { toluene, } 25^{\circ} \mathrm{C}, 24 \mathrm{~h}}{\stackrel{\mathrm{Pd}_{2}(\mathrm{dba})_{3}(2.5 \mathrm{~mol} \%)}{\longrightarrow}}$ 1

2<smiles>[R]C([AlH2])[C@@H]([R2])[BiH]</smiles>

3

The general procedure for $1 \mathrm{mmol}$ scale reaction: In an oven dried $50 \mathrm{~mL}$ Schlenk tube containing a stirring bar was charged with charged with $\mathrm{B}_{2} \mathrm{Pin}_{2}(1.5 \mathrm{mmol}), \mathrm{Pd}_{2} \mathrm{dba}_{3}(2.5 \mathrm{~mol} \%)$, and $\mathrm{ArN}_{2} \mathrm{BF}_{4}(1.5 \mathrm{mmol})$. The tube was then evacuated and back-filled under a $\mathrm{N}_{2}$ flow (this sequence was repeated three times). Anhydrous toluene $(10.0 \mathrm{ml})$, alkene 1 (1.0 mmol ) were added subsequently under $\mathrm{N}_{2}$. The tube was stirred at $25{ }^{\circ} \mathrm{C}$ for $24 \mathrm{~h}$. The reaction was concentrated by rotary evaporation. The residue was purified by silica gel column chromatography (EtOAc/petroleum ether) to afford the product 3.

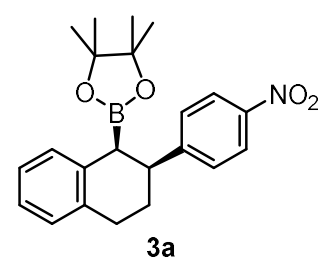

4,4,5,5-tetramethyl-2-(2-(4-nitrophenyl)-1,2,3,4-tetrahydronaphthalen-1-yl)-1,3,2-dioxaborol ane (3a): The general procedure for $1 \mathrm{mmol}$ scale reaction was followed using 1,2-dihydronaphthalene (1a, $130 \mathrm{mg}, 1.0 \mathrm{mmol})$ and 4-nitrophenyldiazonium tetrafluoroborate (2a $356 \mathrm{mg}, 1.5 \mathrm{mmol}$ ). Purification of this material by chromatography on silica gel (EtOAc : petroleum ether $=1: 20)$ afforded product $\mathbf{3 a}$ as a colorless solid (281 $\mathrm{mg}, 74 \%$ yield, $>20: 1 \mathrm{dr})$ : $\mathrm{Rf}=0.6($ EtOAc $:$ petroleum ether $=1: 10)$.

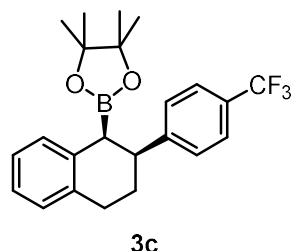

4,4,5,5-tetramethyl-2-(2-(4-(trifluoromethyl)phenyl)-1,2,3,4-tetrahydronaphthalen-1-yl)-1,3,2 -dioxaborolane (3c): The general procedure for $1 \mathrm{mmol}$ scale reaction was followed using 1,2-dihydronaphthalene (1a, $130 \mathrm{mg}, 1.0 \mathrm{mmol}$ ) and 4-(trifluoromethyl)phenyl diazonium tetrafluoroborate $(\mathbf{2 c}, 390 \mathrm{mg}, 1.5 \mathrm{mmol})$. Purification of this material by chromatography on silica gel (EtOAc : petroleum ether $=1: 40$ ) afforded product $\mathbf{3 c}$ as a colorless solid $(346 \mathrm{mg}, 86 \%$ yield, $>20: 1 \mathrm{dr}): \mathrm{Rf}=0.8($ EtOAc $:$ petroleum ether $=1: 10)$. 
9. Result for X-ray Analysis<smiles>Fc1ccc([C@@H]2CCc3ccccc3[C@@H]2[B]c2ccccc2)cc1</smiles>

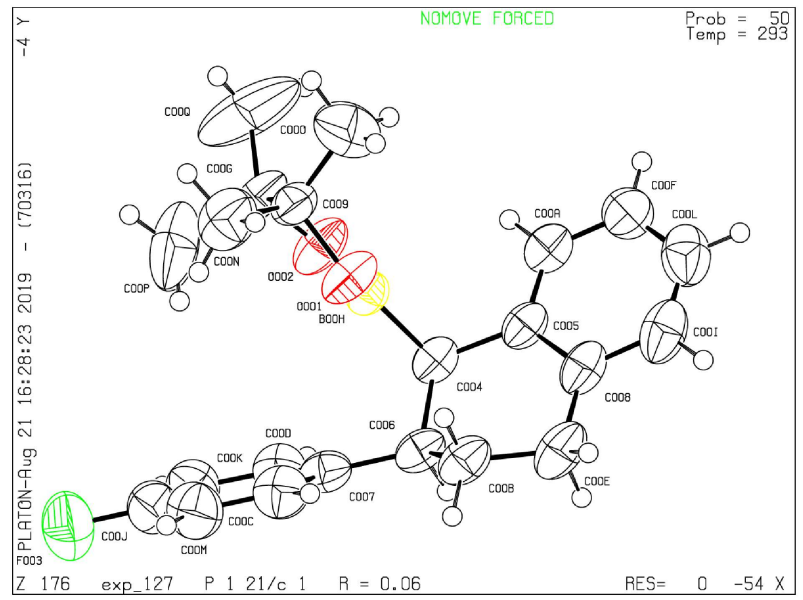

Figure 8.1: X-ray result of $\mathbf{3 f}$
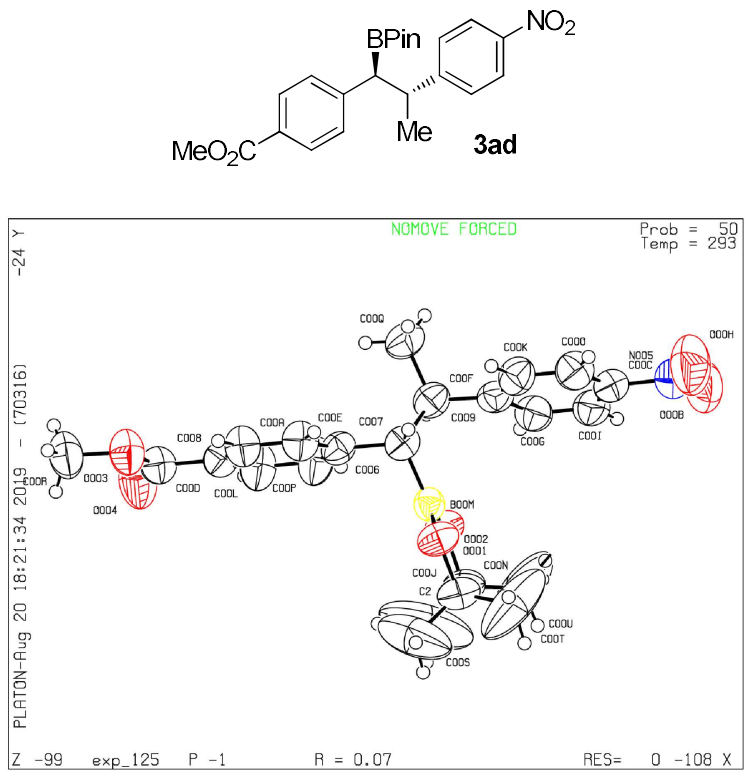

Figure 8.1: X-ray result of 3ad

Conclusion: The X-ray data of $3 f$ and 3ad crystal could support the syn-selectivity of this Pd-catalyzed reaction. 


\section{Determination of diastereoselectivity}

Using 3a-OH as object, the signal of the other isomer could not be found in ${ }^{1} \mathrm{H}-\mathrm{NMR}$. So we proposed the d.r. higher than 20:1. To double make sure d.r. of this chemistry, we separated and show all the diastereomers of $\mathbf{3 a - O H}$ in the HPLC traces. The detail data was shown at bellow. These data suggested the diastereoselectivity of this reaction is more than 20:1. So HPLC result supported the validity of the conclusion from $1 \mathrm{H}-\mathrm{NMR}$ data.

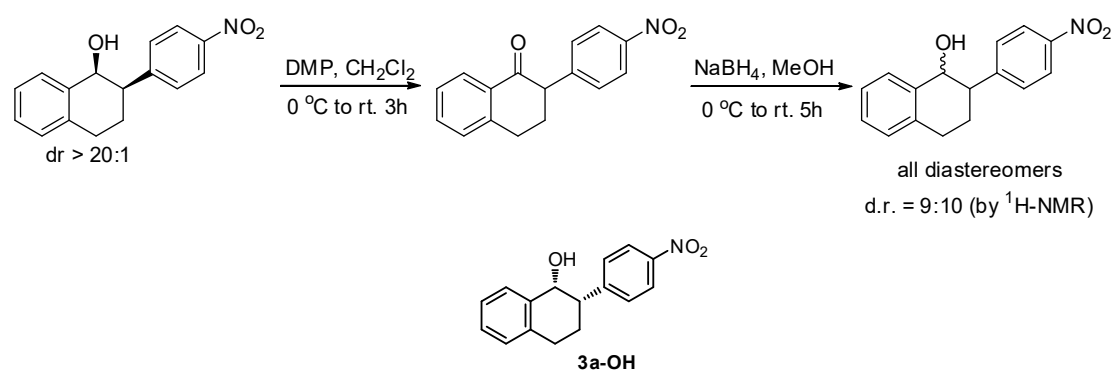

Separation of enantiomers by HPLC, Daicel Chiralpak AD-H column, $20{ }^{\circ} \mathrm{C}$, Hexane : $i-\mathrm{PrOH}=$

$90: 10,1 \mathrm{~mL} / \mathrm{min}$, major retention time: $\mathrm{t}_{1}=22.6$, minor retention time: $\mathrm{t}_{2}=35.7$; er $=93: 7$.

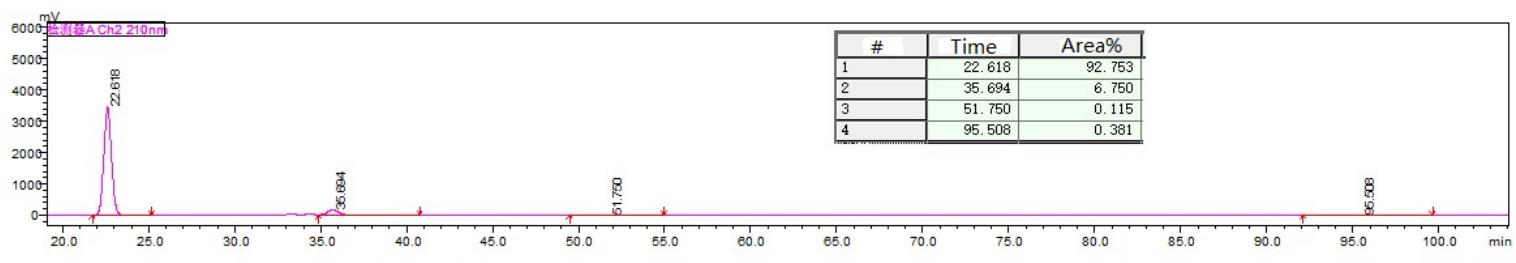

3a-OH

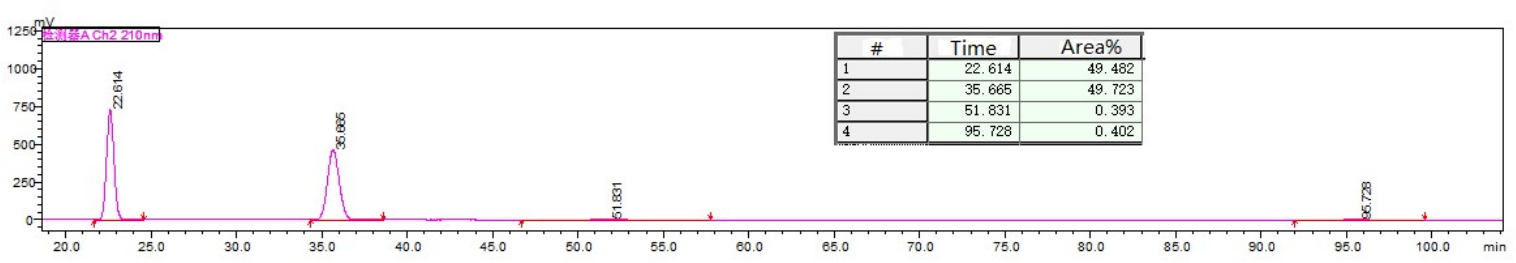

Rac-3a-OH

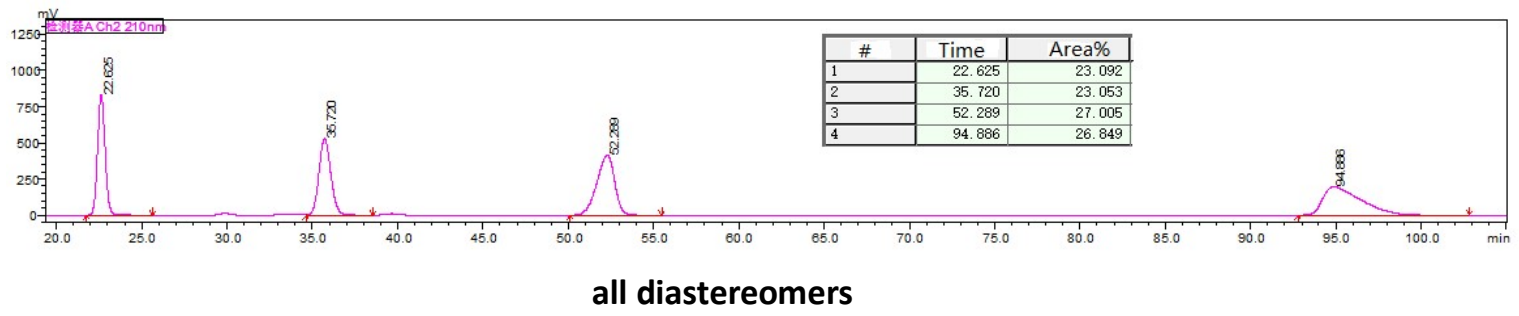




\section{The absolute configuration of the products:}

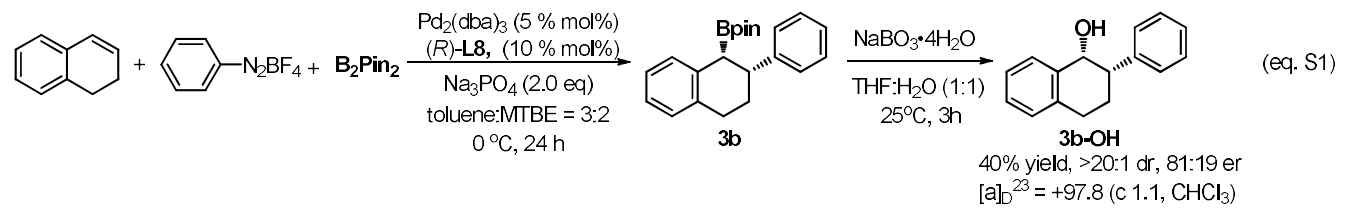

The $\mathbf{3 b} \mathbf{b}-\mathbf{O H}$ could be made by present reaction (eq. S1). The data of $\mathbf{3 b - O H}$ as bellow:

$(1 R, 2 S)$-2-phenyl-1,2,3,4-tetrahydronaphthalen-1-ol (3b-OH): The general procedure B was followed using 1,2-dihydronaphthalene (1a, $13 \mathrm{mg}, 0.10 \mathrm{mmol})$, Ligand ((R)-L8, $6 \mathrm{mg}, 0.01 \mathrm{mmol})$ and 4-nitrophenyldiazonium tetrafluoroborate $(\mathbf{2 b}, 39 \mathrm{mg}, 0.20 \mathrm{mmol})$. Purification of this 11 material by chromatography on silica gel (EtOAc : petroleum ether $=1: 10)$ afforded product $\mathbf{3 b}-\mathbf{O H}$ as a White solid $(9 \mathrm{mg}, 40 \%$ yield, $>20: 1 \mathrm{dr}, 81: 19 \mathrm{er}): \mathrm{Rf}=0.4$ (EtOAc : petroleum ether $=1: 5)$; ${ }^{1} \mathbf{H}$ NMR $\left(\mathrm{CDCl}_{3}, 400 \mathrm{MHz}\right) \delta: 7.40-7.33(\mathrm{~m}, 5 \mathrm{H}), 7.30-7.18(\mathrm{~m}, 4 \mathrm{H}), 4.79(\mathrm{~s}$, $1 \mathrm{H}), 3.13-3.02(\mathrm{~m}, 2 \mathrm{H}), 2.96-2.88(\mathrm{~m}, 1 \mathrm{H}), 2.51-2.40(\mathrm{~m}, 1 \mathrm{H}), 1.98-1.95(\mathrm{~m}, 1 \mathrm{H}), 1.57(\mathrm{~s}, 1 \mathrm{H}) ;{ }^{13} \mathbf{C ~ N M R}\left(\mathrm{CDCl}_{3}\right.$, $100 \mathrm{MHz}) \delta: 142.6,137.6,136.7,130.4,129.1,128.6,128.2,128.1,126.8,126.1,71.3,46.0,29.7,21.5$ ppm; $[\alpha]_{\mathrm{D}}^{23}=+97.8\left(\mathrm{c}=1.1, \mathrm{CHCl}_{3}\right)$.

Separation of enantiomers by HPLC, Daicel Chiralpak AD-H column, $20{ }^{\circ} \mathrm{C}$, Hexane : $i$-PrOH $=90: 10,1 \mathrm{~mL} / \mathrm{min}$, major retention time: $\mathrm{t}_{1}=10.0$, minor retention time: $\mathrm{t}_{2}=18.3$; er $=81: 19$.

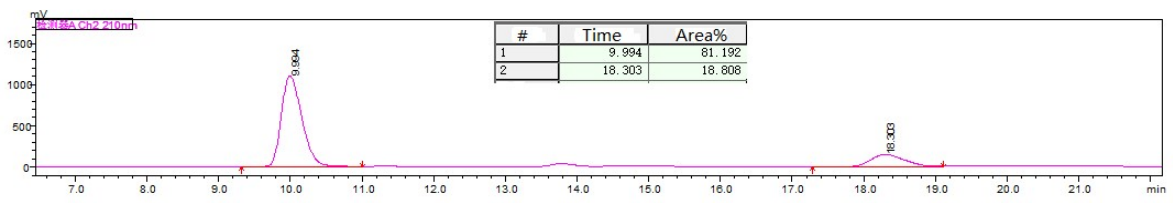

3b-OH product

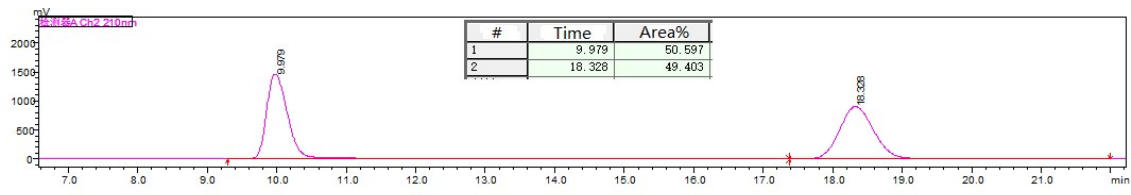

3b-OH rac-sample

Based on the reported ${ }^{1} \mathrm{H}$ NMR, ${ }^{13} \mathrm{C}$ NMR and $[\alpha]_{\mathrm{D}}{ }^{23}$ data data of $\mathbf{3 b}$-OH (reference: Chem. Sci. 2018, 9, 4505), the absolute configuration of $3 \mathbf{b}-\mathbf{O H}$ should $(1 R, 2 S)-2$-phenyl-1,2,3,4tetrahydronaphthalen-1-ol (see as bellow)<smiles>O[C@H]1c2ccccc2CC[C@H]1c1ccc(I)cc1</smiles> 


\section{Enantiomeric ratio of products}

Entry Compound

1<smiles>O=[N+]([O-])c1ccc(C2Cc3ccccc3C2O)cc1</smiles>

2<smiles>Oc1ccccc1C1CCc2ccc(F)cc2[C@H]1O</smiles>

$3 \mathrm{e}-\mathrm{OH}$

3<smiles>OC1c2cc(Cl)ccc2[C@H](O)C1c1cc(F)cc(F)c1</smiles>

4<smiles>O[C@H]1Cc2ccc(Br)cc2[C@H](O)[C@@H]1c1cc(F)cc(F)c1</smiles>

Conditions

HPLC, Column Daicel Chiralpak

AD-H, $20^{\circ} \mathrm{C}$, Hexane $: i-\mathrm{PrOH}=$ $90: 10,1 \mathrm{~mL} / \mathrm{min}$

HPLC, Column Daicel Chiralpak

AD-H, $20^{\circ} \mathrm{C}$, Hexane : $i-\mathrm{PrOH}=95: 5$, $1 \mathrm{~mL} / \mathrm{min}$

HPLC, Column Daicel Chiralpak

$\mathrm{AD}-\mathrm{H}, 20^{\circ} \mathrm{C}$, Hexane $: i-\mathrm{PrOH}=98: 2$, $1 \mathrm{~mL} / \mathrm{min}$

HPLC, Column Daicel Chiralpak

$\mathrm{AD}-\mathrm{H}, 20^{\circ} \mathrm{C}$, Hexane :i-PrOH$=98: 2$, $1 \mathrm{~mL} / \mathrm{min}$
Retention time (min) Er

$\mathrm{t}_{1}=22.6, \mathrm{t}_{2}=35.7$

$\mathrm{t}_{1}=15.6, \mathrm{t}_{2}=19.9 \quad 94: 6$

$\mathrm{t}_{1}=35.4, \mathrm{t}_{2}=78.8$ 94:6

$\mathrm{t}_{1}=34.0, \mathrm{t}_{2}=89.6$ 95:5 


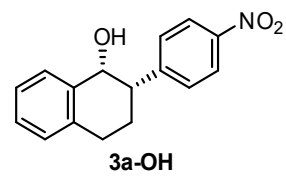

Separation of enantiomers by HPLC, Daicel Chiralpak AD-H column, $20^{\circ} \mathrm{C}$, Hexane : $i-\mathrm{PrOH}=$ $90: 10,1 \mathrm{~mL} / \mathrm{min}$, major retention time: $\mathrm{t}_{1}=22.6$, minor retention time: $\mathrm{t}_{2}=35.7$; er $=93: 7$.

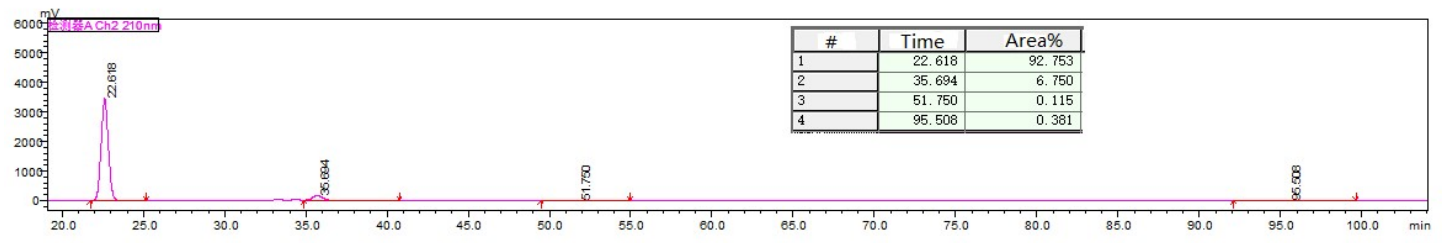

3a-OH product

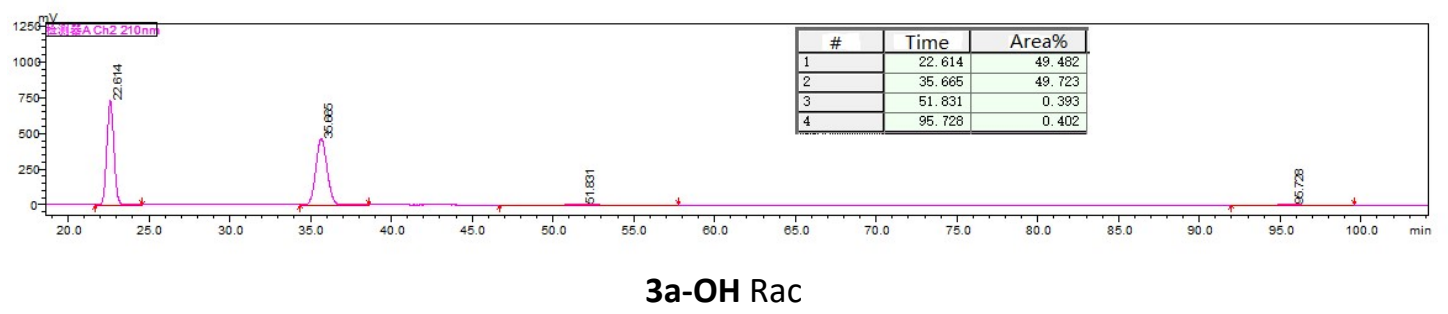




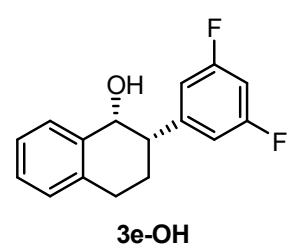

Separation of enantiomers by HPLC, Daicel Chiralpak AD-H column, $20{ }^{\circ} \mathrm{C}$, Hexane : $i-\mathrm{PrOH}=$ $95: 5,1 \mathrm{~mL} / \mathrm{min}$, minor retention time: $\mathrm{t}_{1}=15.6$, major retention time: $\mathrm{t}_{2}=19.9 ; \mathrm{er}=94: 6$
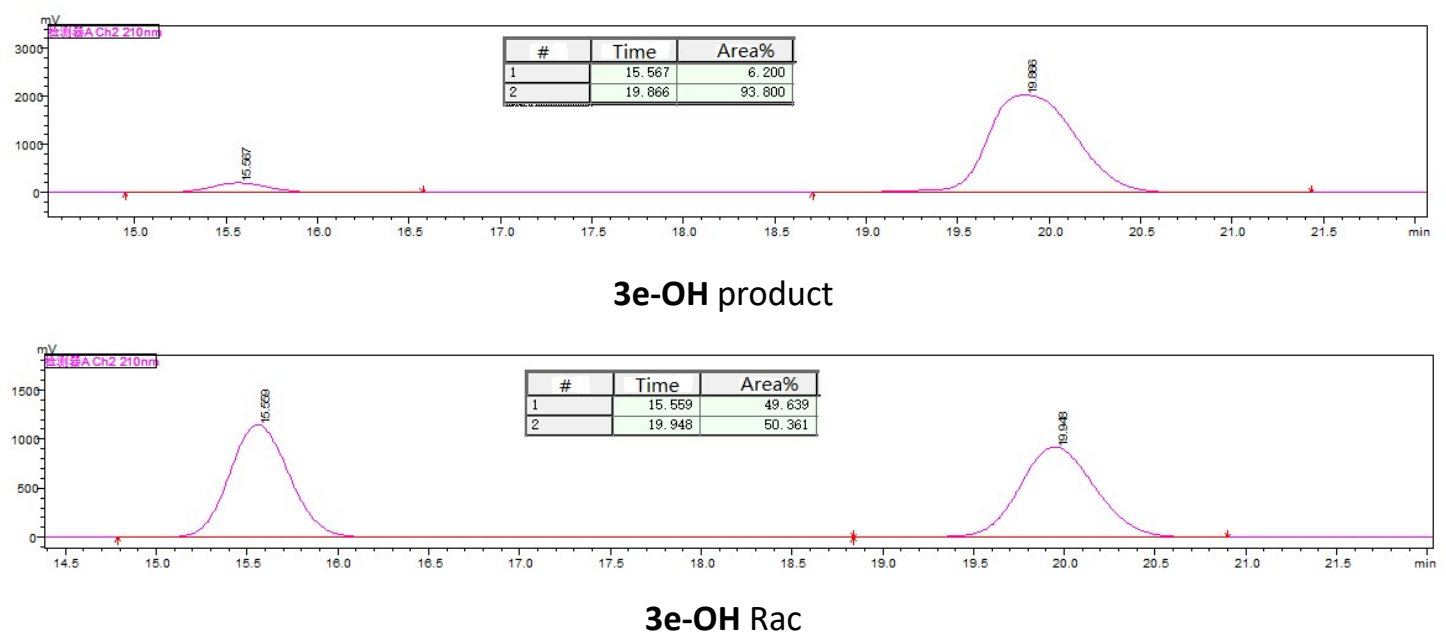


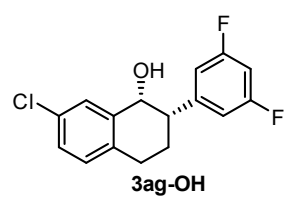

Separation of enantiomers by HPLC, Daicel Chiralpak AD-H column, $20{ }^{\circ} \mathrm{C}$, Hexane : $i-\mathrm{PrOH}=$ $98: 2,1 \mathrm{~mL} / \mathrm{min}$, minor retention time: $\mathrm{t}_{1}=35.4$, major retention time: $\mathrm{t}_{2}=78.8 ; \mathrm{er}=94: 6$

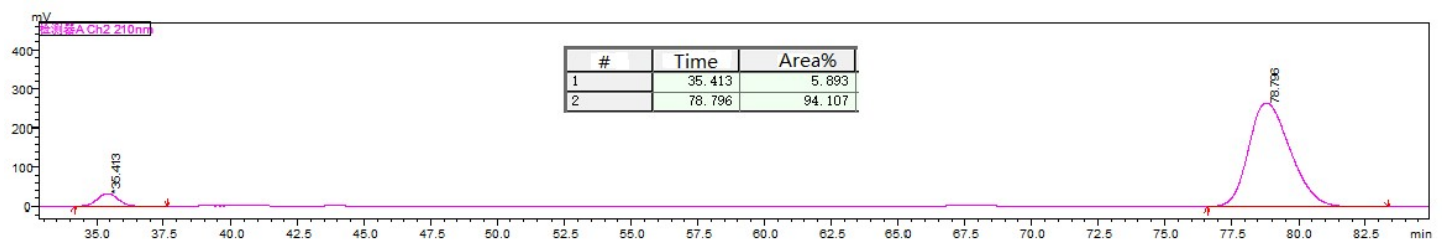

3ag-OH product

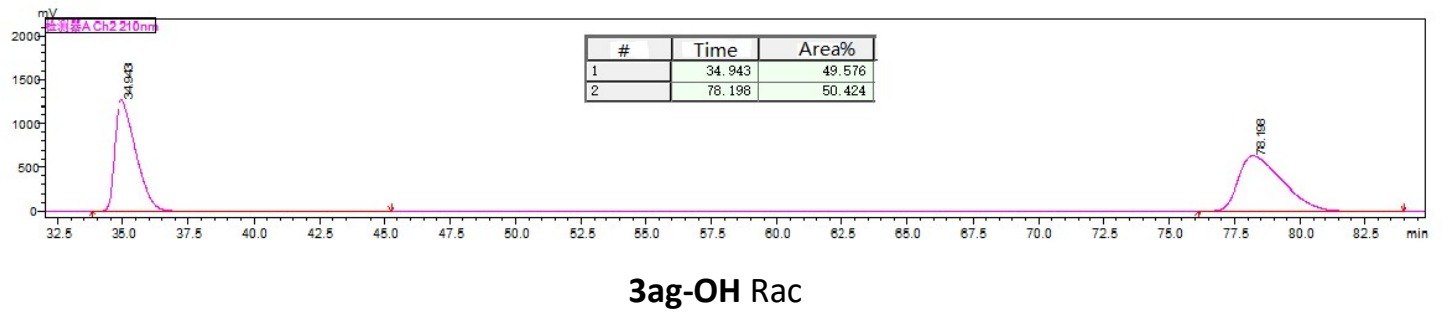




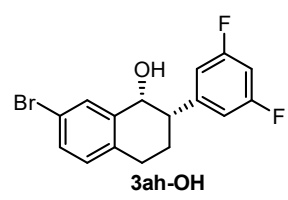

Separation of enantiomers by HPLC, Daicel Chiralpak AD-H column, $20^{\circ} \mathrm{C}$, Hexane : $i-\mathrm{PrOH}=$ $98: 2,1 \mathrm{~mL} / \mathrm{min}$, minor retention time: $\mathrm{t}_{1}=34.0$, major retention time: $\mathrm{t}_{2}=89.6 ; \mathrm{er}=95: 5$

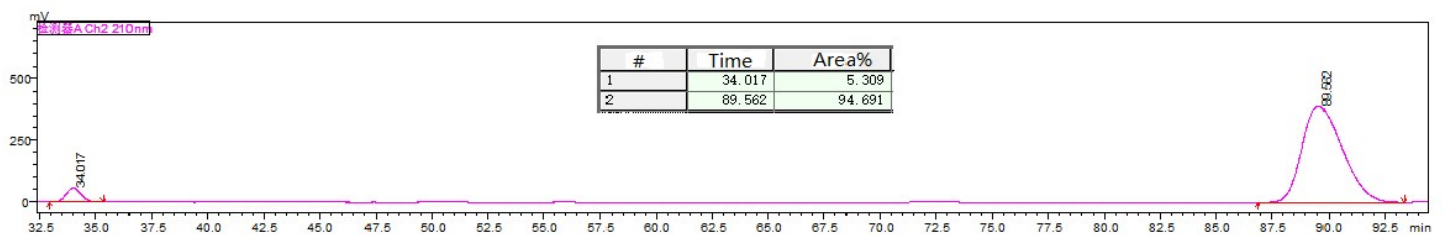

3ah-OH product

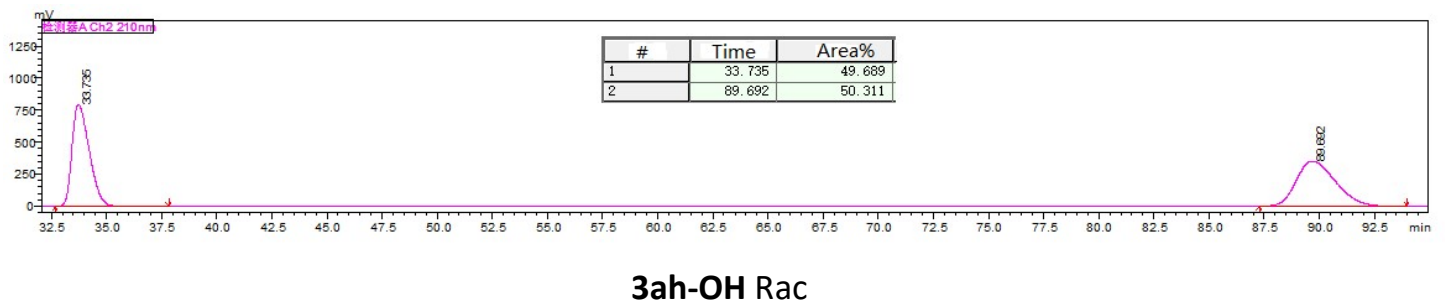




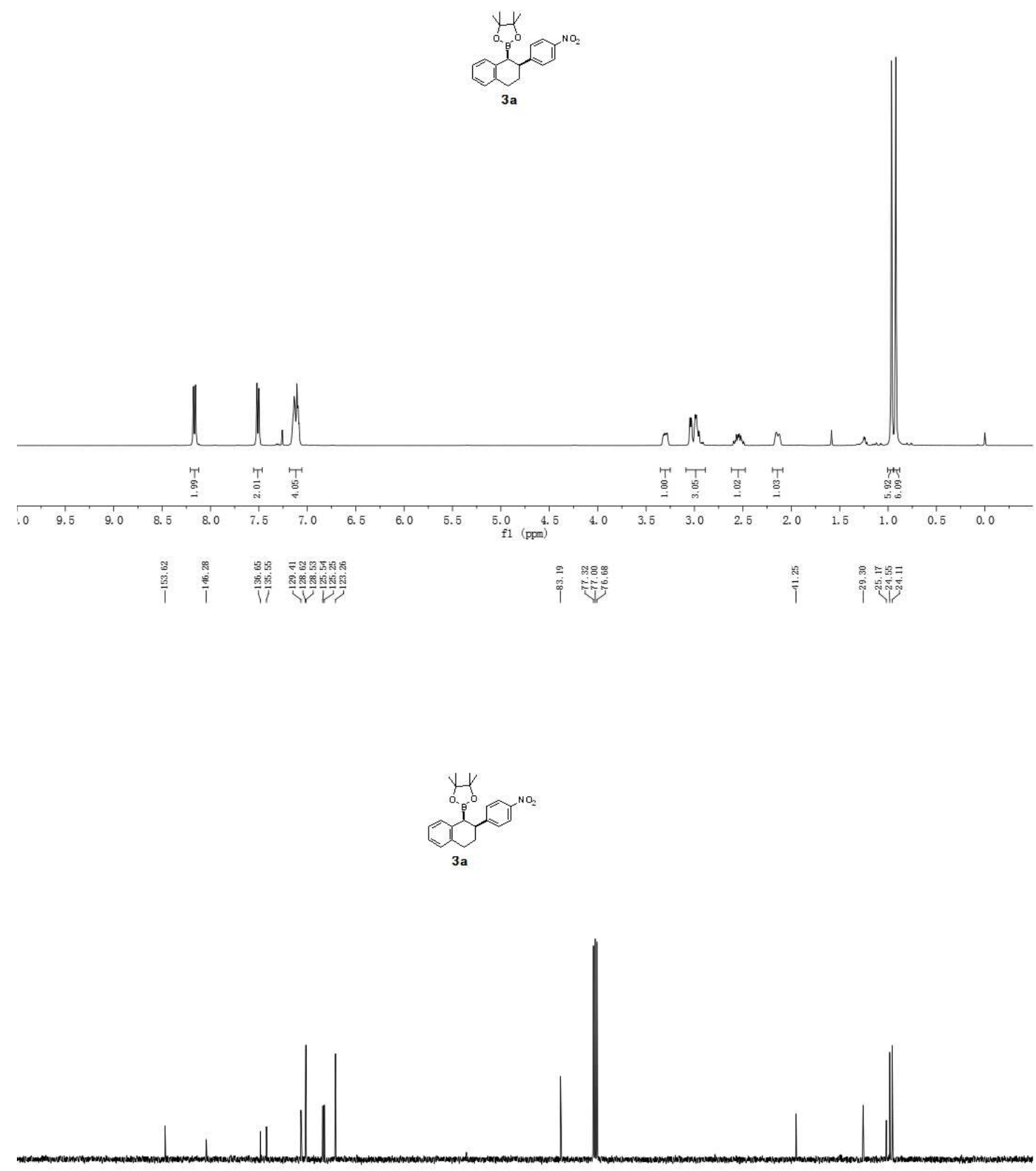

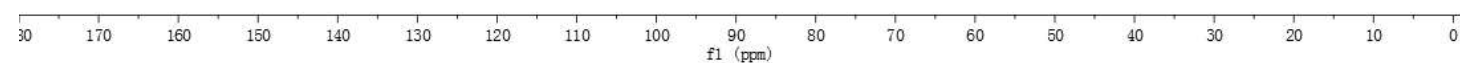



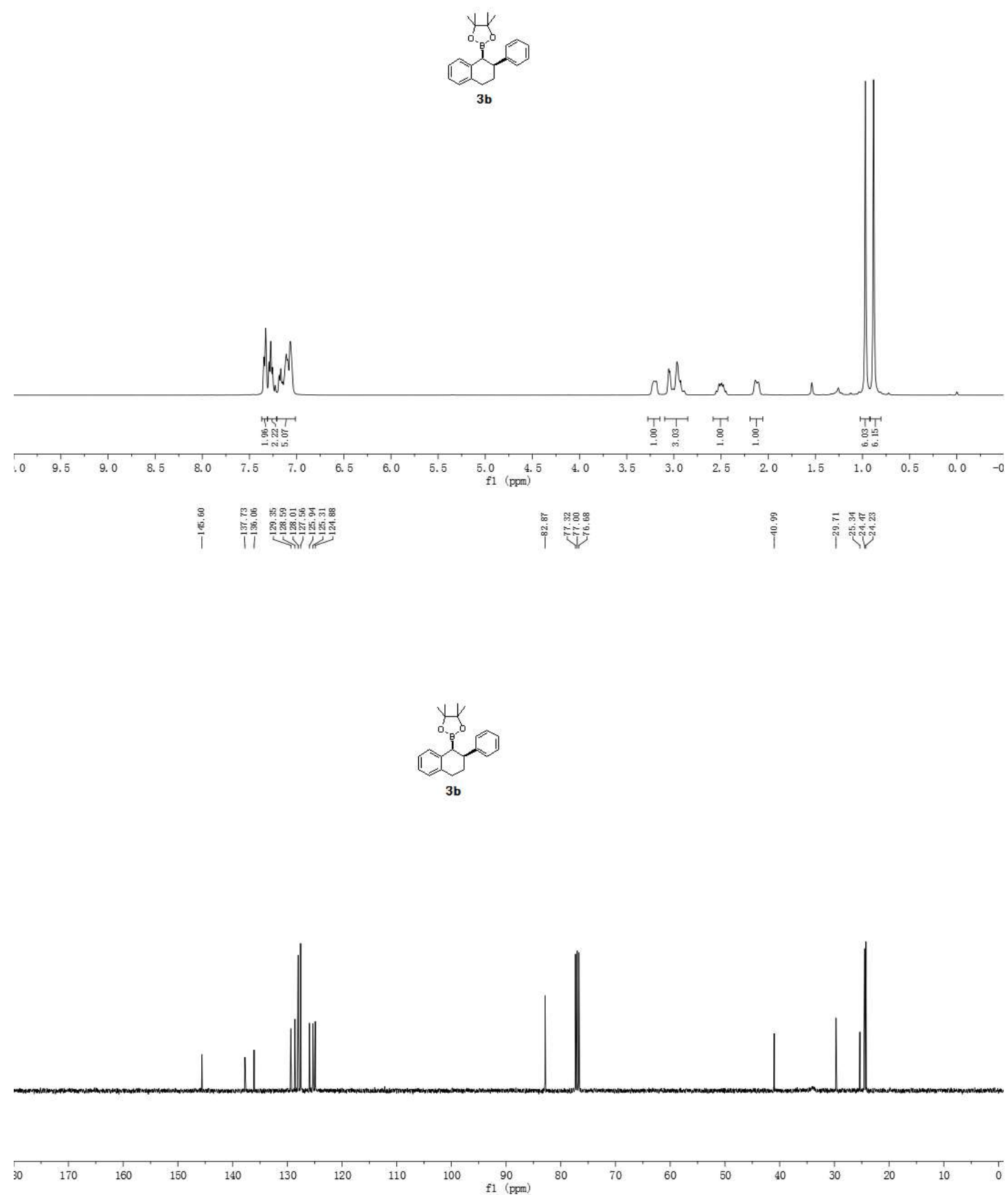


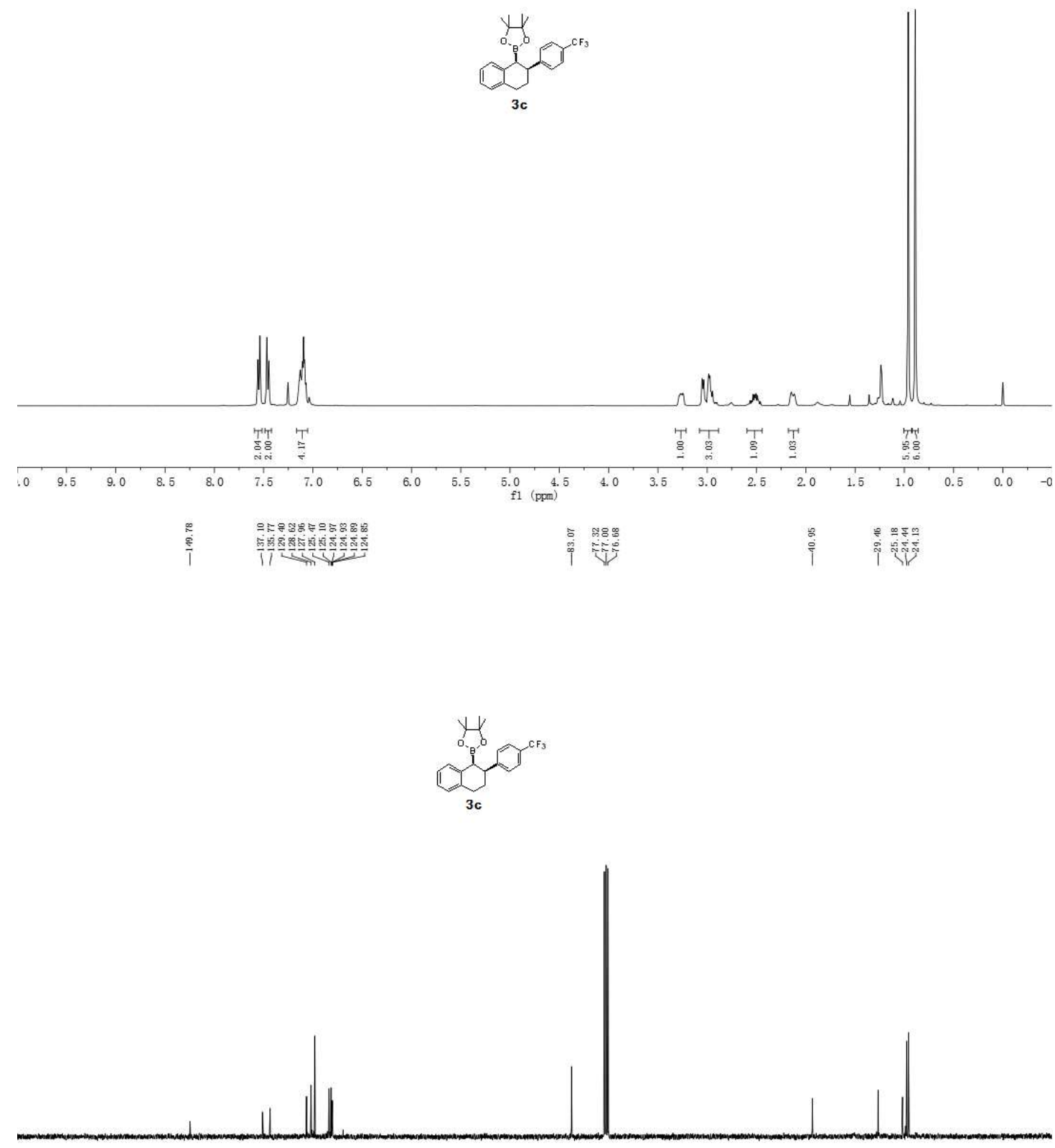

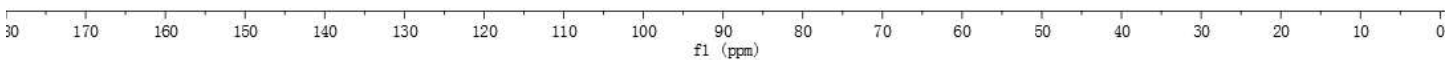




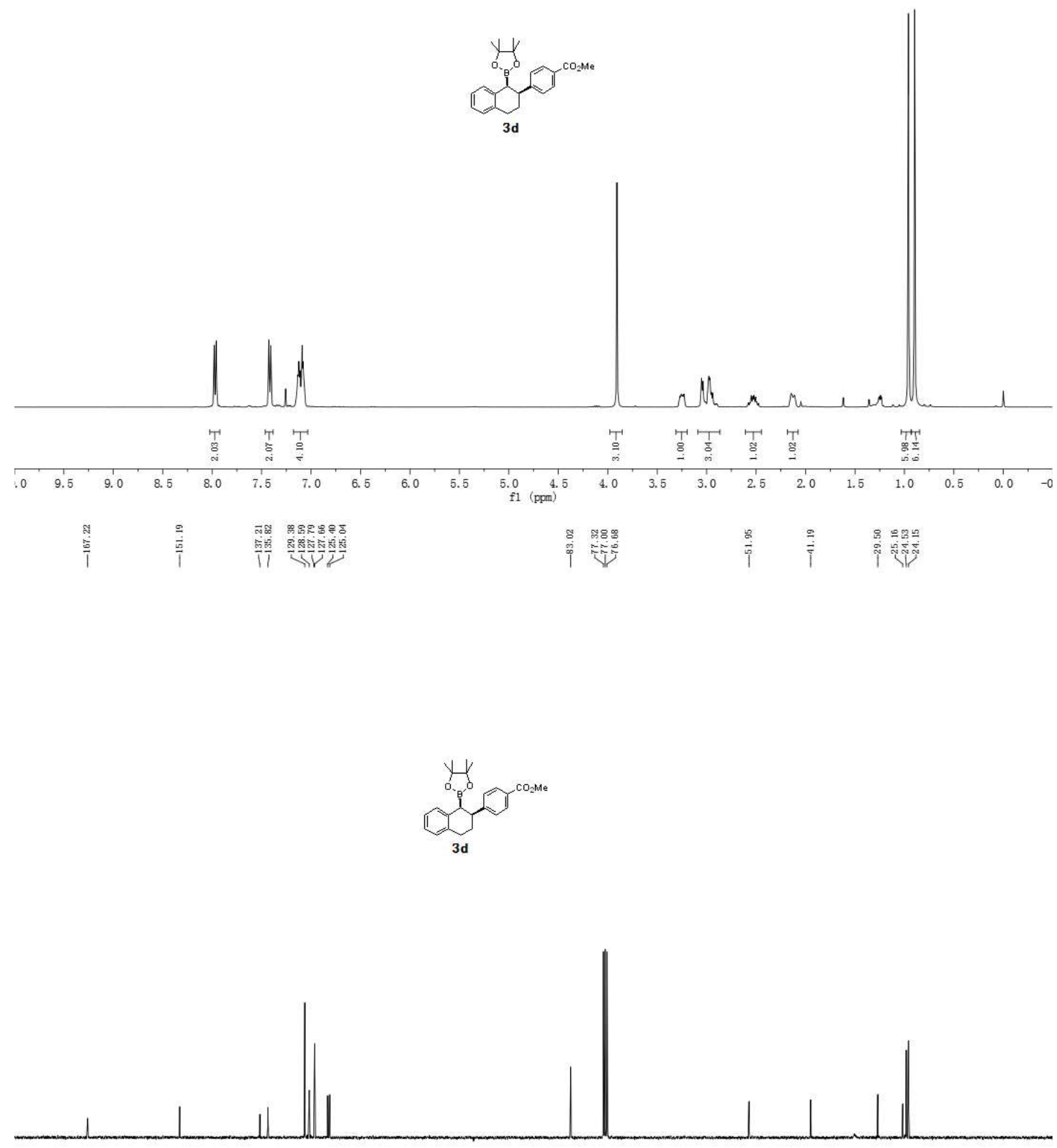

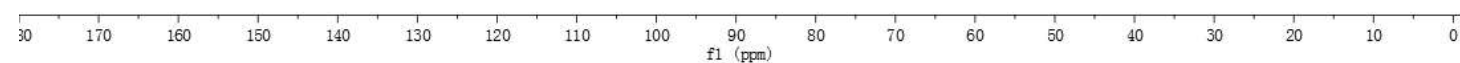



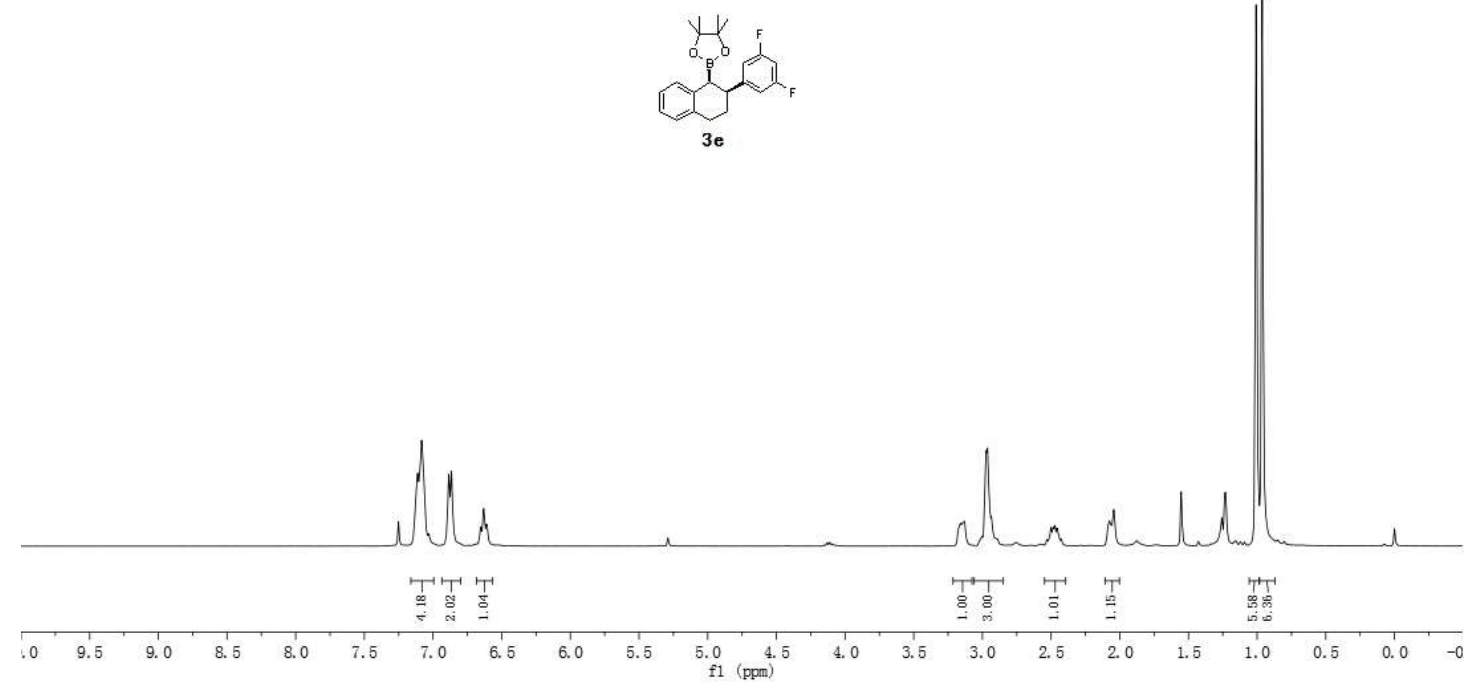

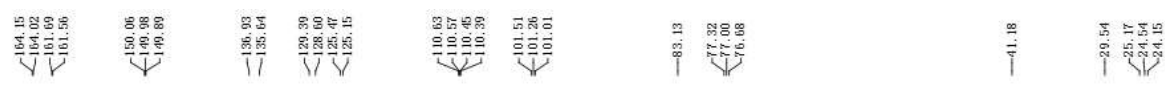
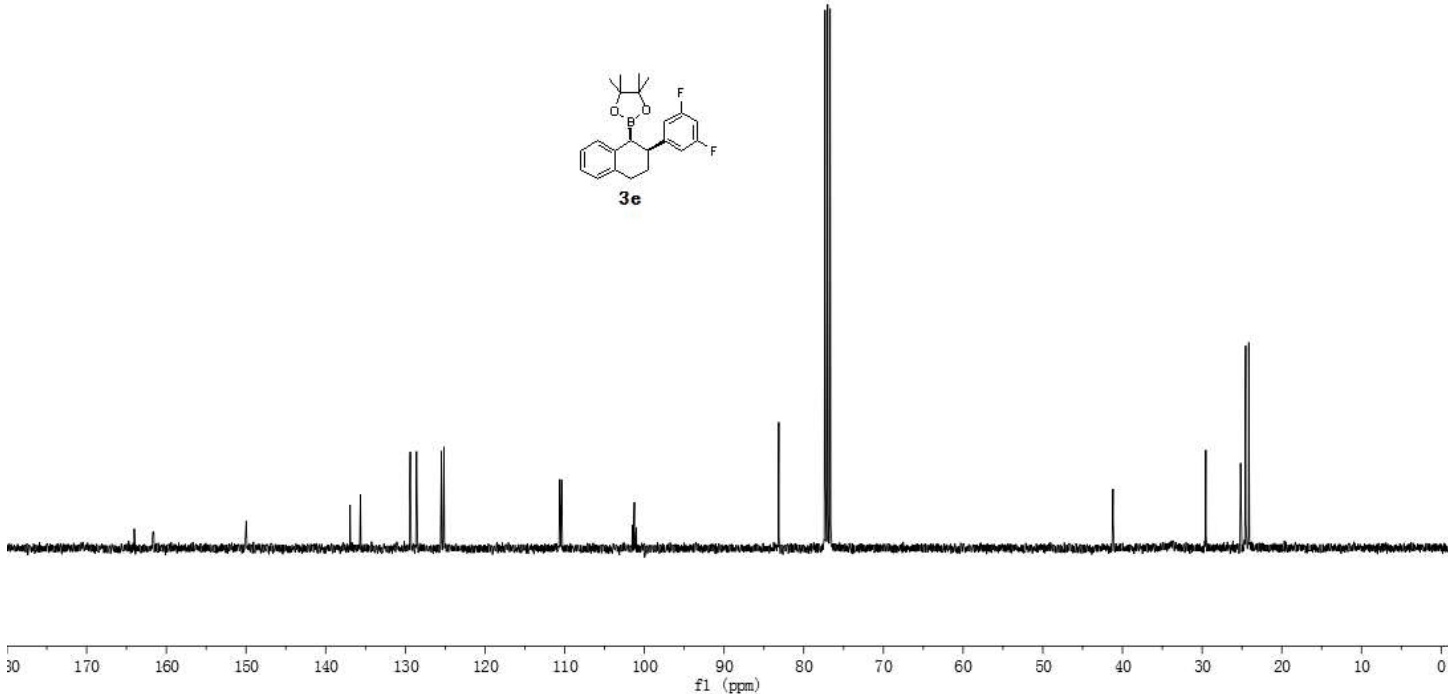

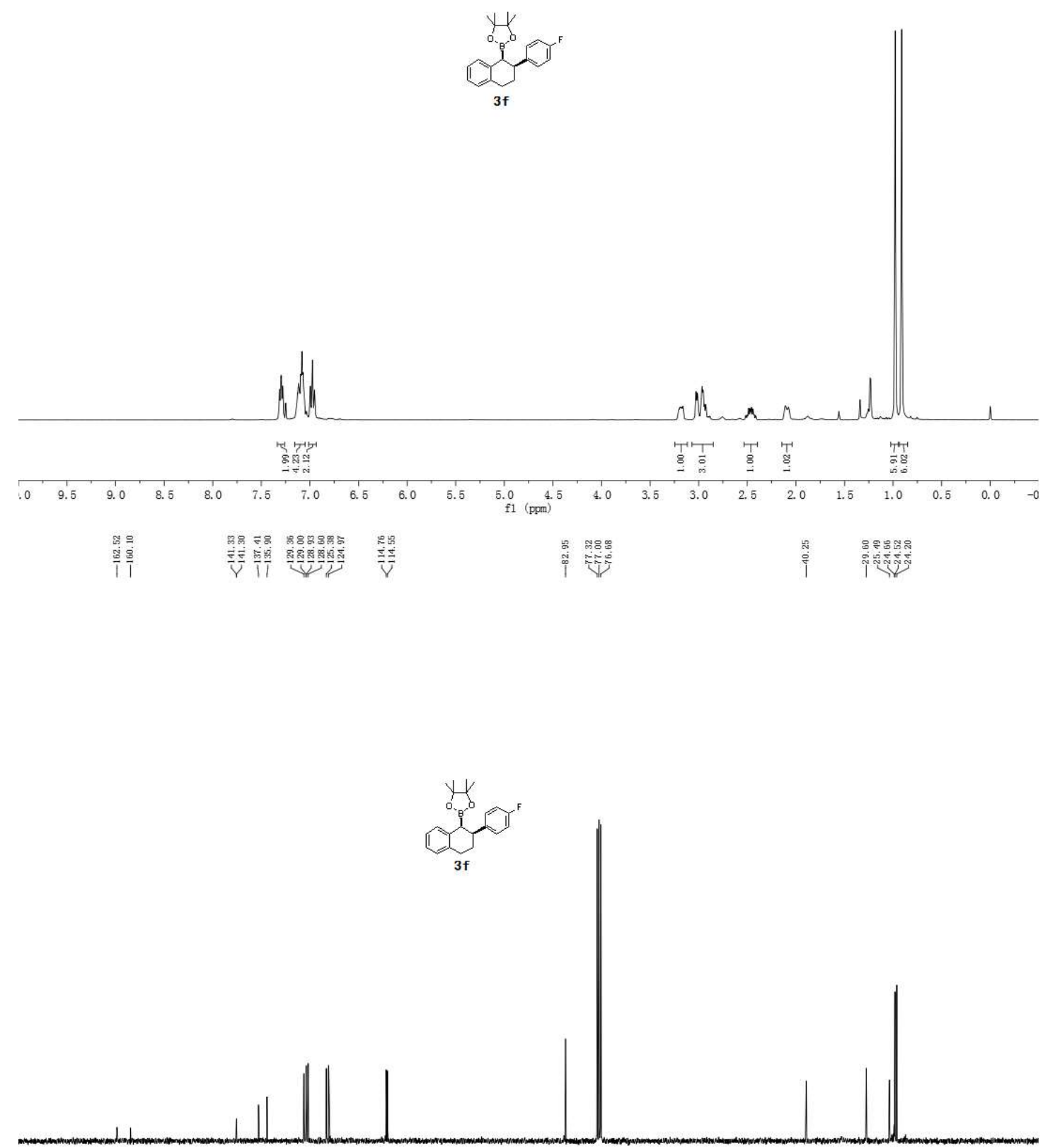

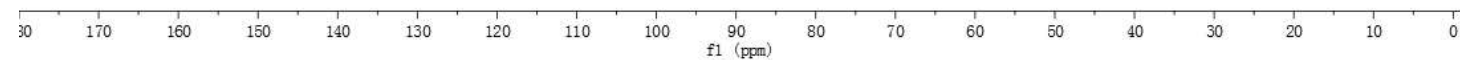



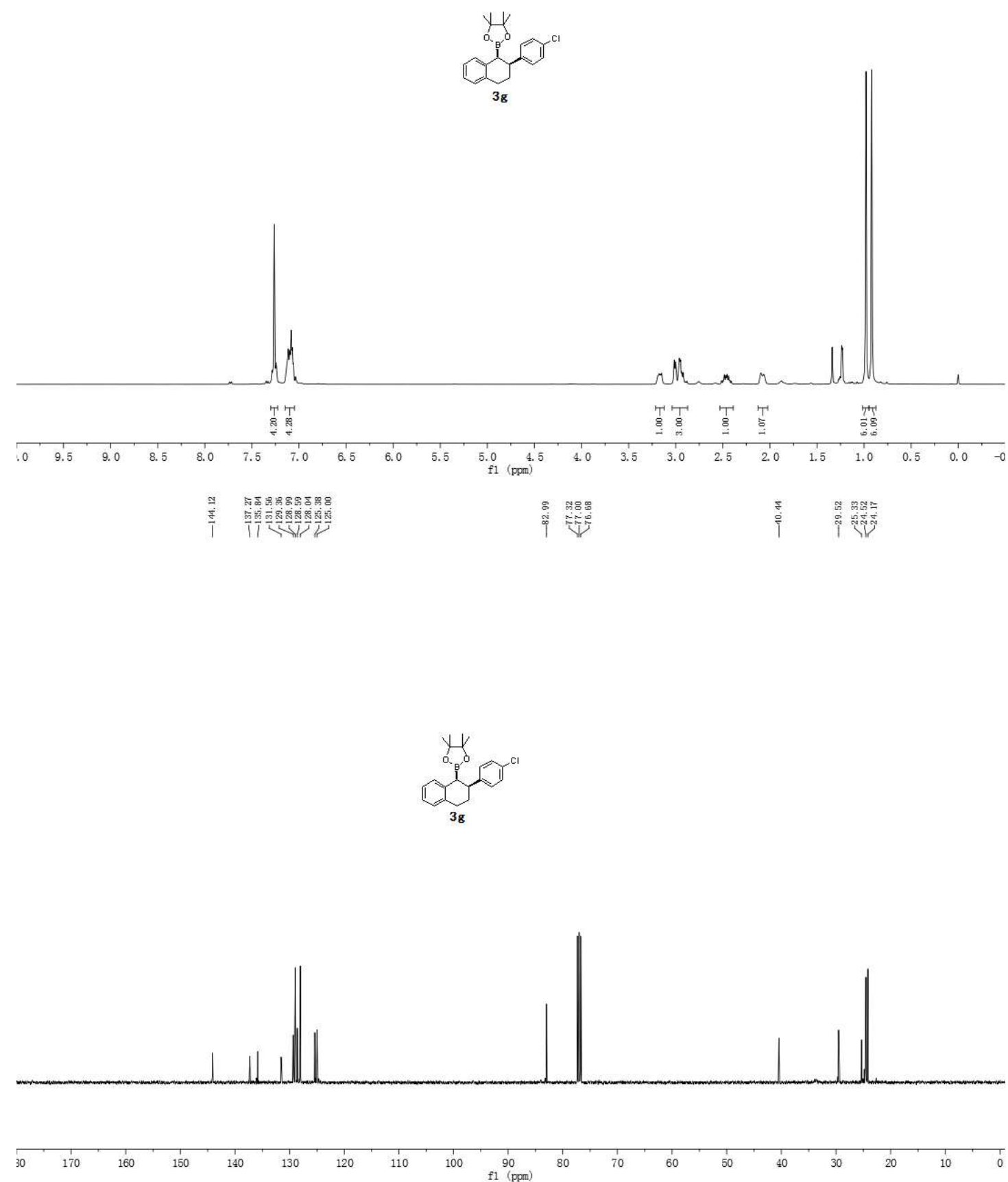

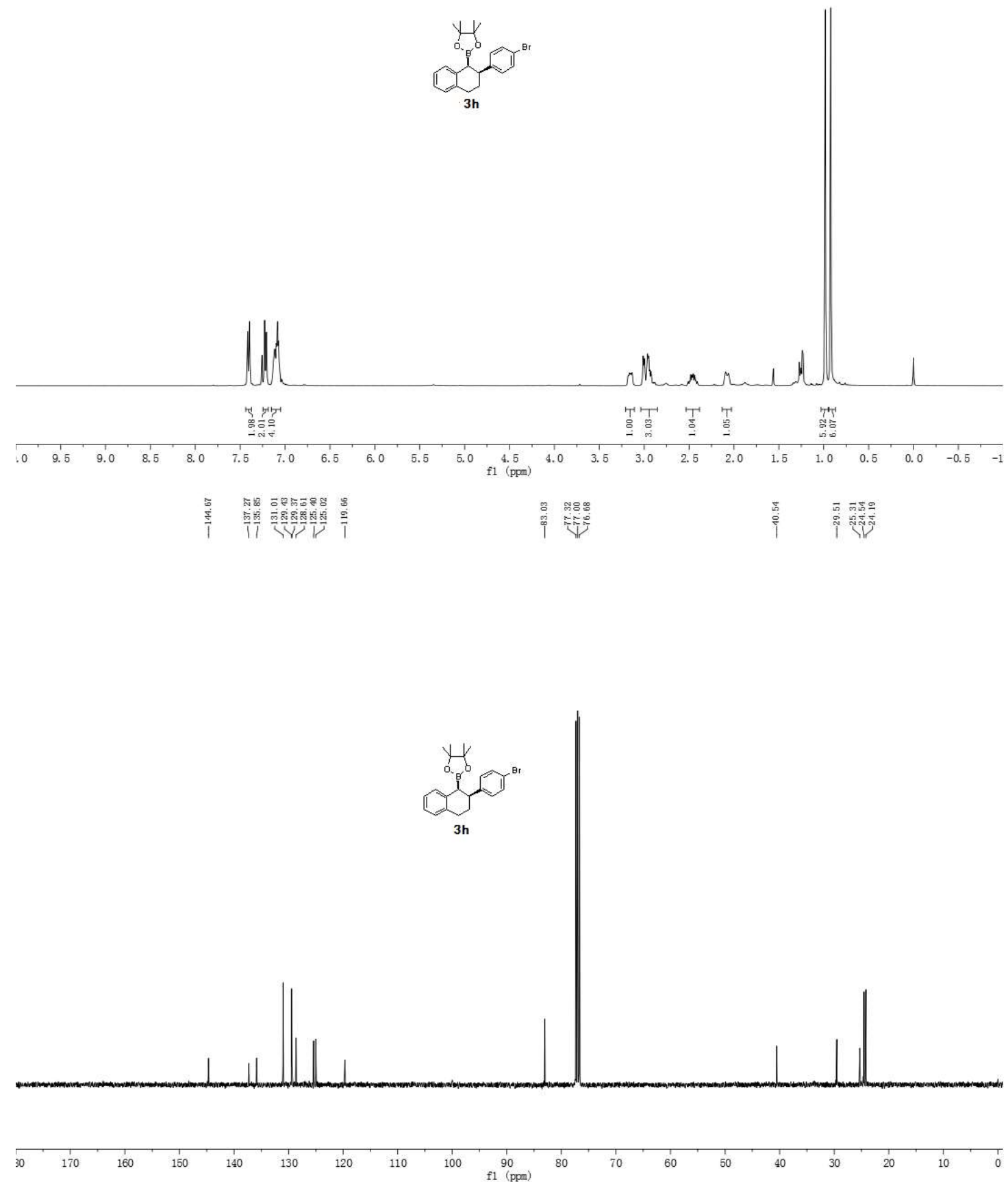


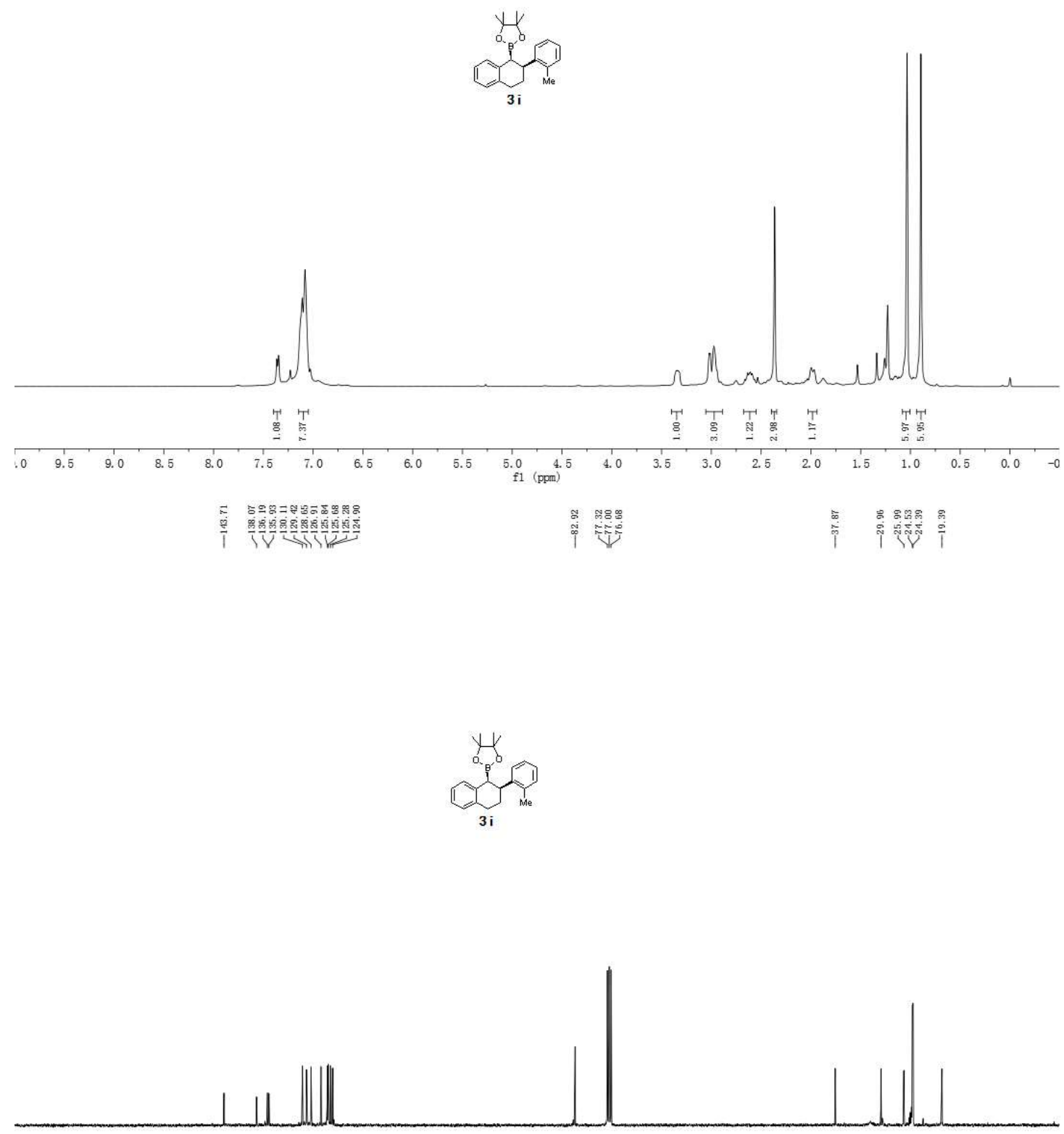

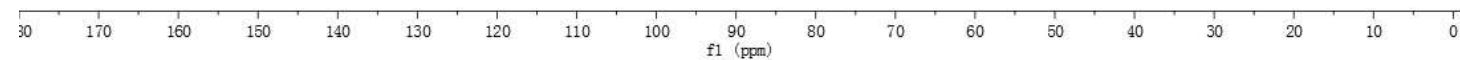



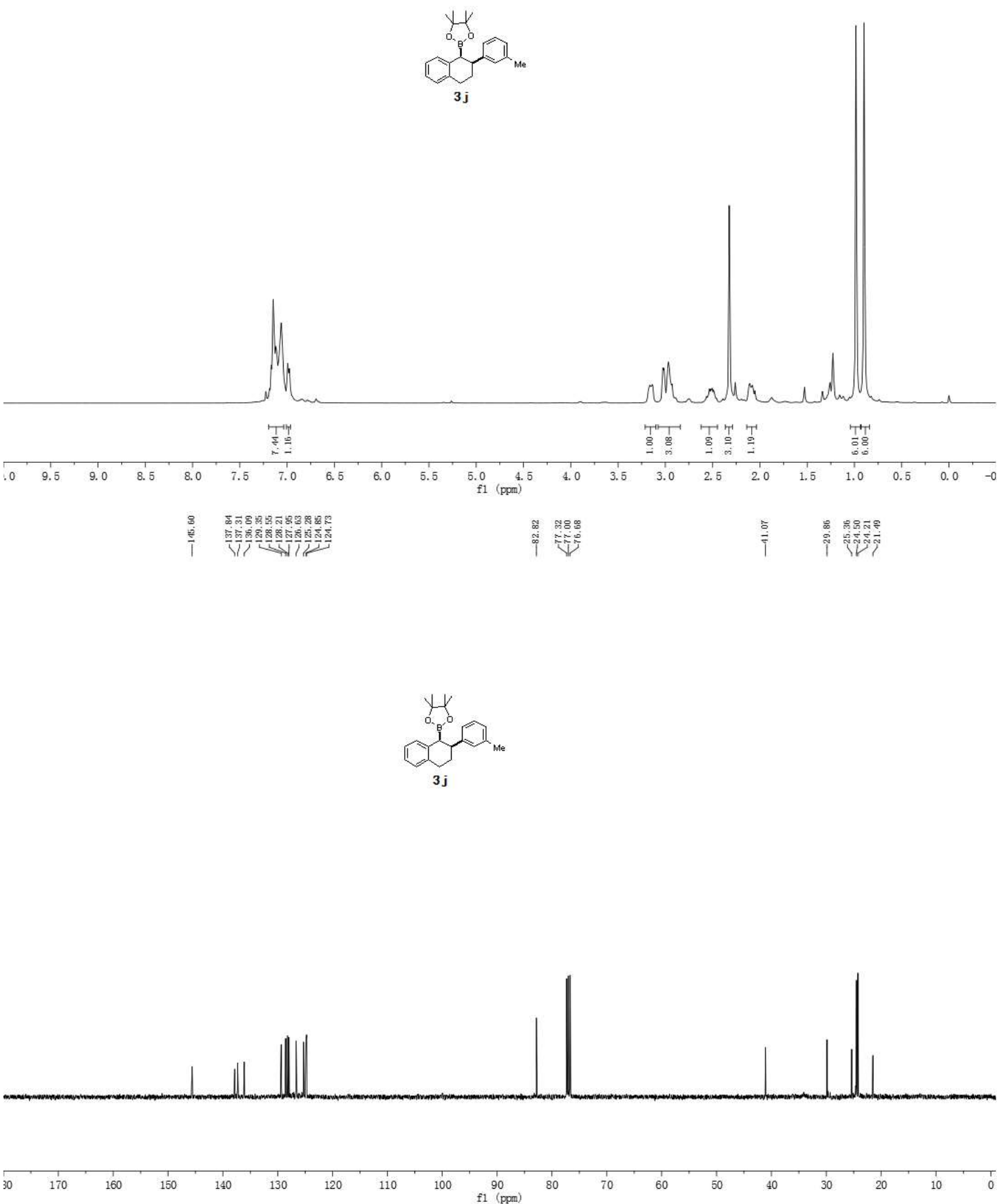

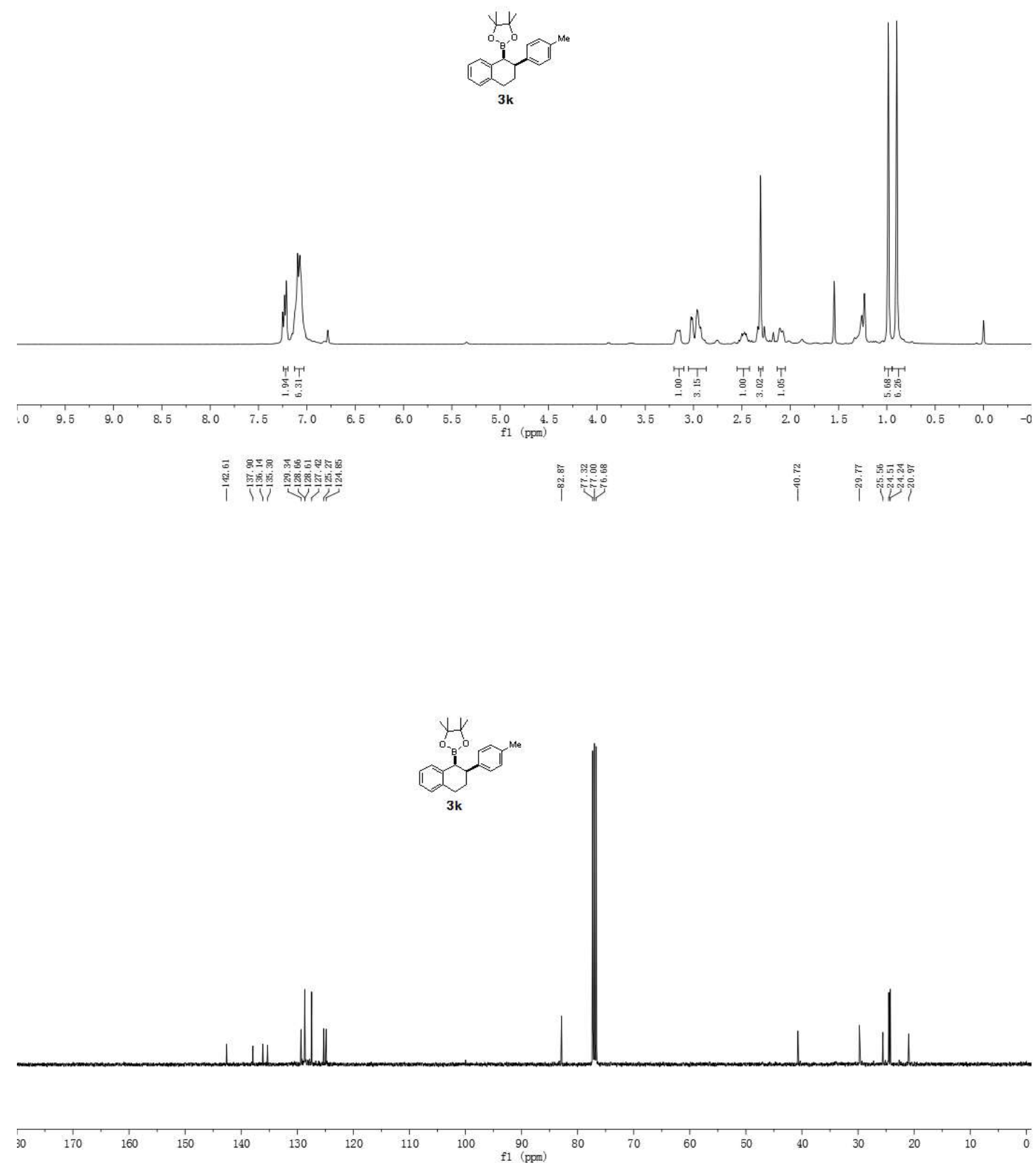

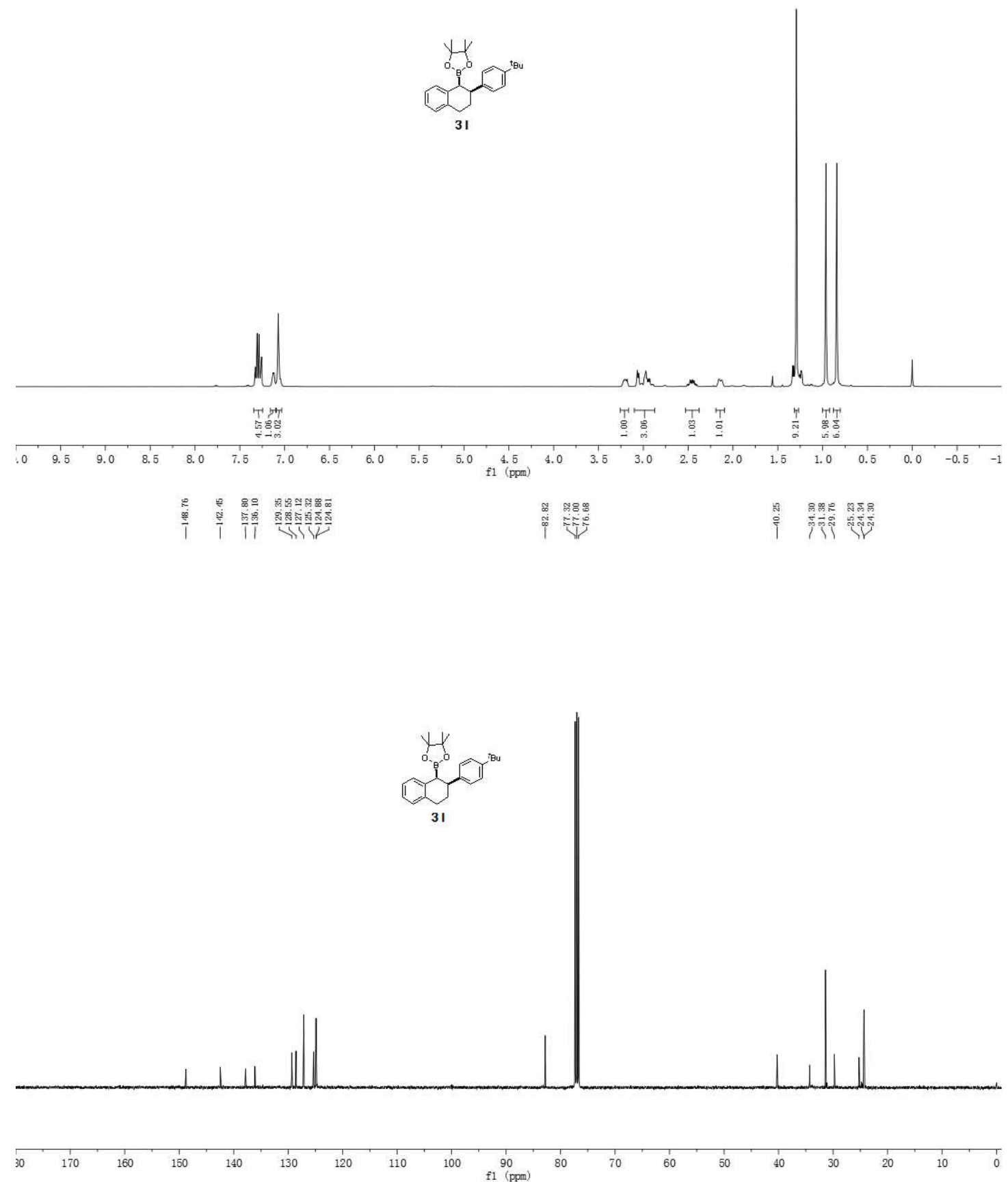


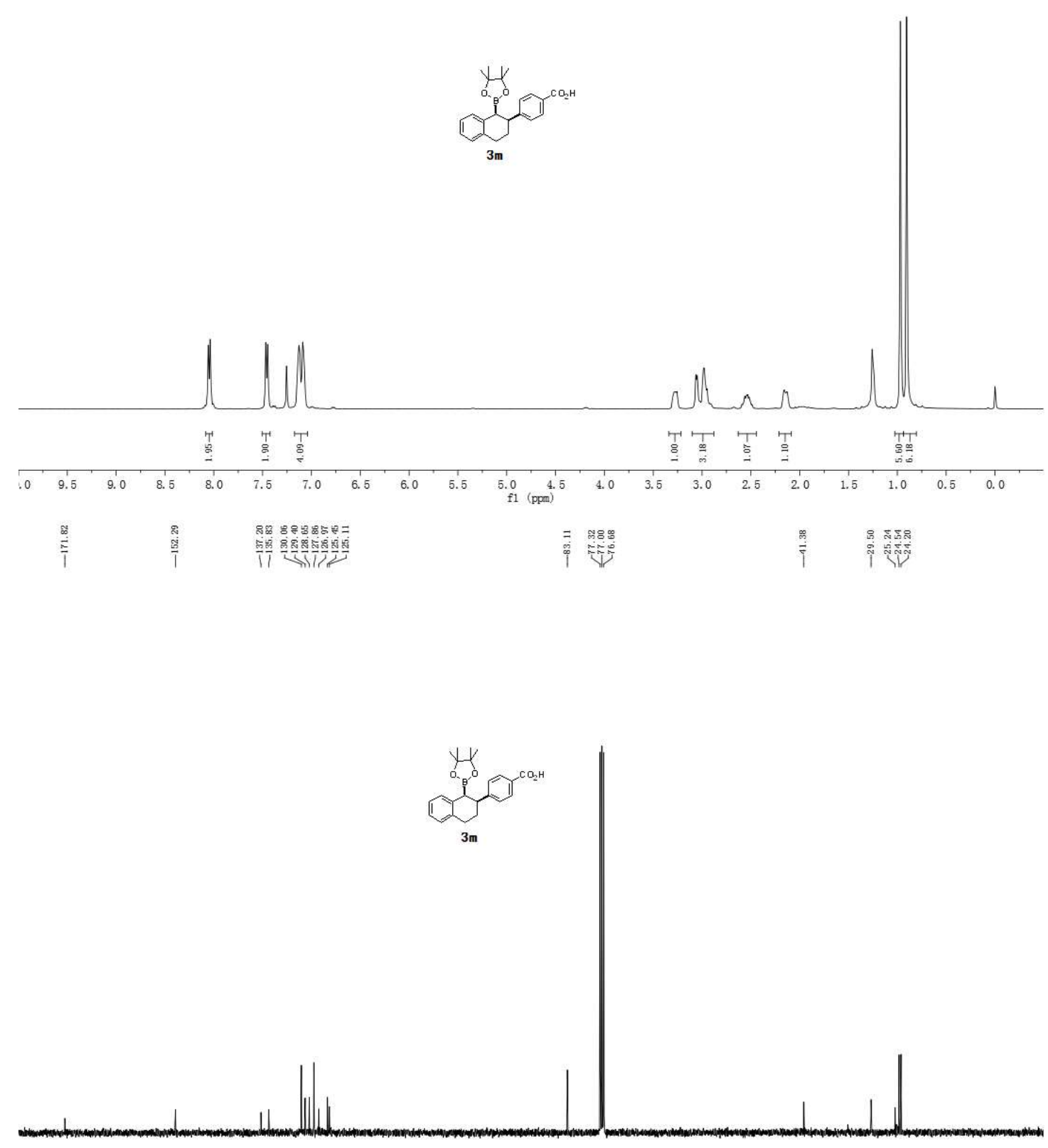

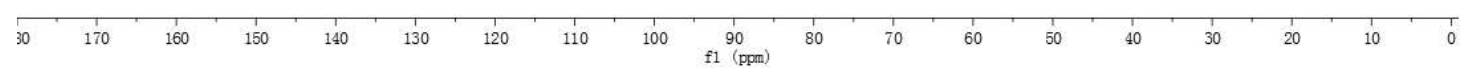



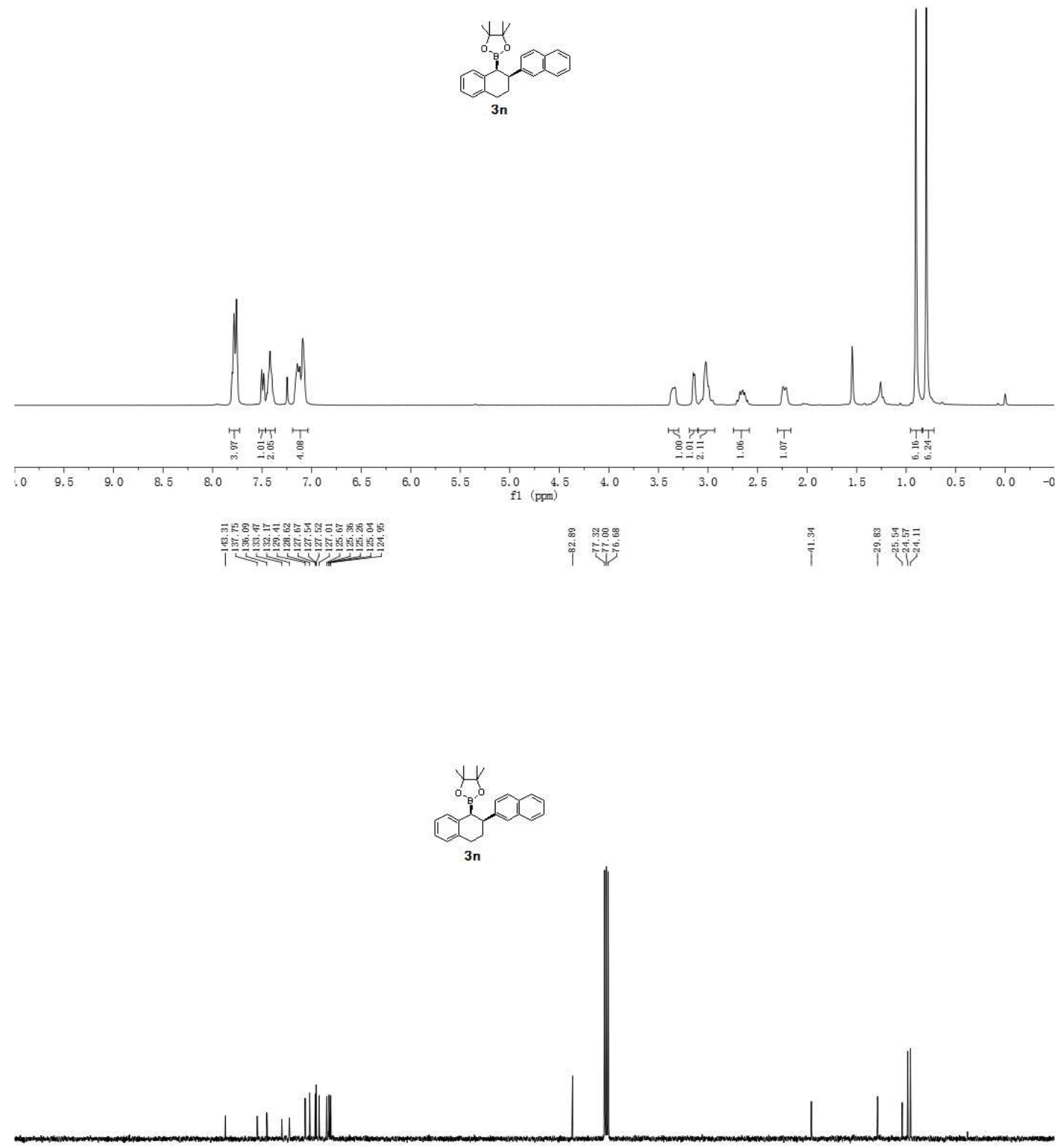

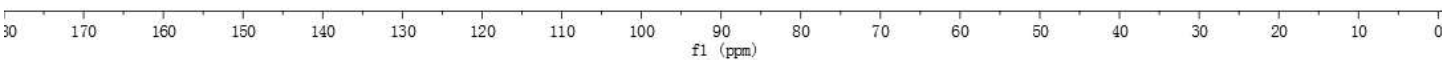



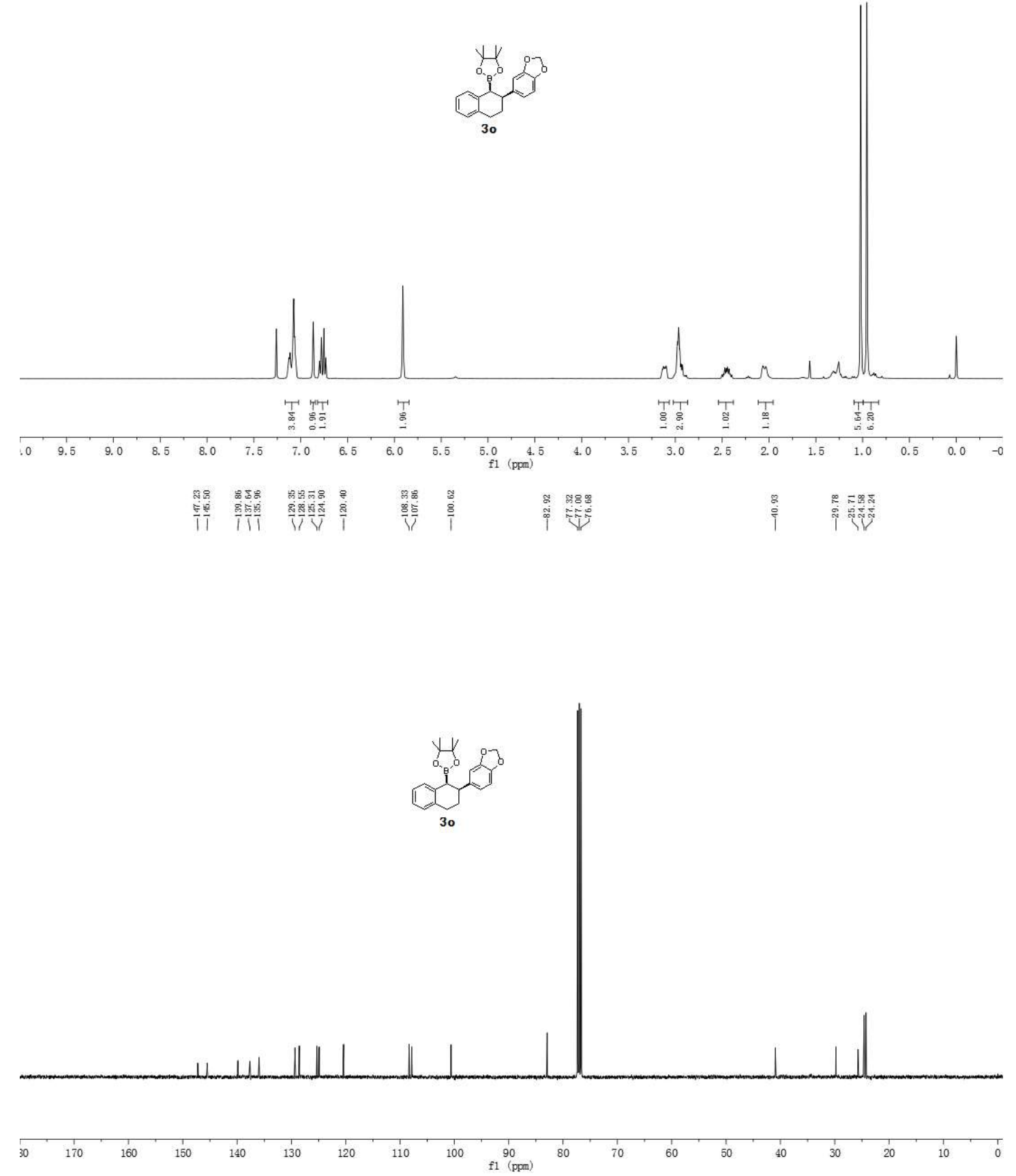

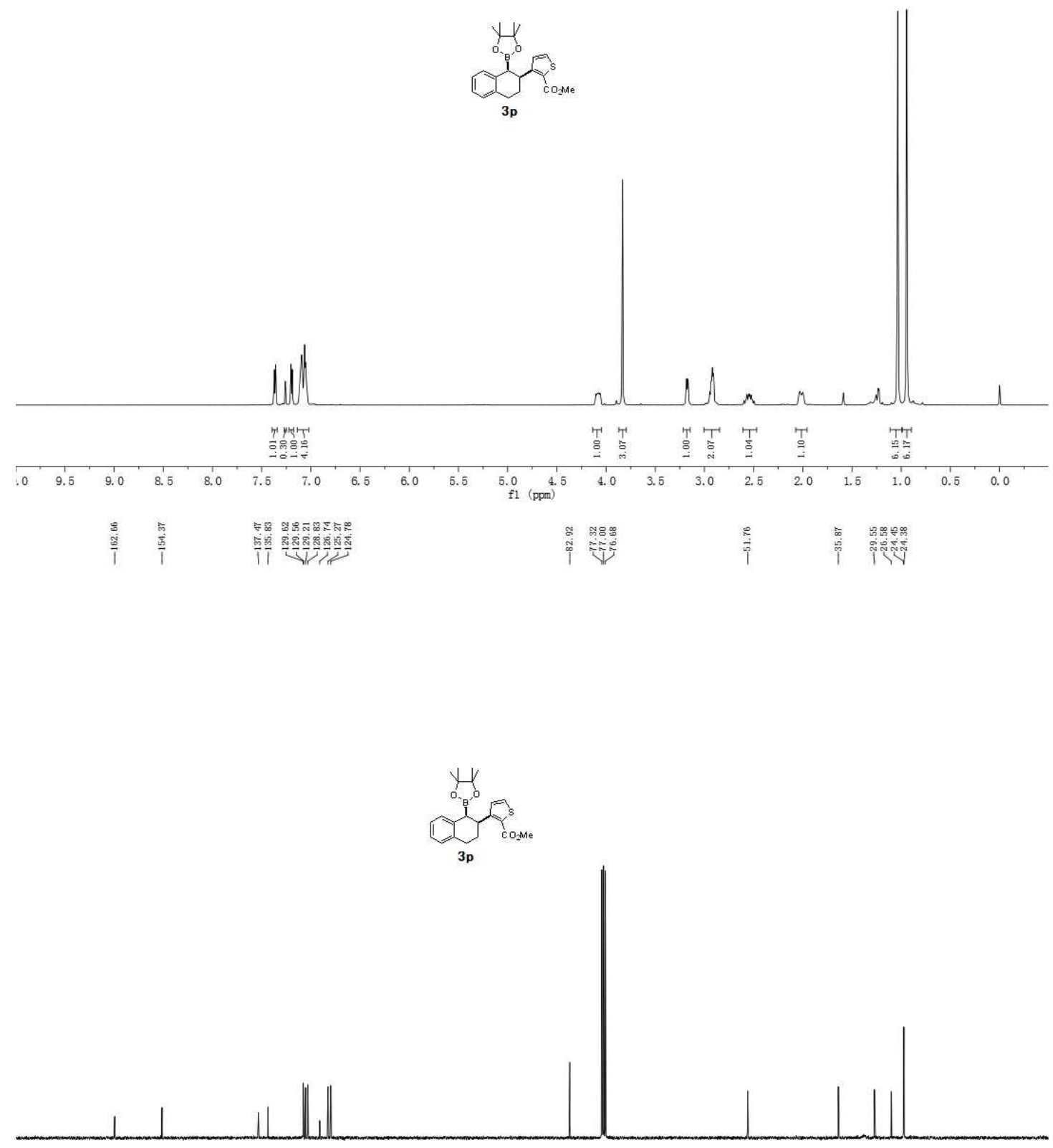

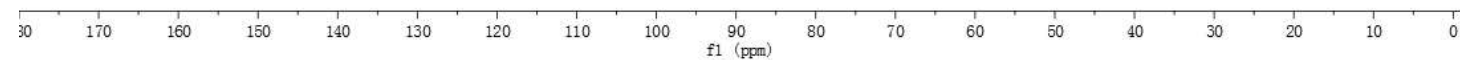




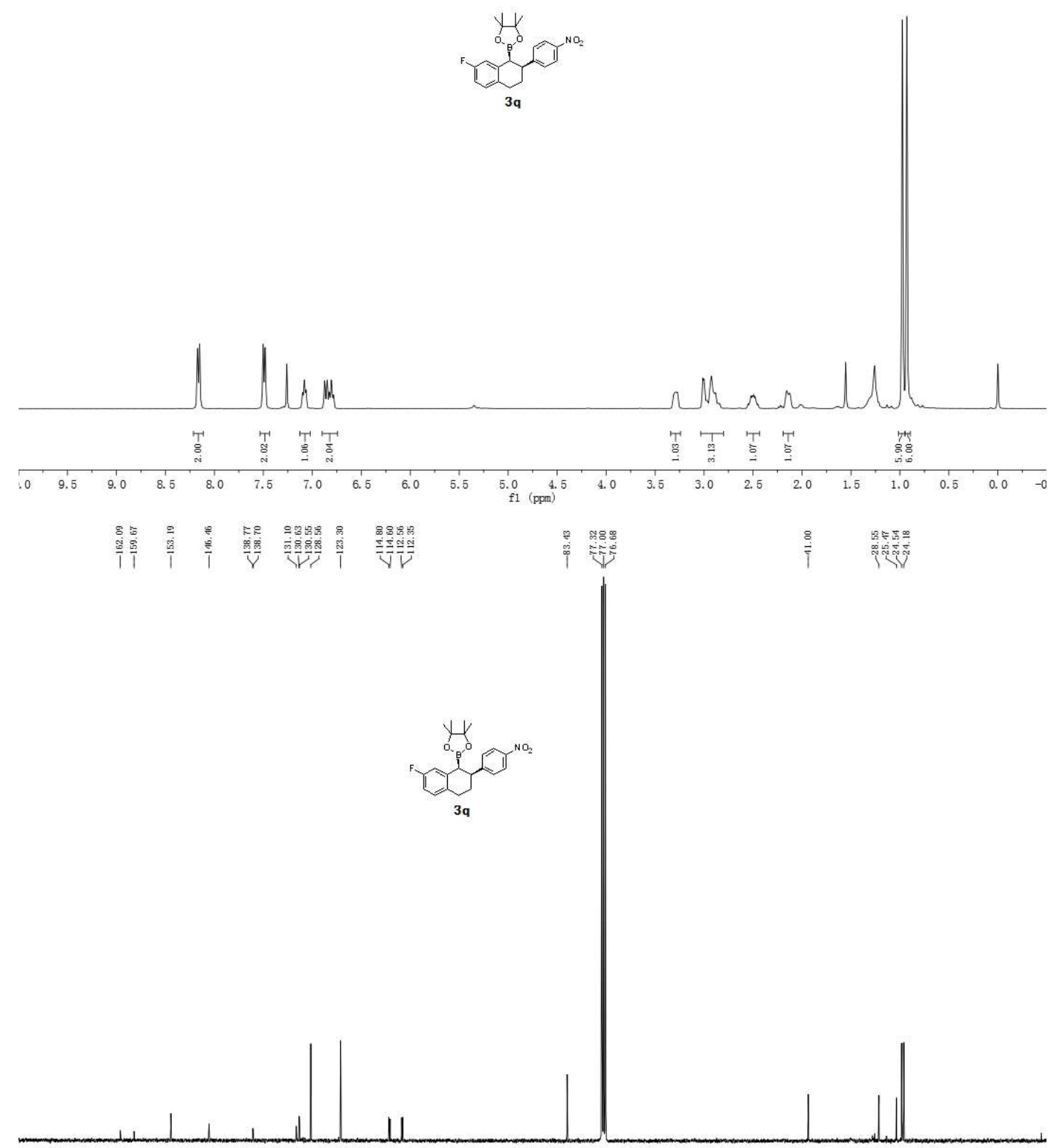

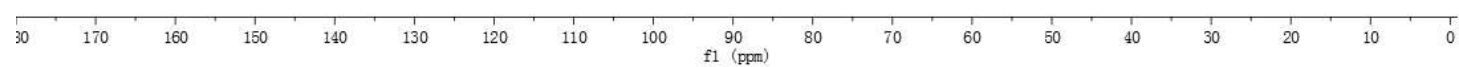




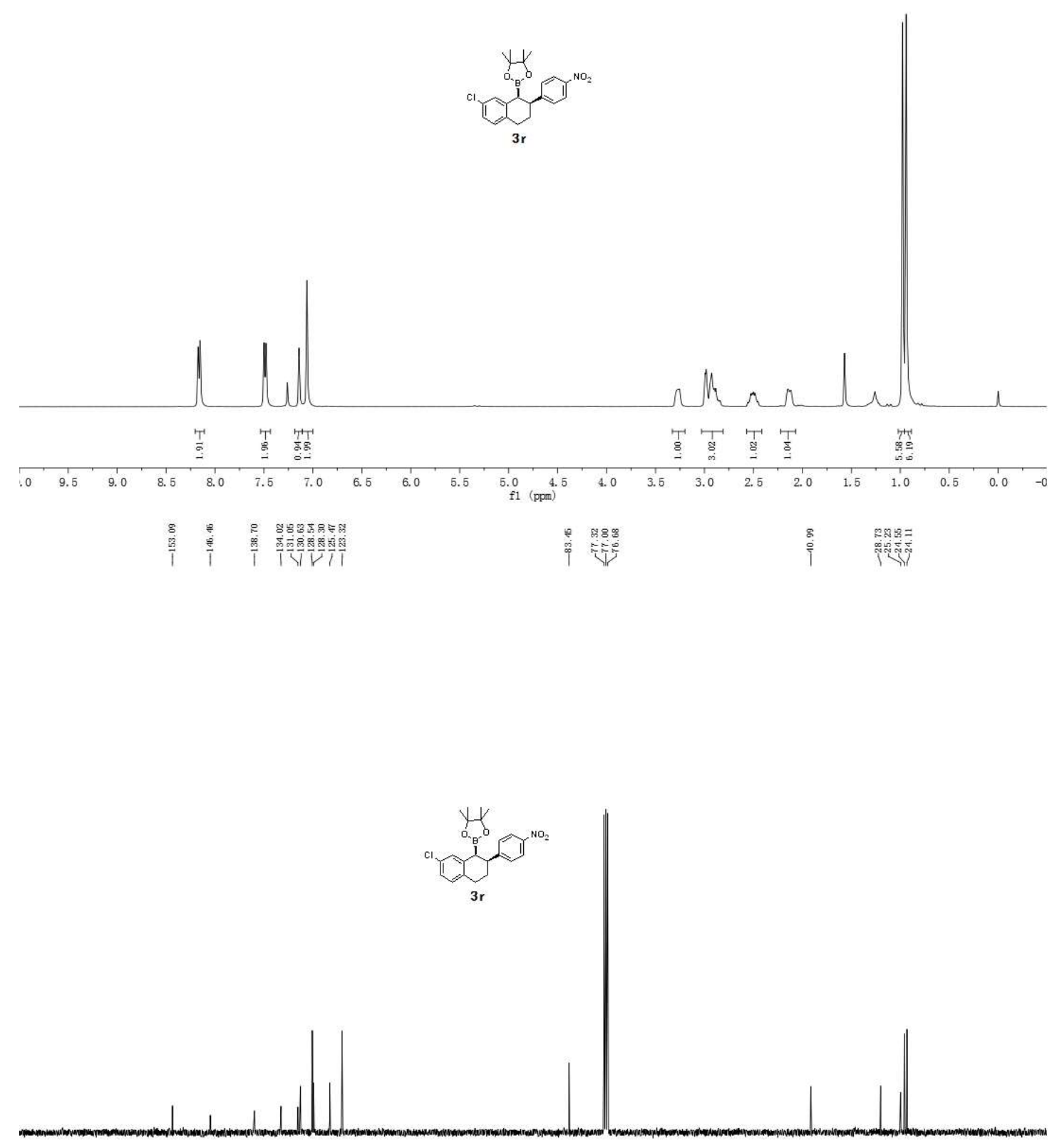

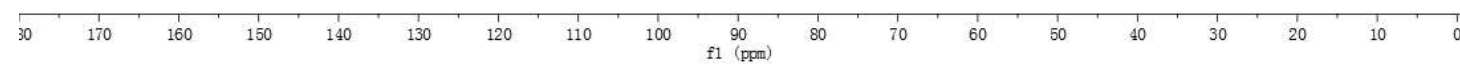




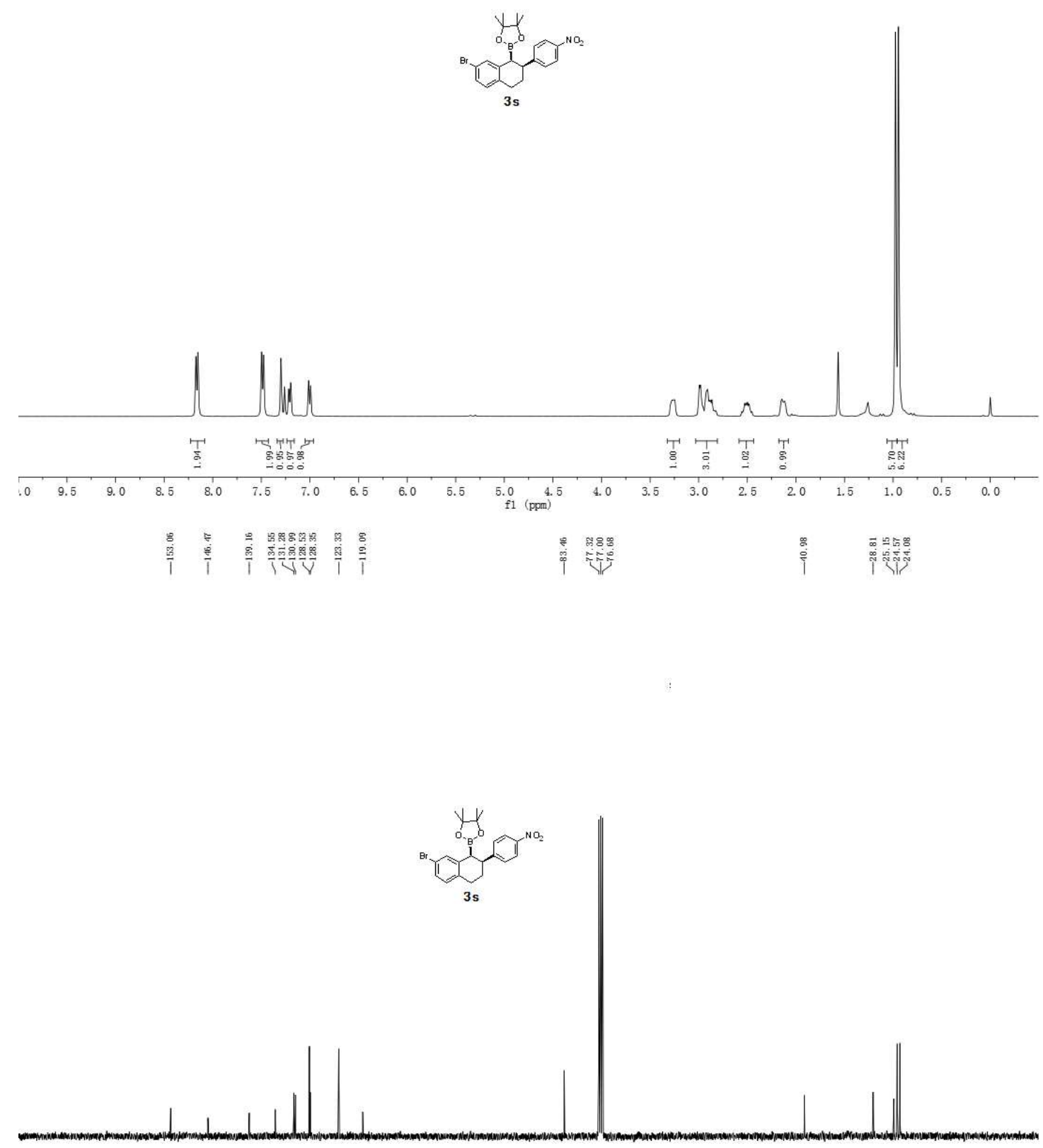

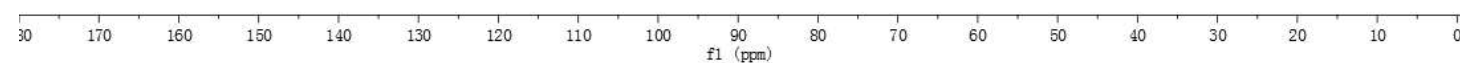



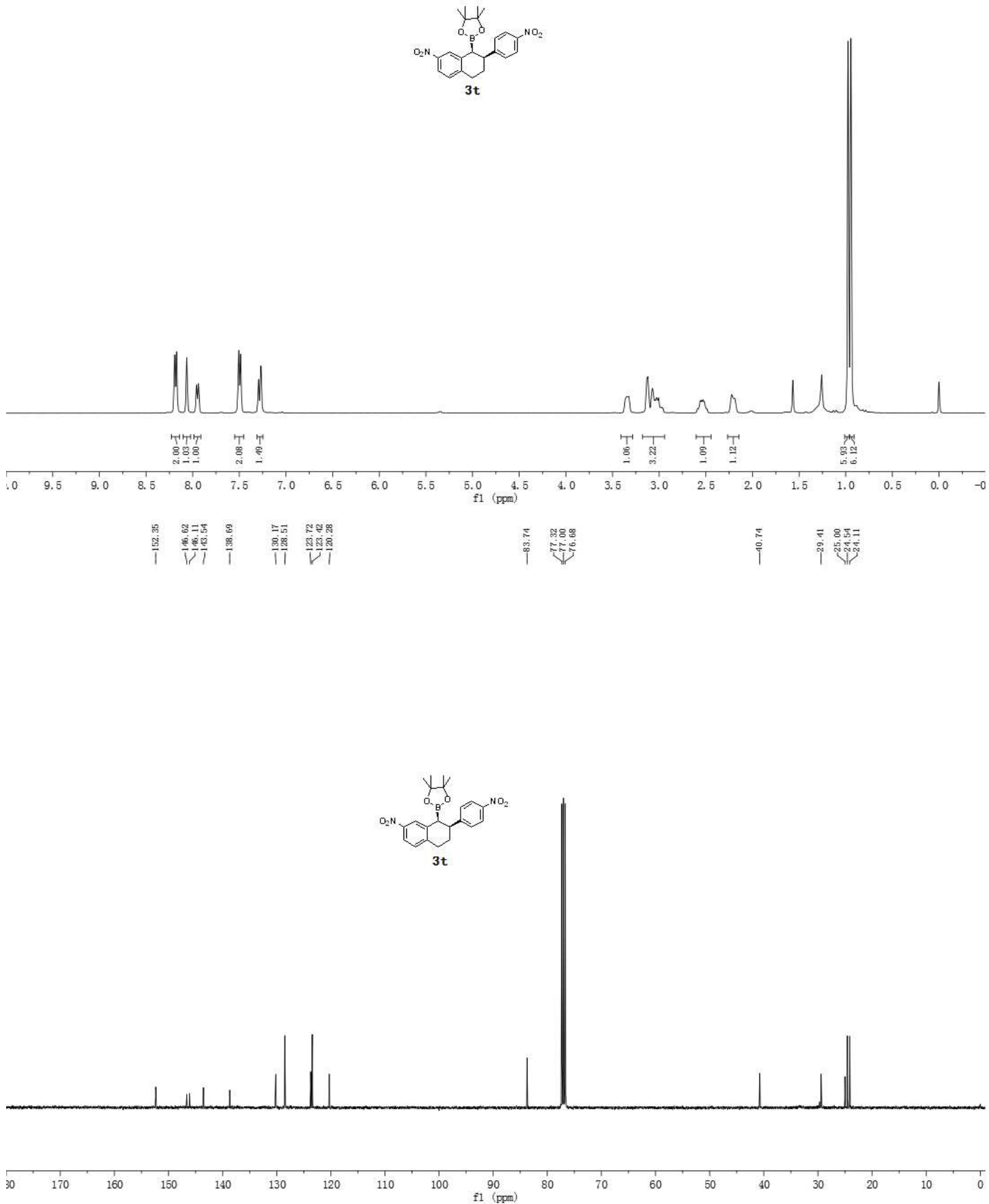

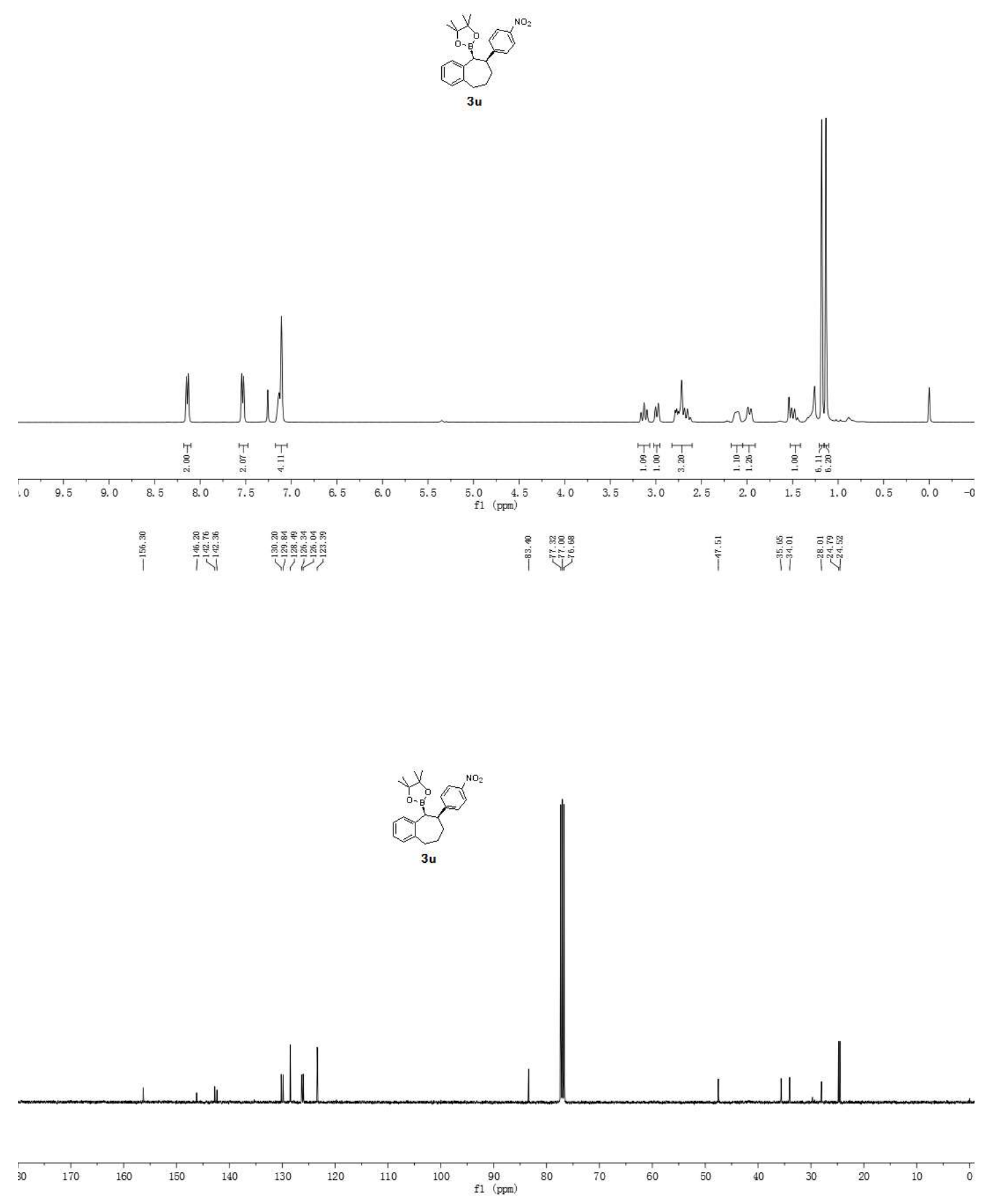

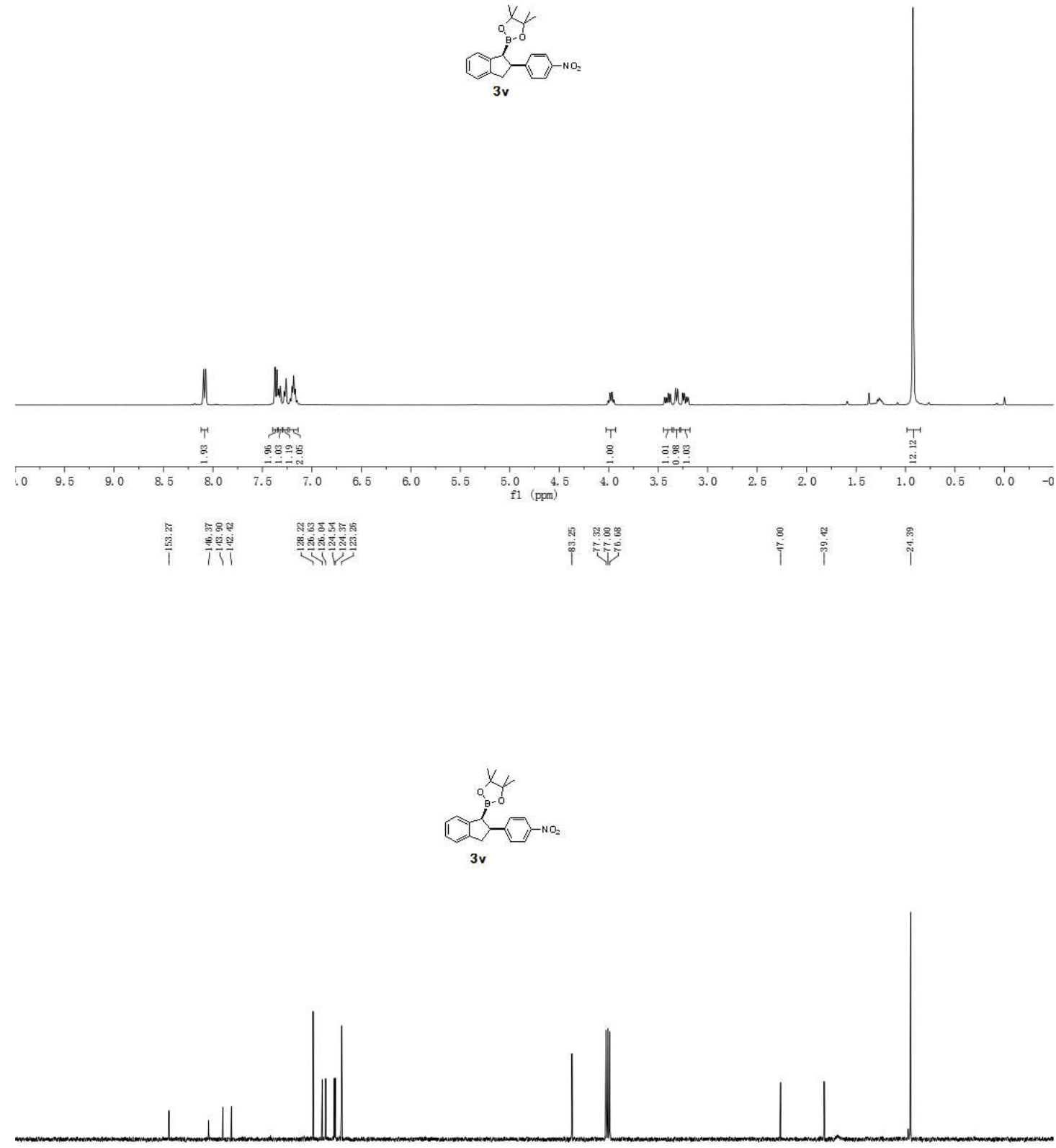

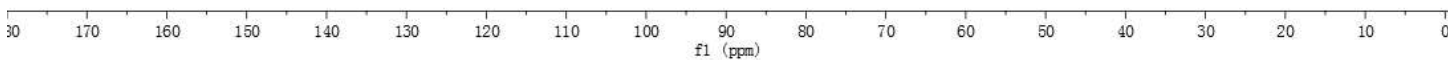



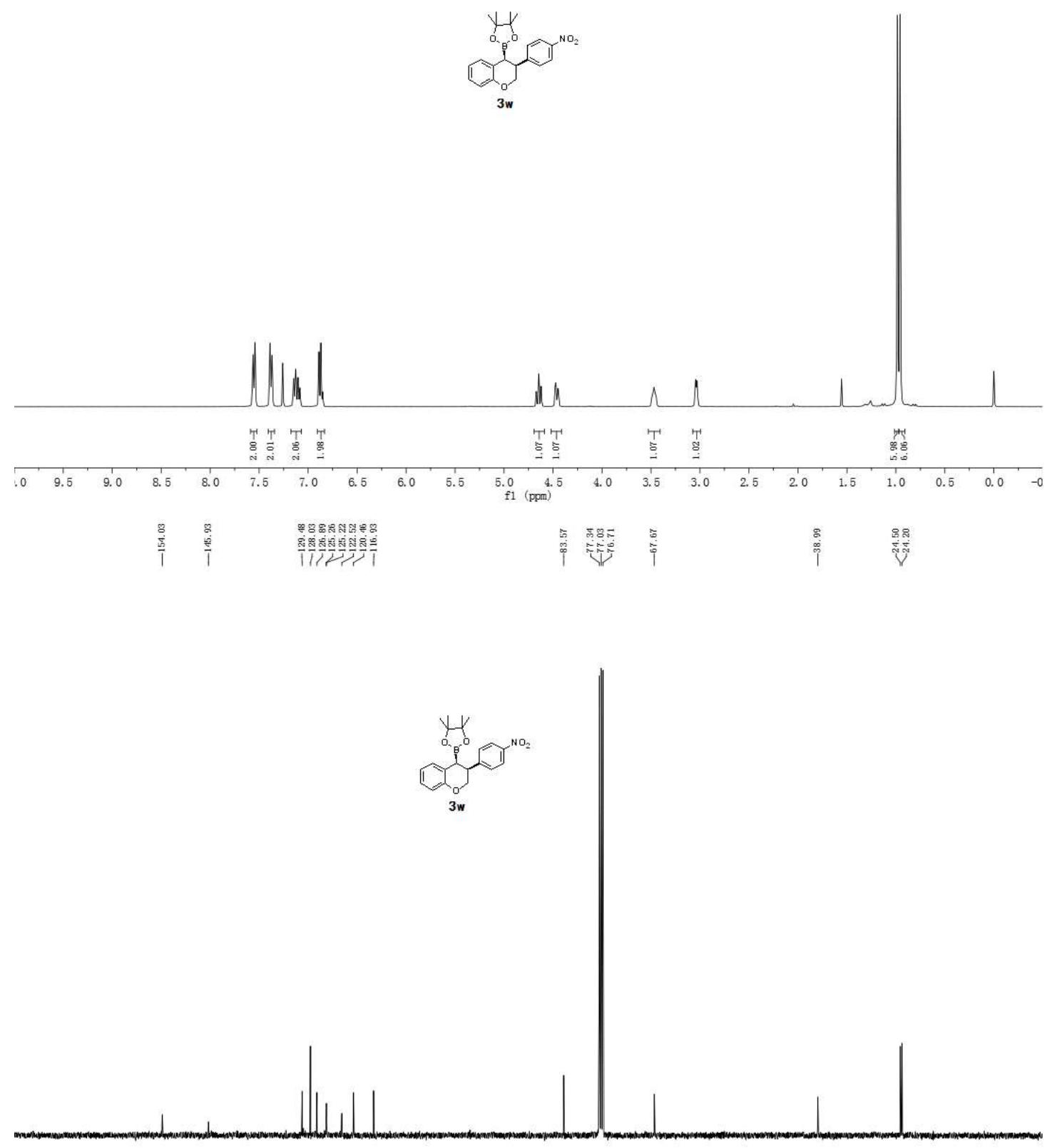

\begin{tabular}{lllllllllllllllll}
\hline 30 & 170 & 160 & 150 & 140 & 130 & 120 & 110 & 100 & 90 & 1 & 1 & 1 & 1 & 1 & 1 & 1 \\
(ppm) & 80 & 70 & 60 & 50 & 40 & 30 & 20 & 10 & 0
\end{tabular} 


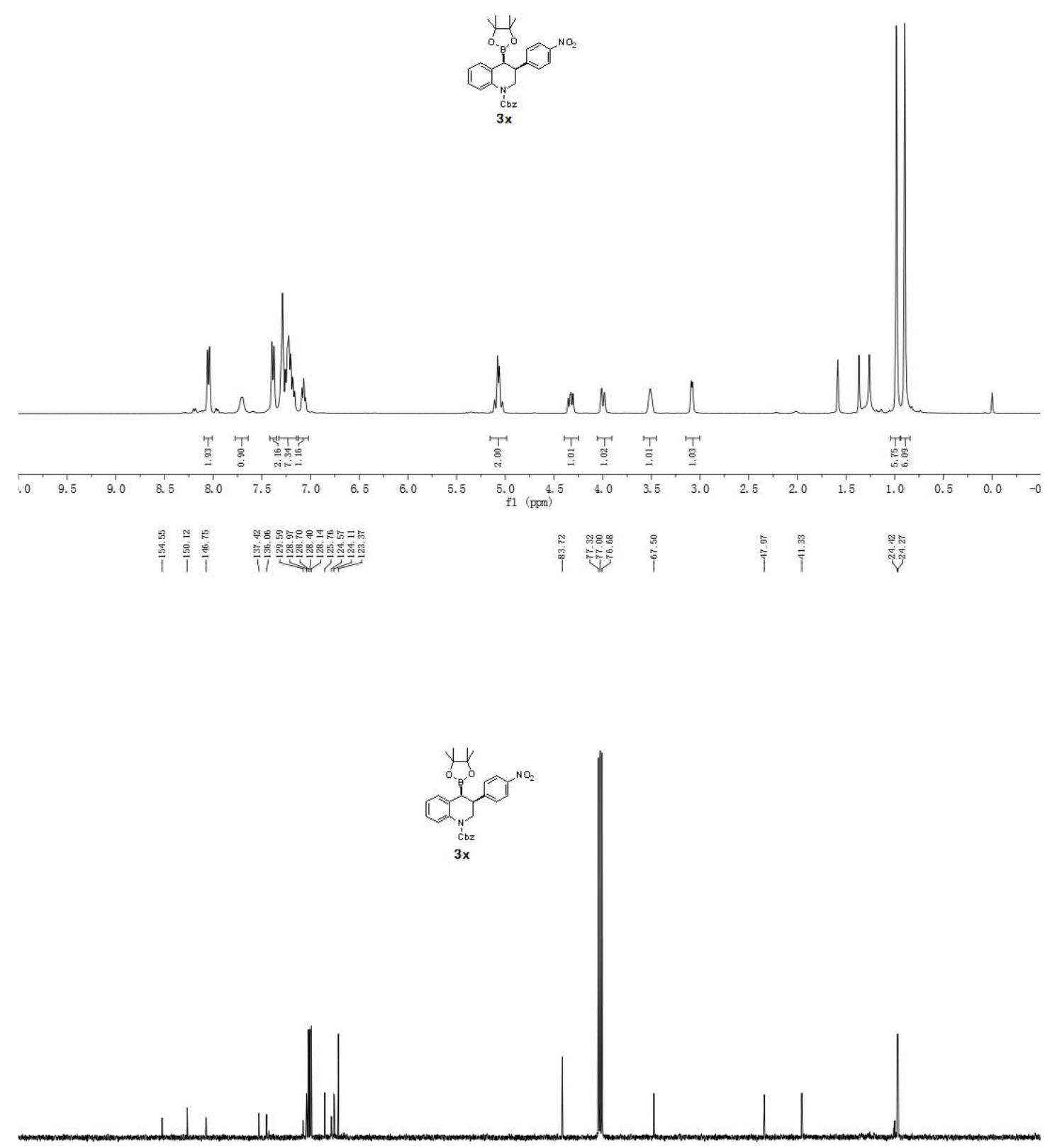

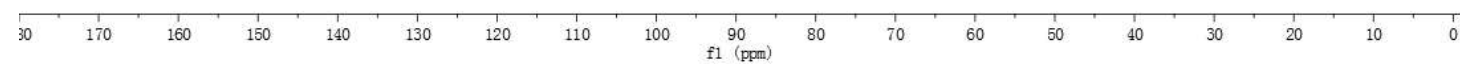




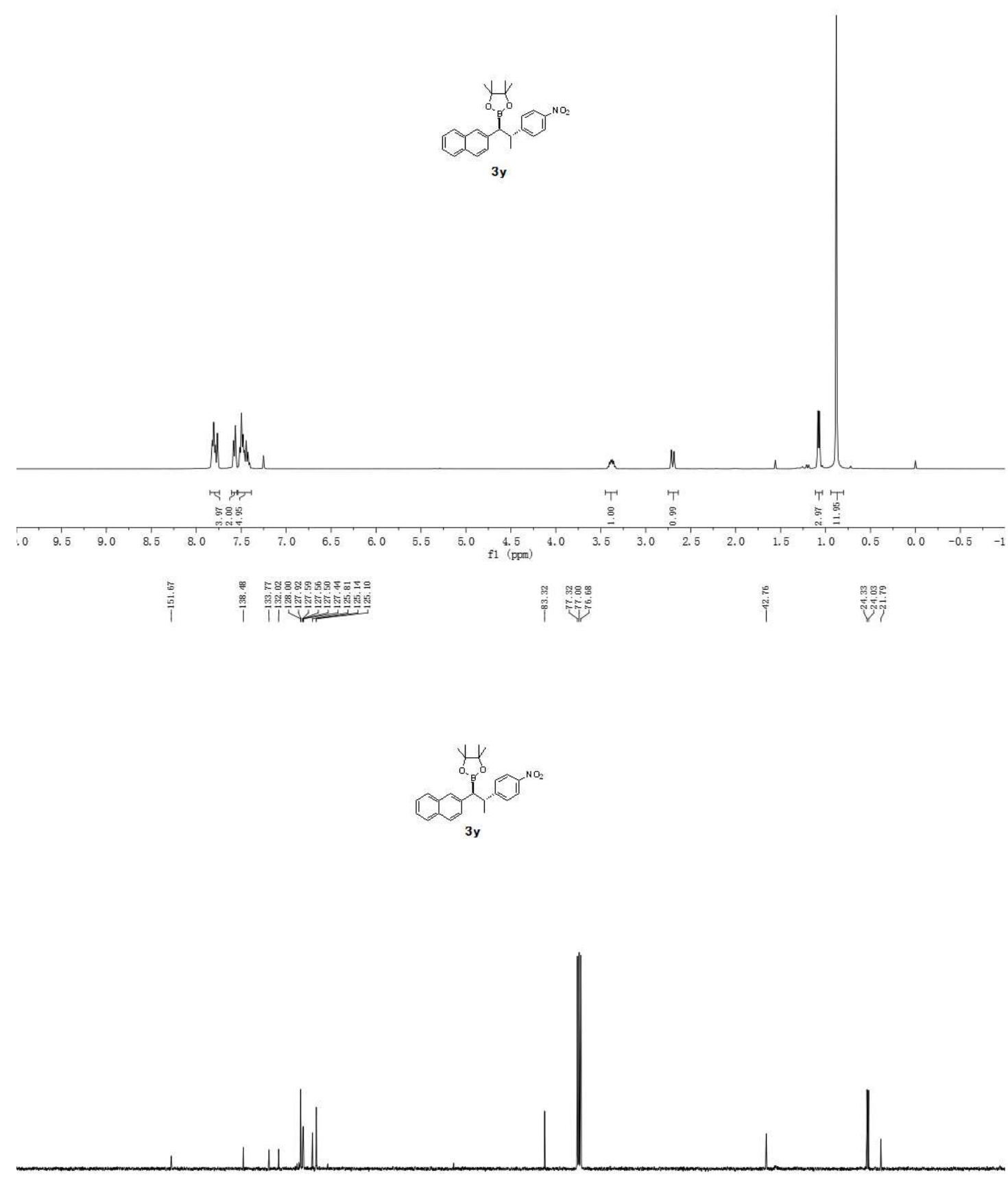

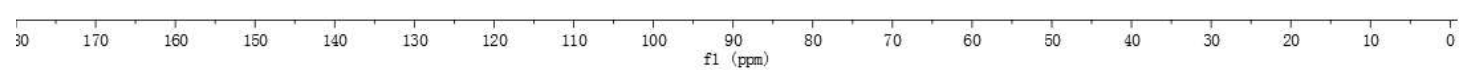



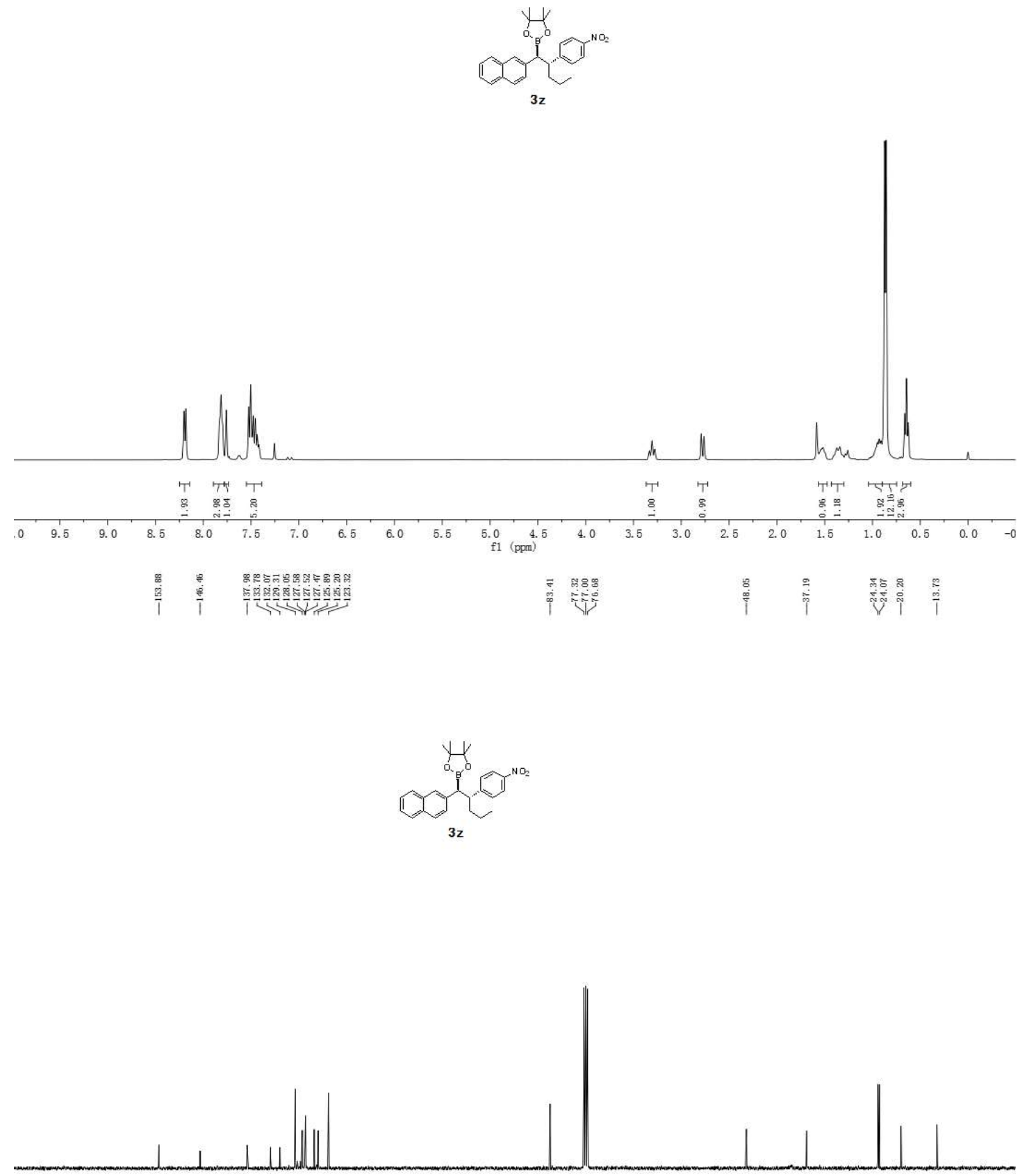

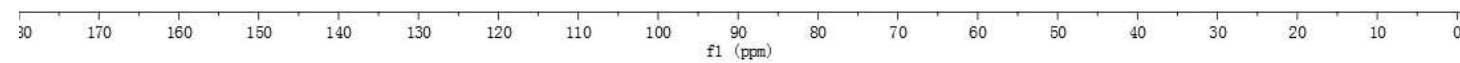



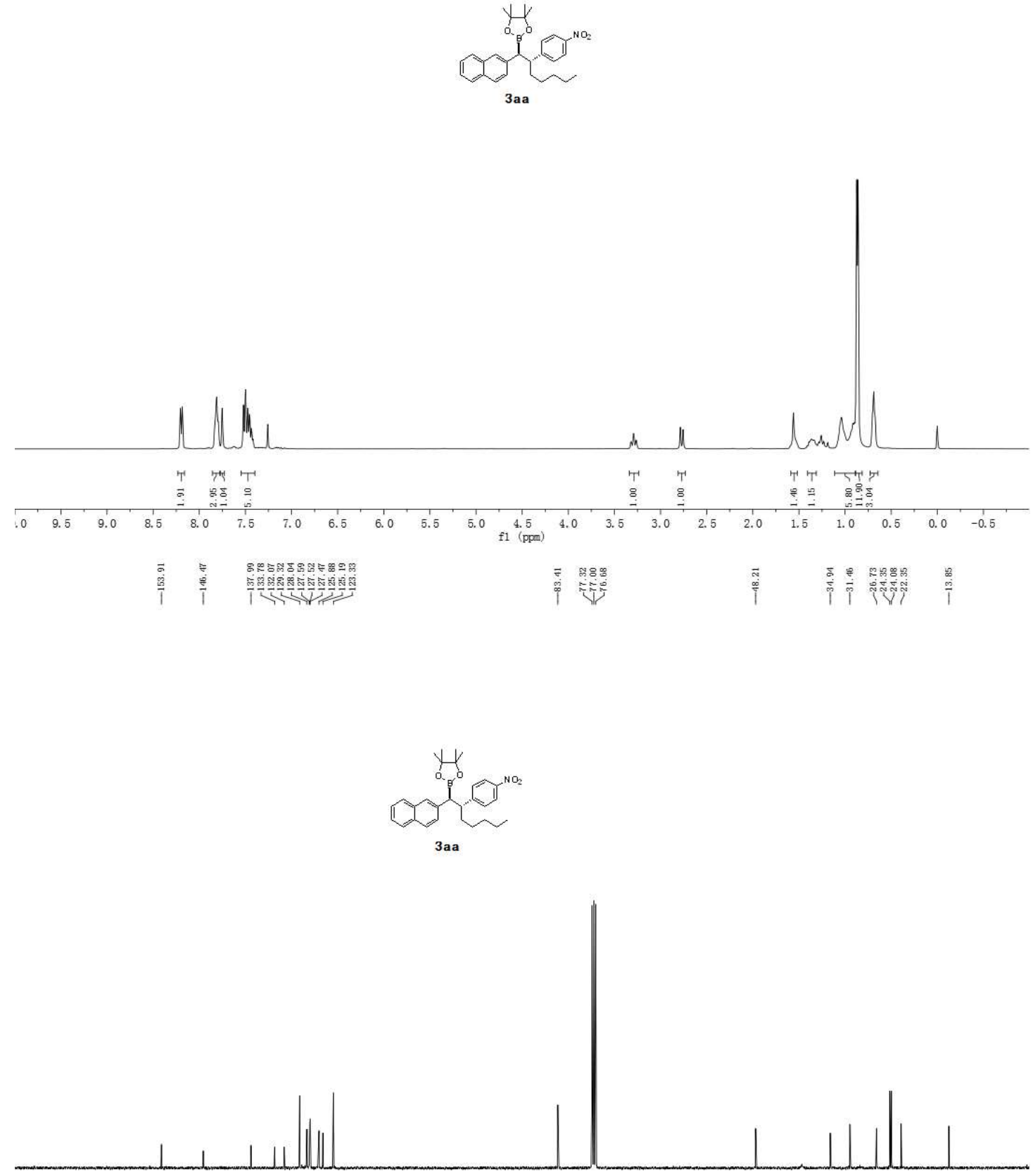

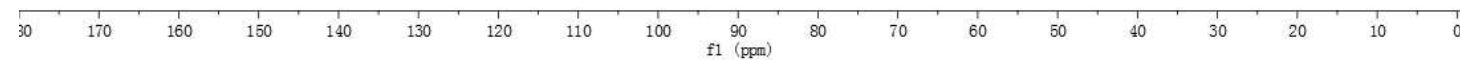




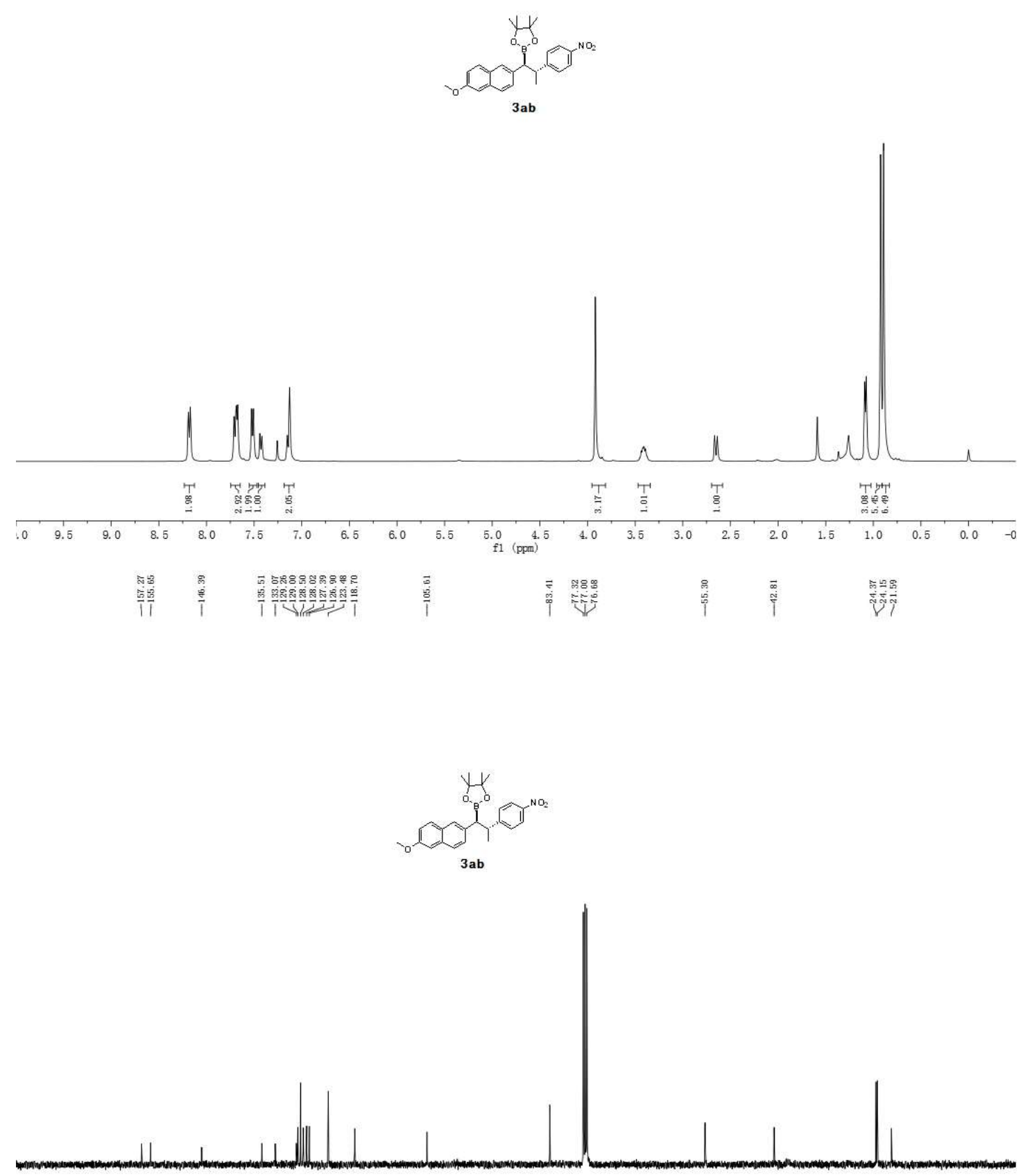

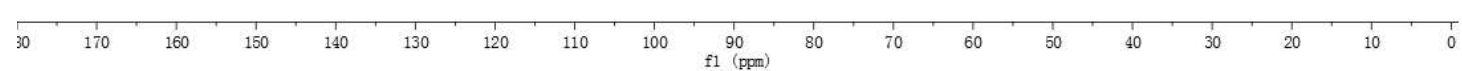




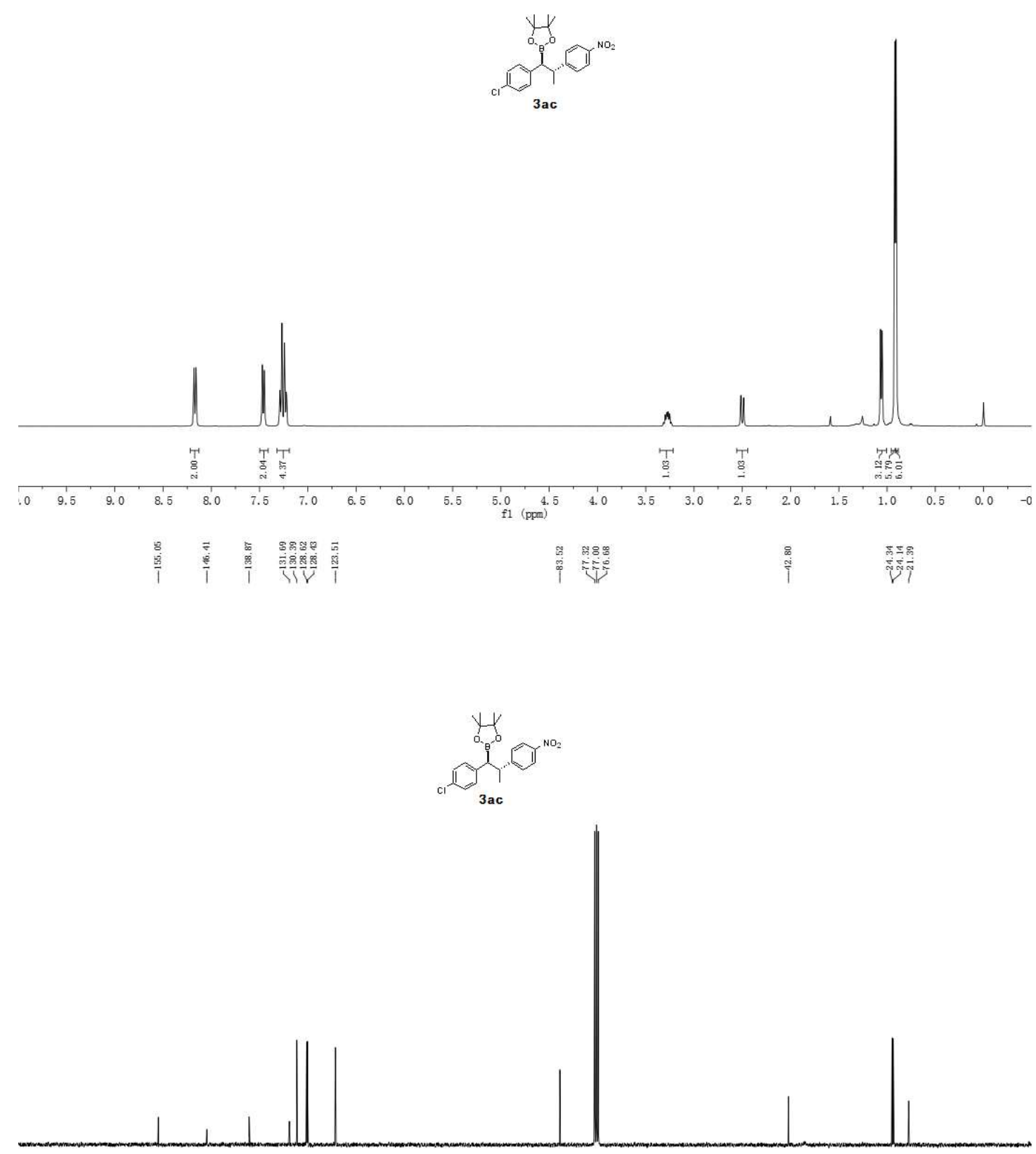

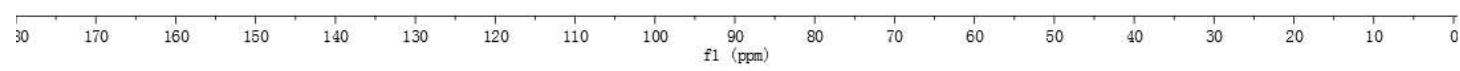



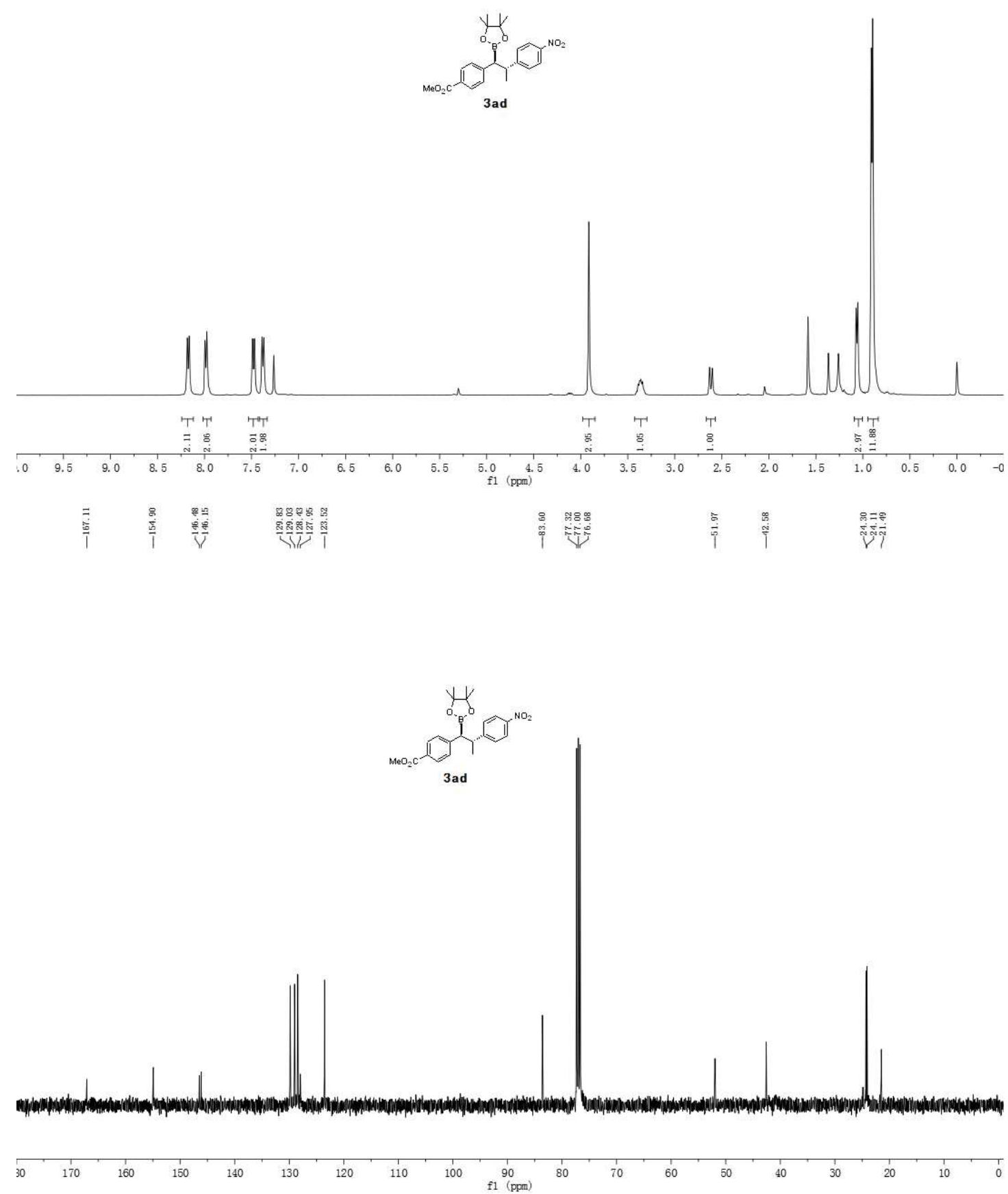

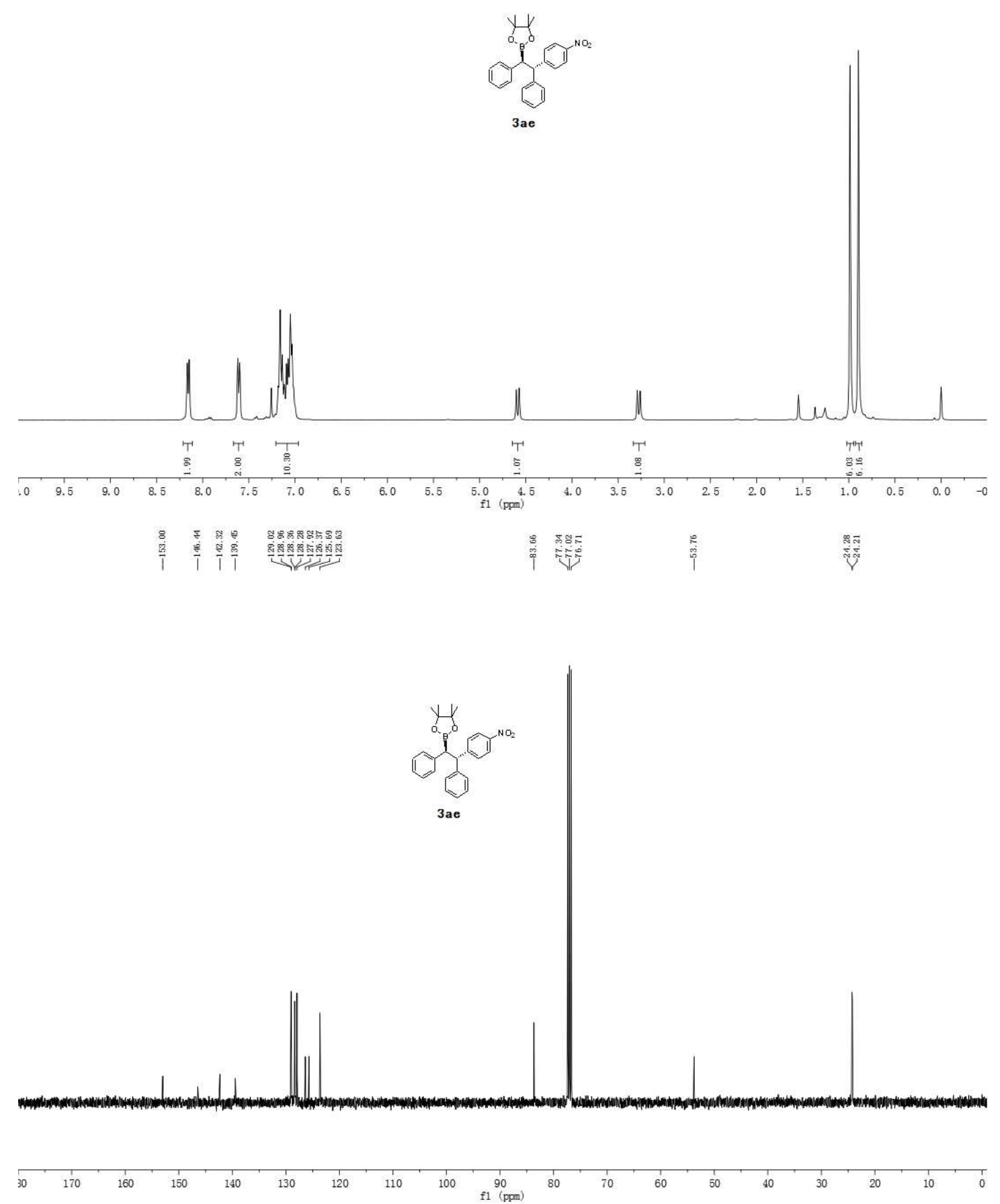

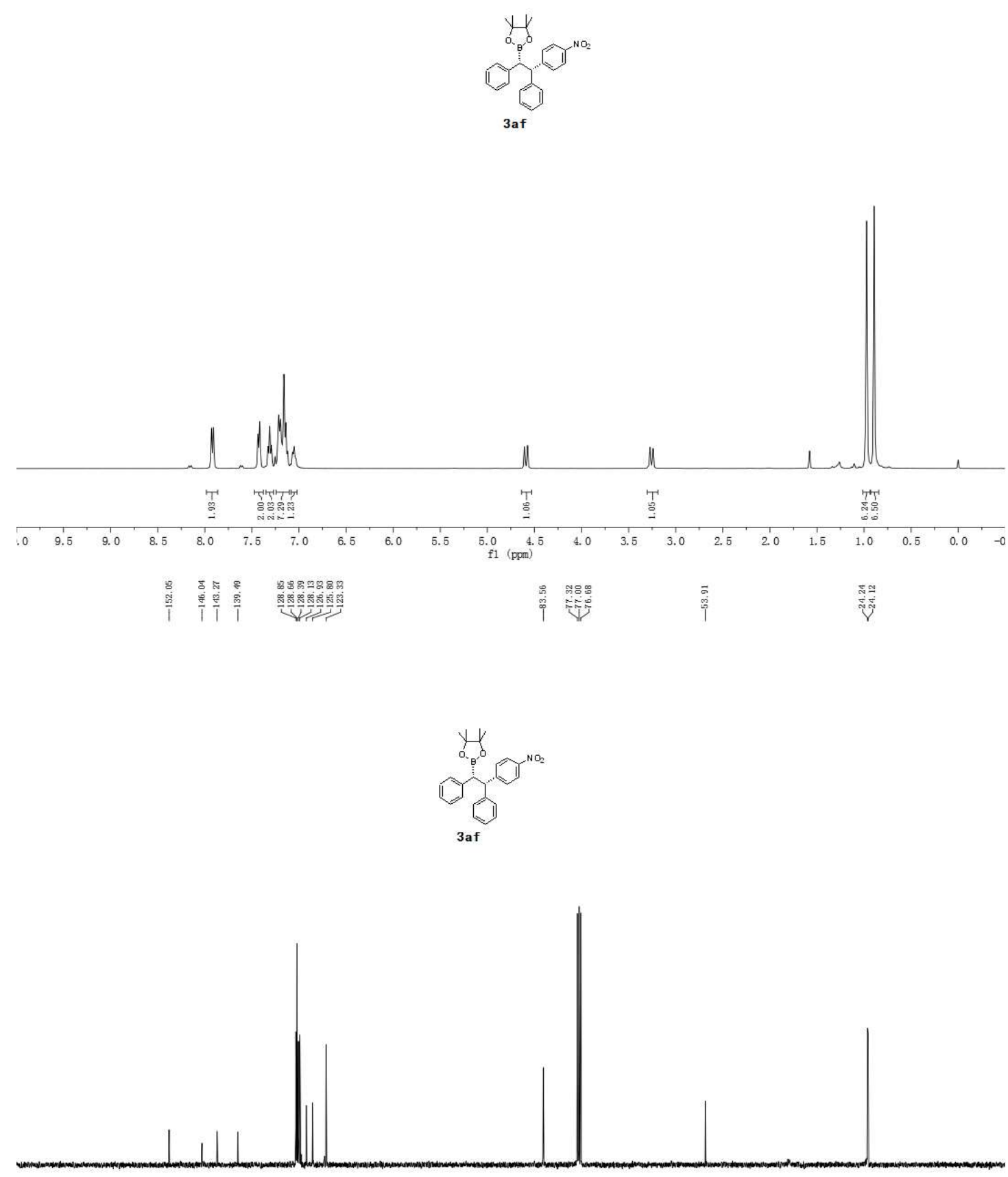

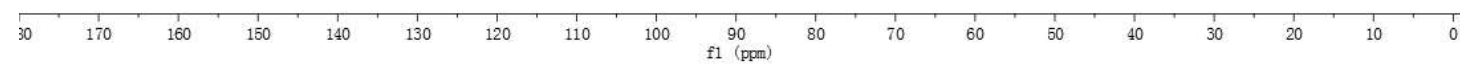


$\overbrace{3-\mathrm{H}}^{\mathrm{NO}}$
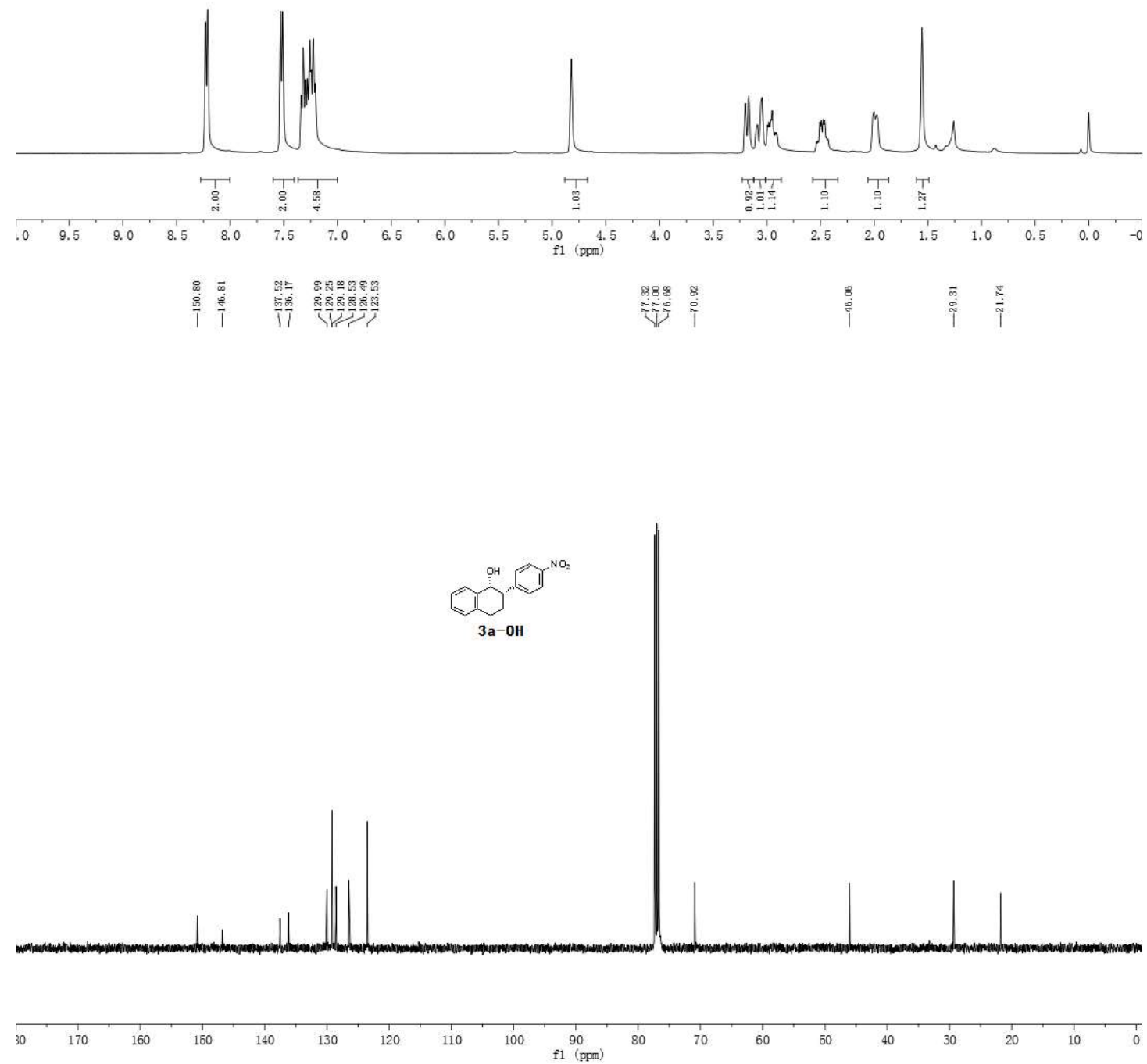

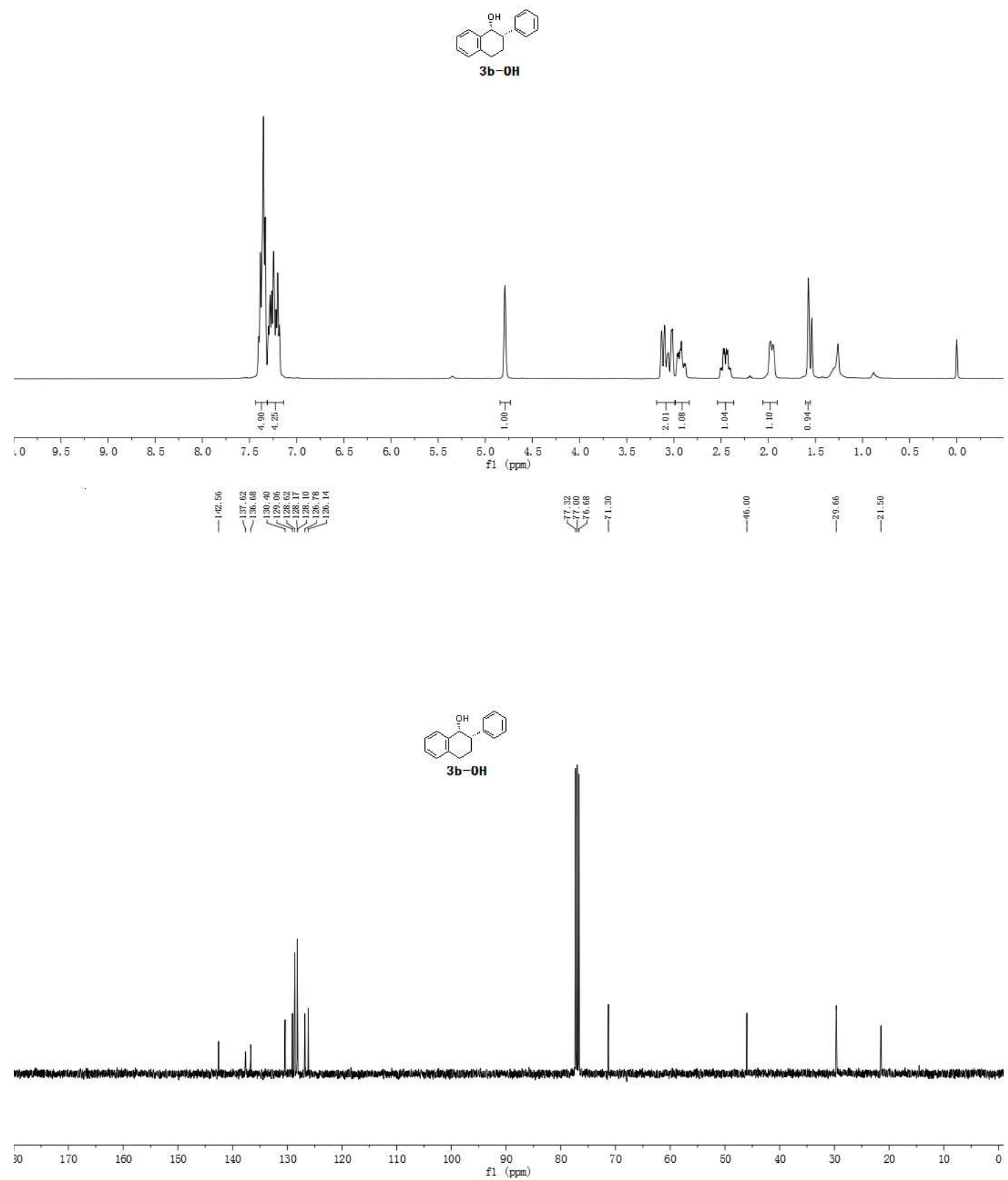

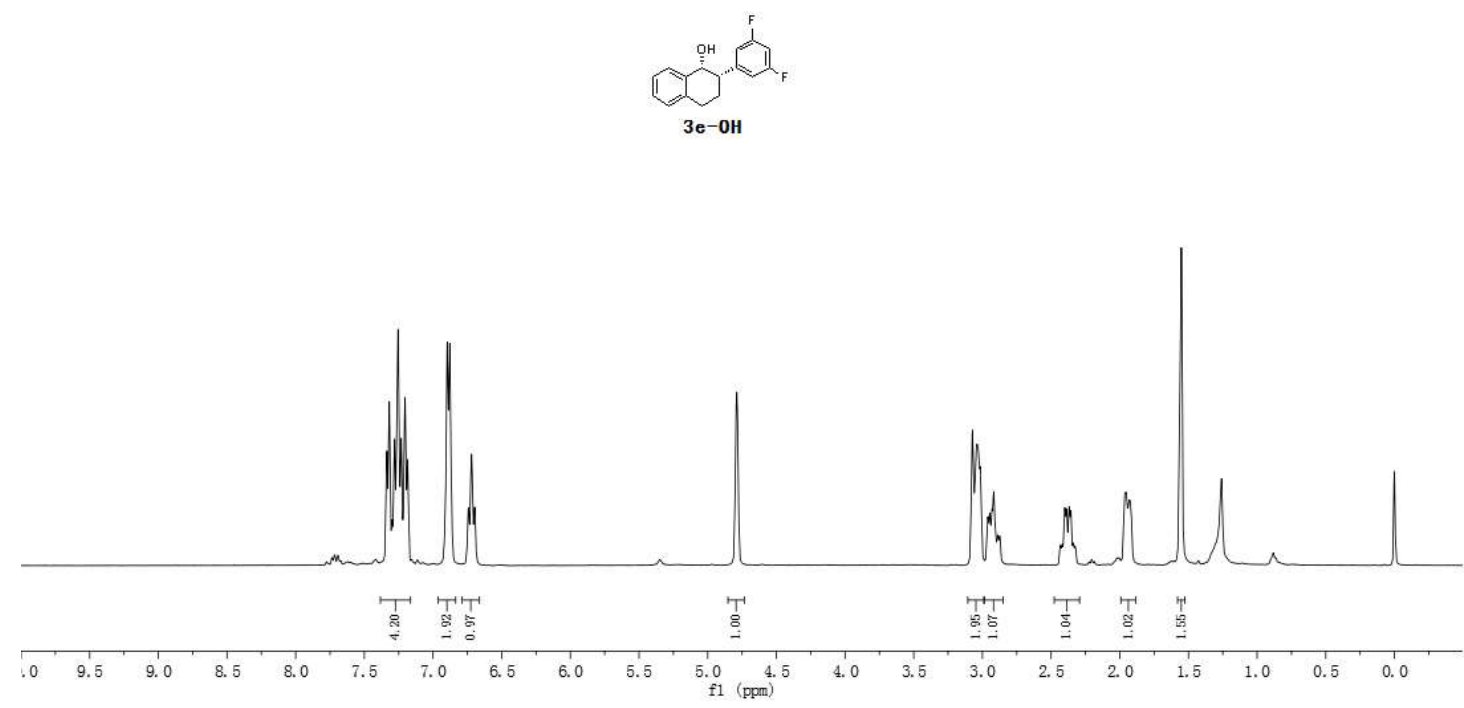

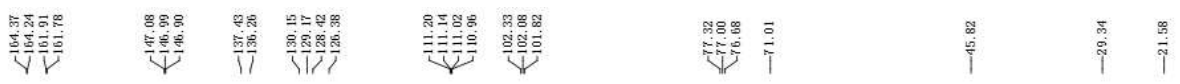

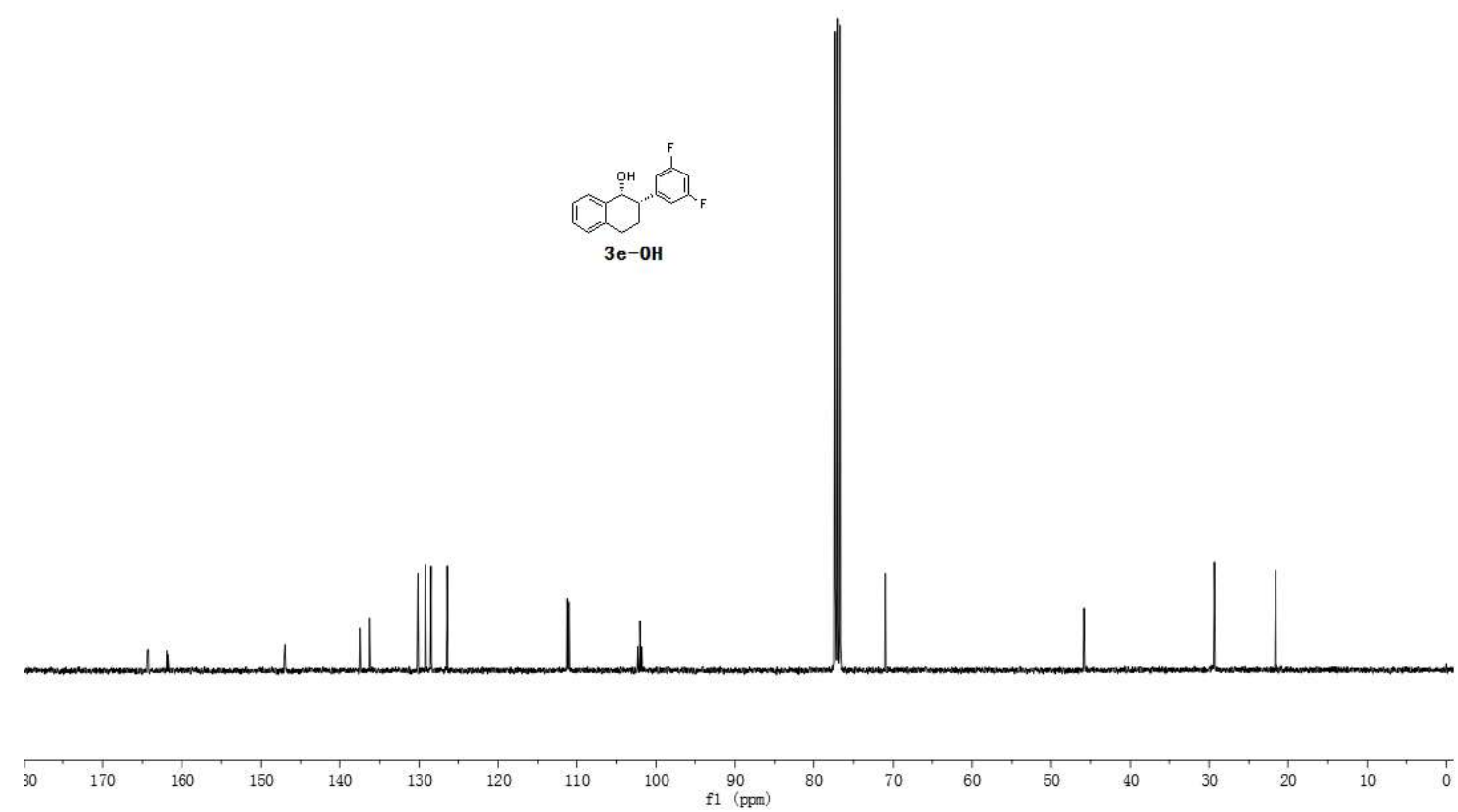



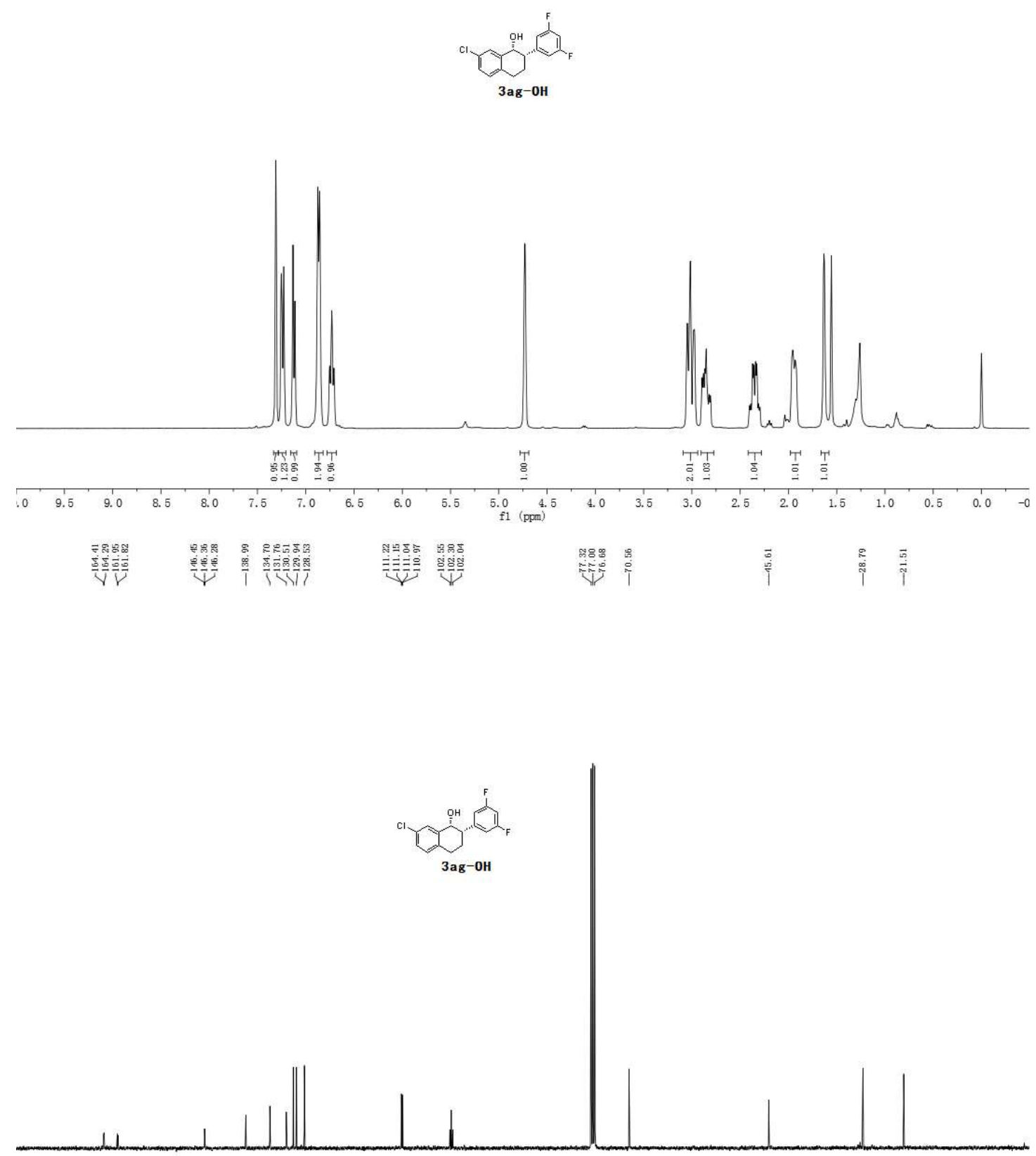

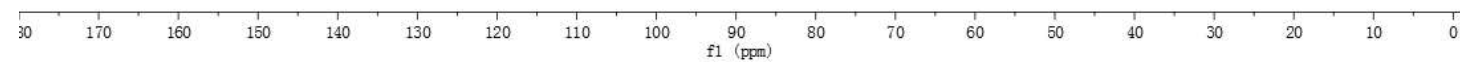



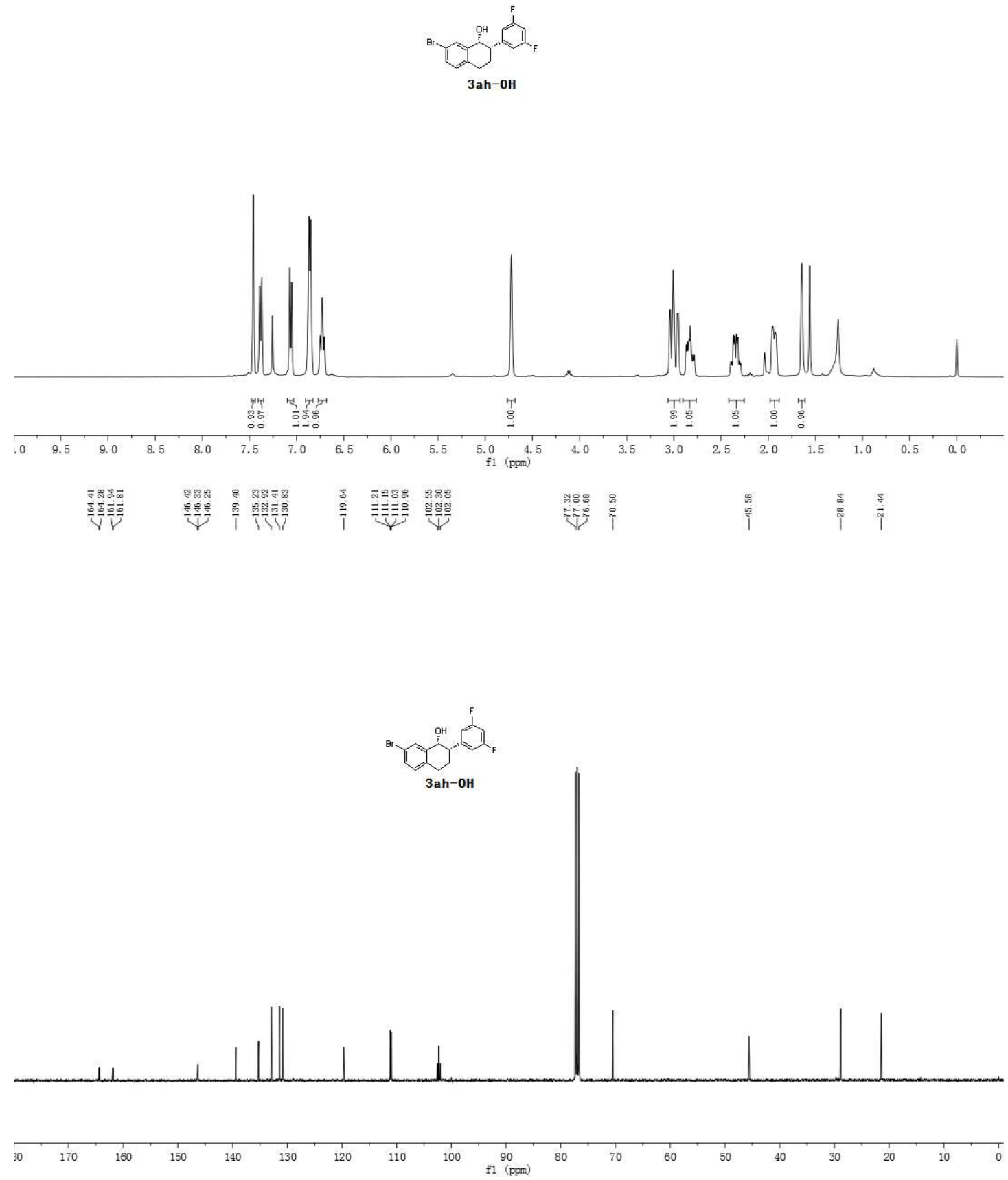

तT

โ 